\title{
Natural and Artificial Light-Harvesting Systems Utilizing the Functions of Carotenoids
}

Hideki Hashimoto ${ }^{\text {a, }}$, Yuko Sugai a, Chiasa Uragami a , Alastair T. Gardiner ${ }^{b}$ and Richard J. Cogdell ${ }^{\text {b }}$

${ }^{a}$ Department of Applied Chemistry for Environment, School of Science and Technology, Kwansei Gakuin University, 2-1 Gakuen, Sanda, Hyogo 669-1337, Japan

${ }^{\mathrm{b}}$ Institute of Molecular, Cell and Systems Biology, College of Medical Veterinary and Life Sciences, University of Glasgow, Avenue G12 8QQ, United Kingdom

* Corresponding author: e-mail, hideki-hassy@kwansei.ac.jp

\section{Abbreviations}

Chl, Chlorophyll; SeaWiFS, Sea-viewing Wide Field-of-view Sensor; ATP, adenosine triphosphate; NADPH, the reduced form of nicotine adenine dinucleotide phosphoric acid; RC, reaction centre; PSI, photosystem I; PSII, photosystem II; FMO, Fenna-Matthews-Olson; TEM, transmission electron microscopy; AFM, atomic force microscopy; Bchl, bacteriochlorophyll; FCP, fucoxanthin-Chl a/c protein; ICM, intra-cytoplasmic membranes; Rba., Rhodobacter; Rsp., Rhodospirillum; Rps., Rhodopseudomonas; Blc., Blastochloris; Bphe, bacteriopheophytin; EET, excitation energy transfer; ICT, intramolecular charge-transfer; TPE, two-photon excitation; FWM, four-wave mixing; SWM, six-wave mixing; CARS, Coherent Anti-Stokes Raman Scattering; ET, energy-transfer; IC, internal conversion; FSM, folded-sheet mesoporous material; C-P, carotenoid-porphyrin; C-P-Q, carotenoid-porphyrin-quinone; C-P-C60, carotenoid-porphyrin-fullerene 


\begin{abstract}
Carotenoids are essential pigments in natural photosynthesis. They absorb in the blue-green region of the solar spectrum and transfer the absorbed energy to (bacterio-)chlorophylls, and so expand the wavelength range of light that is able to drive photosynthesis. This process is an example of singlet-singlet energy transfer and so carotenoids serve to enhance the overall efficiency of photosynthetic light reactions. Carotenoids also act to protect photosynthetic organisms from the harmful effects of excess exposure to light. In this case, triplet-triplet energy transfer from (bacterio-)chlorophyll to carotenoid plays a key role in this photoprotective reaction. In the light-harvesting pigment-protein complexes from purple photosynthetic bacteria and chlorophytes, carotenoids have an additional role, namely the structural stabilization of those complexes. In this article we review what is currently known about how carotenoids discharge these functions. The molecular architecture of photosynthetic systems will be outlined to provide a basis from which to describe the photochemistry of carotenoids, which underlies most of their important functions in photosynthesis. Then, the possibility to utilize the functions of carotenoids in artificial photosynthetic light-harvesting systems will be discussed. Some examples of the model systems are introduced.
\end{abstract}




\section{Introduction}

\subsection{Carotenoids in Photosynthesis}

Most carotenoids found on earth are synthesized by photosynthetic organisms [1]. The scale of the manufacture of carotenoids is enormous. The total amount of chlorophyll (Chl), including all sub-types and bacteriochlorophyll, synthesized each year has been determined using data from the SeaWiFS (Sea-viewing Wide Field-of-view Sensor) satellite. Each year it has been estimated that about one billion tons of chlorophyll is produced [2]. Assuming that the production of carotenoids is about one-tenth of that of chlorophyll, then the annual amount of carotenoid produced by photosynthetic organisms can be estimated to be about 1000 million tons.

Chls that play major role in photosynthesis do not absorb much light in the 450-550 $\mathrm{nm}$ region where the solar radiation profile (spectrum) at the surface of the Earth has its maximum intensity, however, this is precisely the region where carotenoids absorb light strongly. They are able to transfer this excitation energy to the Chls, thereby making it available to power photosynthesis [3, 4]. This energy-transfer reaction allows carotenoids to function as accessory light-harvesting pigments, broadening the spectral range over which light can support photosynthesis. This light-harvesting role of carotenoids is particularly significant in the cases of purple photosynthetic bacteria, heterokontophyta (e.g., diatoms and brown algae) and dinoflagellates all of which tend to occupy environmental niches where light intensity is usually limiting for growth and where, especially in the case of dinoflagellates, most of the available solar energy is in the $450-550 \mathrm{~nm}$ region [3]. However, the reason that carotenoids are essential for photosynthesis is not because of this light-harvesting role, rather because of their ability to prevent photo-damage that occurs under conditions of too much light $[3,5-7]$. The functions of carotenoids in photosynthesis can only really be understood in the context of the structure of the pigment-protein complexes in which most of them are organized. In the next section a brief account of these structures is provided.

\subsection{From Natural to Artificial Photosynthesis}

Figure 1 shows a schematic drawing of the light energy conversion processes 
carried out in photosynthetic membranes of oxygenic phototrophs, such as plants [8]. Plant photosynthesis is a complicated series of redox reactions, using light energy from the sun and electrons donated from the splitting of water, that produce bioenergy (ATP adenosine triphosphate) and reducing equivalents (NADPH - the reduced form of nicotine adenine dinucleotide phosphoric acid), which are subsequently consumed in the reduction of carbon dioxide to produce a fuel (carbohydrates). During these processes molecular oxygen is produced as a final by-product. In other words, photosynthesis produces all the food that is eaten and is the origin of all food chains. It also produces all the oxygen that we breathe. Therefore, photosynthesis is ultimately the source of all life inhabiting the Earth.

Light harvesting pigment-protein complexes (also called photosynthetic antenna proteins) absorb light energy from the sun to initiate the above-mentioned photosynthetic reactions. Thus, harvested solar energy is transferred in the form of excitation energy to one of two reaction centre (RC) complexes, either Photosystem II (PSII) or Photosystem I (PSI). In these RC complexes, an electric charge separation reaction takes place through the subsequent redox reactions and electromotive force is accumulated. The synthesis of ATP and NADPH are dependent upon this electromotive force. PSII holds an oxidation catalyst and PSI holds the reduction catalyst. PSII has the quinone type $\mathrm{RC}$ that is similar to purple photosynthetic bacteria and PSI contains the iron-sulfur cluster type RC that is similar to green sulfur bacteria. A remarkable feature of PSII is that the positive 'hole', produced on the $\mathrm{P}_{680}{ }^{+} \mathrm{Chl}$ dimer as a result of the loss of an electron during electric charge separation, is filled by the oxidative splitting of water molecules. This reaction is unique in biology and is performed by a biocatalyst called the $\mathrm{Mn}_{4} \mathrm{O}_{5} \mathrm{Ca}$ cluster. The precise structure of this $\mathrm{Mn}_{4} \mathrm{O}_{5} \mathrm{Ca}$ cluster has been determined quite recently $[9,10]$ and has attracted a lot of attention from the artificial photosynthesis community. This is because the structure forms a blueprint for an artificial biocatalyst that can abstract electrons and protons $\left(\mathrm{H}^{+}\right.$ion $)$from water, which is one of the most table compounds on Earth. A further important point of the oxygen evolving type photosynthesis is that it allows the utilization of light from a broad spectral range, from ultraviolet to infrared, to produce high oxidation and reduction potentials at the same time through the connection of the two independent RCs, PSI and PSII (this interrelationship is called the Z-scheme).

A big challenge of artificial photosynthesis is to produce a fuel from sunlight by 
mimicking the reaction of photosynthesis. To this end, as illustrated in Figure 2, the above-mentioned photosynthesis reactions can be divided into four interdependent elements and then the production of solar fuels may become possible. The four elements are (1) a light-harvesting antenna element that can capture the solar energy and transfer the captured energy efficiently to the RC, (2) a RC element that can produce a sufficiently high electromotive force for oxidation and reduction reactions, (3) an oxidation catalyst element that can split water to produce electrons and protons, (4) a reduction catalyst element that can utilize these electrons and protons to produce a solar fuels (by, for example, reducing $\mathrm{CO}_{2}$ ). In the following sections, the present status of the advanced studies about these four elements is discussed.

\subsection{Why a Light-Harvesting Antenna is Important?}

The gross amount of light from the sun that reaches the Earth is vast. However, this is not a large value numerically when converted into photon flux density in a unit time per unit area $[11,12]$. This becomes clear when the exact calculation is performed. The quantity of the solar energy that hits the surface of the Earth is prescribed with a solar constant and is approximately $1.37 \mathrm{~kW} / \mathrm{m}^{2}$. When converting this amount of energy into the number of photons, it becomes as large as or greater than $6 \times 10^{21} / \mathrm{m}^{2} \mathrm{~s}$. The number of the photons within the $400-800 \mathrm{~nm}$ spectral region (visible light domain) is $1.6 \times$ $10^{21} / \mathrm{m}^{2} \mathrm{~s}$. This is equivalent to only a $1 / 400$ of the Avogadro's number for one second per 1 square meter. If all of these photons can be utilized with $100 \%$ efficiency, then only $11 \mathrm{mg}$ of water can be split because four photons are necessary to split one molecule of water. In other words, the gross amount of solar energy is vast, but radiation density has a certain limit. Therefore, accumulation of photons (antenna function) becomes important.

Plant photosynthesis has been developing unique light harvesting antenna systems to efficiently collect light energy from the sun to overcome this problem. Various phototrophs such as higher plants, algae, and photosynthetic bacteria inhabit the Earth. As illustrated in Figure 3, if the absorption spectra of all of the antenna systems from these phototrophs are overlaid they reveal an almost complete coverage of the solar radiation spectrum. In other words, phototrophs on the earth have evolved so that they can 'share' the solar radiation by developing their own unique antenna systems with 
characteristic absorption bands. Learning this wisdom and replicating this wonder of Nature may hold the key to the development of a highly efficient light-harvesting system that is one of the bottlenecks towards the full utilization of solar energy, not just in artificial photosynthesis but also for other forms of solar energy conversion.

These phototrophs have various colors due primarily to the presence of the class of pigments called carotenoids that absorb within the visible region. Therefore, it is not too much of an overstatement to say that the correct understanding of the functions of carotenoids relates directly to the complete understanding of photosynthetic light-harvesting. In the primary process of photosynthesis, carotenoids transfer their captured solar energy to enhance that of $\mathrm{Chl}$ alone. This excitation energy initiates a photosynthetic multi-step reaction with more than 40 steps. Surprisingly each process proceeds with the efficiency of nearly $100 \%$. However, the efficiency of energy transfer from carotenoid to Chl varies from $30 \%$ to nearly $100 \%$ depending on the structure of the molecule in question. Therefore, understanding the functions of carotenoids holds a key towards the effective utilization of solar energy.

\section{Architecture of Natural Photosynthetic Systems}

\subsection{Overview of Natural Photosynthetic Systems}

As alluded to previously, photosynthesis is not only found in plants and algae but also in certain classes of bacteria. Though these organisms can be strikingly different, their basic pattern of light reactions is quite similar. They all contain light-harvesting systems that are coupled to RCs. The majority of the light-harvesting pigments (mainly different types of Chls, carotenoids, and phycobilins) are organized into light-harvesting complexes. A small proportion of the Chls and carotenoids are present in the RCs where the absorbed light energy is initially converted into chemical energy. Whereas the structures of the RCs are all rather similar, those of the light-harvesting complexes are bewilderingly variable. Once the RCs have used the absorbed solar energy to drive the initial electron transfer reactions, subsequent electron transfer takes place within the photosynthetic membranes that finally result in the production of ATP and reduced $\mathrm{NAD}(\mathrm{P})$. These two compounds are then consumed in metabolic reactions where 
carbohydrate is produced [13-15]. The 'light' reactions typically take place in and on the photosynthetic membranes, while the 'dark' (actually non-light dependent) reactions take place in the aqueous phase, either in the stroma of the chloroplast or the cytoplasm of the photosynthetic cells.

Figure 4 shows the schematic illustration of the structure and pigment composition of the major types of photosynthetic antenna pigment-protein complexes. It should be pointed out that although most antenna complexes involve proteins as well as the pigments, this is not the case for chlorosomes, which are highly organized non-protein pigment only complexes.

As shown in Figure 4 anoxygenic photosynthetic organisms only contain one type of RC. Green sulfur bacteria typically contain a so-called type I RCs that have iron-sulfur complexes as their terminal electron acceptors. The purple bacteria contain type II RCs that have quinones as their terminal electron acceptors. Oxygenic photosynthetic organisms have both types of RC, in this case usually called PSI and PSII. The light-harvesting complexes are arranged around the RCs to act as an antenna by collecting and funneling the harvested excitons down an energy gradient towards the RC. Often these light-harvesting complexes can be thought of consisting of two basic functional types, the core and the peripheral light-harvesting complexes. Usually all RCs have core light-harvesting complexes associated with them that form a minimum light-harvesting unit. Then surrounding these units the peripheral light-harvesting complexes are arranged. The type and the amount of these peripheral light-harvesting complexes are controlled by a variety of environmental factors such as light intensity and light quality. Typically, the size of the peripheral light-harvesting systems becomes larger as the light intensity becomes lower.

X-ray crystallography has been a powerful tool with which to determine the structure of the photosynthetic pigment protein complexes. The first structure of Chl containing protein was that of the water-soluble Fenna-Matthews-Olson (FMO) protein [16] from the green sulfur bacterium Chlorobium limicola. However, since most of the important photosynthetic pigment-protein complexes containing Chls and carotenoids are membrane proteins, there was a long delay between the description of the structure of FMO and the determination of the high-resolution structure of the membrane bound purple bacterial RC. The first example of the X-ray crystallography of the RC from Blastochloris (Blc.) viridis was reported in 1985 [17]. This pioneering work carried out 
by three German researchers, Michel, Deisenhofer and Hüber and was recognized by the award of the Nobel Prize for Chemistry in 1988. This achievement broke the logjam so that we now have high resolution structures of PSI [18] and PSII [9, 10] from cyanobacteria, LHCII from higher plants [19] and LH2 [20, 21] and RC-LH1 core complexes [22-24] from purple bacteria. These structures are all illustrated in Figure 5. Also, remarkably, in recent years high-resolution transmission electron microscopy (TEM) and atomic force microscopy (AFM) have been used to visualize the organization of these complexes within fully native photosynthetic membranes [25-27]. As a result of these we now have both a detailed structural picture of the individual complexes and well-defined maps of how these complexes interact with each other within the photosynthetic membranes.

Carotenoids are found in all of the pigment-protein complexes shown in Figure 5, both in the RCs and the light-harvesting complexes [3, 4]. Table 1 describes the carotenoid composition of some of the different RCs and light-harvesting complexes. In oxygenic photosynthetic organisms the RCs and core complexes mainly contain $\beta$-carotene, whereas the carotenoid composition of the peripheral light-harvesting complexes is more varied. For example, the higher plant LHCII complexes mainly contain a mixture of lutein, neoxanthin, zeaxanthin, and violaxanthin. The peripheral antenna complexes found in heterokontophyta, including diatoms and brown algae, contain carotenoids such as fucoxanthin that function as a much more efficient accessory light-harvesting pigment than those of other carotenoids that have similar absorption region with fucoxanthin in the proteins, the details of which are described in the following section. This is somewhat to be expected as this group of photosynthetic organisms essentially obtains all of their light energy from that absorbed by carotenoids. A similar situation is found in dinoflagellates where their major light-harvesting complex, the peridinin-Chl $a$ complex, also uses another unique carotenoid that is highly efficient at transferring energy to the Chls. In this case the peridinin-Chl $a$ complex is water-soluble and its structure has previously been determined by X-ray crystallography [28]. The peridinin-Chl $a$ complex is rather unusual as it contains more carotenoids than Chls. The red algae and cryptophytes also contain water-soluble antenna complexes. These are called phycobiliproteins and contain no chlorophylls or carotenoids. Their light absorbing pigments are a group of open chain tetrapyrroles called phycobilins. Interestingly these are the only examples of light-harvesting 
pigments that are covalently attached to the pigment binding proteins. The phycobiliproteins are typically organized into large multi-subunit structures called the phycobilisomes. The final type of light-harvesting system is present in the green sulfur bacteria. These bacteria contain chlorosomes that are like "bags" surrounded by a lipid monolayer and containing protein free self-aggregate of bacteriochlorophyll (Bchl) $c, d$, and $e$. These Bchls form strongly exciton-coupled aggregates that absorb solar energy and transfer it via a base plate structure onto the membrane bound RCs. In the green sulfur bacteria it is the previously mentioned FMO protein that mediates the energy transfer from the base plate to the RCs.

\subsection{Marine Algae}

On Earth the sea covers approximately $70 \%$ of the total surface area and it is to be expected, therefore, that many organisms live photosynthetically in this vast oceanic area. In fact oceanic photosynthetic organisms, primarily marine algae, contribute almost one quarter of global primary photosynthetic production [2]. The marine algal photosynthetic systems are similar to those of cyanobacteria and higher plants, in that they perform oxygenic photosynthetic light reactions; a schematic illustration is shown in Figure 6. These series of reactions are common for cyanobacteria and higher plants, however, the characteristic point in marine algae is that they have developed unique antenna systems for the purpose of adjusting to the fluctuating light conditions within their marine habitat. The antenna in these organisms often contains a $\mathrm{Chl} c$ in addition to the general $\mathrm{Chl} a$ [29]. The amino acid sequences of the $\mathrm{Chl} a / c$ type antennae are similar to that of the well-known peripheral Chl $a / b$ type antenna in higher plants, LHCII, although a high-resolution X-ray structure has not yet been obtained [29]. One of the major Chl a/c type antennae is "fucoxanthin-Chl a/c protein" (FCP) found mainly in brown algae and diatoms. In the FCP, both fucoxanthin and $\mathrm{Chl} c$ collect the light energy and transfer it to $\mathrm{Chl} a$, then this is followed by the energy transfer to the RCs (both photosystems I and II) where charge separation takes place. In order to elucidate the detailed mechanism of the energy transduction, excited-state properties of all the photosynthetic pigments involved in the primary process should be investigated, however, the study on the excited-state properties of Chl $c$ left behind [29].

A study on FCP from microalgae has only been performed relatively recently [30], 
due primarily because of the difficulty of reliable and consistent sample preparations. However, there is some progress to overcome this drawback using a species of Nemacystus decipiens. Cladosiphon okamuranus, "Okinawa Mozuku" in Japanese, is an original strain of a brown alga found in Okinawa, Japan. Super large-scale culture of this strain has been established in the "discoid germiling" form. The discoid germiling is a beneficial source of photosynthetic apparatus because it is a micron-size cell body without any of the normally associated resilient lipids and polysaccharides. Therefore, studies using this form of the strain provide a more promising pathway to elucidate the structure and function of the FCP and purification methodology of the "Mozuku FCP" has already been established [31]. Subunit analyses and amino acid sequencing using TOF-MS/MS spectrometry have been performed to determine the proteomics of the "Mozuku FCP". Nondestructive electrophoresis (Native-PAGE), SDS-PAGE and two-dimensional electrophoresis in addition to the results of quantitative gel filtration HPLC all suggest that "Mozuku FCP" is hetero-trimer, composed of two subunits of $17.5 \mathrm{kD}$ and $18.2 \mathrm{kD}$, respectively [31].

It is believed that the carotenoid fucoxanthin as well as $\mathrm{Chl} c$, peculiar to oceanic phototrophs, are bound to "Mozuku FCP" in addition to Chl $a$ (see Figure 6). Specifically, it has been thought that only $\mathrm{Chl} c_{2}$ was bound as $\mathrm{Chl} c$. This is because $\mathrm{Chl} c$ is an organic acid, and it has been particularly difficult to separate $\mathrm{Chl} c c_{1}$ and $\mathrm{Chl}$ c2. We have succeeded in performing a total analysis of "Mozuku FCP", including Chl $c_{1} / \mathrm{c}_{2}, \mathrm{Chl} a$ and carotenoid, for the first time using Inertsil ODS-P column [32]. The stoichiometry of these pigments was determined using ${ }^{1} \mathrm{H}-\mathrm{NMR}$ spectroscopy. Previous spectroscopic studies on FCP have mainly been performed for that isolated from diatoms [33-37]. In this system an energy deactivation process called the diadinoxanthin cycle exists and inhibits efficient energy transfer from fucoxanthin to Chls [30]. However, the "Mozuku FCP" is an ideal system for artificial photosynthesis, as it does not have this unwanted energy dissipation process and achieves nearly $100 \%$ fucoxanthin to Chls energy transfer efficiency [38].

\section{Purple photosynthetic bacteria as a model system with which to understand the overall energy and electron transfer reactions that occur in the early stages of photosynthesis}


In this section the basic concepts of energy and electron flow in the primary processes of photosynthesis are outlined from purple bacteria [15]. The light reactions in purple photosynthetic bacteria take place in and on an extensive system of intra-cytoplasmic membranes (ICM). In bacterial genera like Rhodobacter (Rba.) sphaeroides and Rhodospirillum (Rsp.) rubrum the ICM are vesicular membranes (individual vesicles are called chromatophores), while other genera such as Rhodoblastus (Rbl.) acidophilus and Blc. viridis the ICM form flat, lamellae membranes. Figure 7 shows an absorption spectrum of chromatophores of Rba. sphaeroides strain 2.4.1 recorded at room temperature. Structured absorption bands are spread over a wide spectral range including the ultraviolet, visible and near infrared regions. They are all due to two types of photosynthetic pigments, either carotenoid or Bchl $a$. Figure 8 shows the chemical structures of the main pigments, carotenoid and Bchl $a$, that are bound to the Rba. sphaeroides strain 2.4.1 photosynthetic complexes. In Figure 7 the carotenoid absorption band, annotated with Car, is due to spheroidene and those annotated with $\mathrm{B}, \mathrm{Q}_{\mathrm{x}}$, and $\mathrm{Q}_{\mathrm{y}}$ are due to Bchl $a$. Carotenoids are hydrocarbons with a linearly conjugated polyene chain (see Figure 8(a)). The positions of their absorption bands depend on the length of conjugation. Since in purple bacteria the major Bchl $a \mathrm{Q}_{\mathrm{y}}$ absorption bands lie outside the color range seen by our eyes, the differences in the conjugation length of the carotenoids are the reason for the difference of colors of purple photosynthetic bacteria (see Figure 12, vide infra). Figure 8(b) shows the chemical structure of Bchl $a$. All of the optical and photophysical properties of the molecule reside in the delocalized, conjugated bonds that form the bacteriochlorin ring. The phytol chain is chemically and photochemically inert. The exact position of the $\mathrm{Q}_{\mathrm{y}}$ absorption band of Bchl $a$ is reflective of and sensitive to the non-covalent binding of the pigment within the light-harvesting apoproteins. Three distinct $\mathrm{Q}_{\mathrm{y}}$ absorption bands of Bchl $a$ are observed at 800,850 and $880 \mathrm{~nm}$. These pigment bands can be attributed to Bchl a molecules are non-covalently bound in the same way to their respective light-harvesting apoproteins. In general, all purple photosynthetic bacteria have two major pigment-protein complexes; the light-harvesting 1 (LH1) antenna complex and the RC. The LH1 surrounds the RC complex to form the core antenna. Most species of purple photosynthetic bacteria contain a second, more peripheral (to the $\mathrm{RC}$ ) antenna complex called light-harvesting complex 2 (LH2). The antenna pigment-protein 
complexes capture light energy and transfer it to the $\mathrm{RC}$, in the form of excitation energy. The energy flows in an energy gradient 'downhill' from LH2 $\rightarrow$ LH1 $\rightarrow$ RC (see Figure 10, vide infra). In the RC charge separation takes place using thus transferred excitation energy, and electron transfer reactions follow as a consequence.

Figure 9 shows the pigment arrangement determined by X-ray crystallography of the RC from Rba. sphaeroides [39]. Here $\mathrm{P}$ is a 'special pair' Bchl $a$ dimer, B is a Bchl $a$ monomer, $\mathrm{H}$ is bacteriopheophytin (Bphe), and $\mathrm{Q}$ is ubiquinone. These pigments are arranged with pseudo two-fold symmetry in the RC. The excitation energy is transferred from LH1 complexes to the Bchl $a$ dimer $(\mathrm{P})$. Then the electron transfer reactions follow. Although the pigments are arranged into two arms that show pseudo two-fold symmetry, electron transfer only takes place down the pathway that is denoted with subscript "A" (A-branch). An electron leaves $\mathrm{P}$ and is transferred via $\mathrm{B}$ and $\mathrm{H}$ arriving at $\mathrm{Q}_{\mathrm{A}}$ within 200 ps after charge separation. One carotenoid molecule is also bound to the RC and has 15-cis conformation. This carotenoid breaks the two-fold symmetry and lies beside the inactive B-branch. This carotenoid molecule has the crucial function of protecting the $\mathrm{RC}$ from destructive photo-damage when excess amounts light energy are present (photoprotective function) [40]. The details of this photoprotective reaction will be described below.

The electron on $\mathrm{Q}_{\mathrm{A}}$ is eventually transferred to $\mathrm{Q}_{\text {в. }} \mathrm{Q}_{\text {в }}$ is capable of accepting two electrons. Then $\mathrm{QB}^{2-}$ binds with two protons $\left(\mathrm{H}^{+}\right)$and generates $\mathrm{Q}_{\mathrm{B}} \mathrm{H}_{2}$. This ubiquinol form is no longer bound by the RC and diffuses out of the RC pocket. As illustrated in Figure 10, $\mathrm{Q}_{\mathrm{B}} \mathrm{H}_{2}$ released from the $\mathrm{RC}$ is transferred into quinone pool that exists within the photosynthetic membranes. The fully reduced quinone diffuses in the plane of the membrane to the cytochrome $b c_{1}$ complex where it is oxidised back to Q $\mathrm{B}$. From cytochrome $b c_{1}$ complex the released electrons are subsequently used to reduce cytochrome $c_{2}$. This cytochrome $c_{2}$ carries the electron back to the RC and re-reduces $\mathrm{P}^{+}$ to P. During this process of cyclic electron transport, protons are pumped across the membrane generating a proton motive force $(\Delta \mathrm{pH})$. This proton gradient is then utilized by ATP synthase to generate ATP by the classical chemiosmotic mechanism. X-ray crystallographic structures have now been determined for cytochrome $b c_{1}$ [41], cytochrome $c_{2}$ [42], and ATPase [43].

The first high-resolution X-ray crystallographic structure of a membrane bound antenna pigment-protein complex was published 10 years after that of the RC [20]. 
Figure 11 (right-hand side) shows the structure of the LH2 complex from the purple photosynthetic bacterium Rbl. acidophilus strain 10050. The LH2 complex is an oligomer of a monomer unit that is composed of a pair of $\alpha$-helical apoproteins ( $\alpha$-polypeptide (inner) and $\beta$-polypeptide (outer)) in which one Bchl $a$ monomer (B800 $\mathrm{Bchl} a$ ), one Bchl $a$ dimer (B850 Bchl $a$ 's), and one carotenoid (rhodopin glucoside) are non-covalently bound. Nine of these monomer units aggregate to form a beautiful ring structure with 9-fold symmetry. As shown in Figure 7 and 12, there are two populations of Bchl $a$ that are present in a single LH2 complex and have $\mathrm{Q}_{\mathrm{y}}$ absorption maxima at $800 \mathrm{~nm}$ and $850 \mathrm{~nm}$. Within this hetero dimeric sub-unit the monomeric Bchl $a$ gives rise to the $800 \mathrm{~nm}$ absorption band, and the dimeric Bchls $a$ give rise to the $850 \mathrm{~nm}$ absorption band. Dimerization of Bchl $a$ molecules stabilizes the excited state energy by excitonic interaction. This excitonic interaction of the $850 \mathrm{~nm}$ Bchls extends throughout all nine heterodimer units to form an excitonically coupled ring within the complex. This ring of excitonically coupled Bchl accounts for the $\mathrm{Q}_{\mathrm{y}}$ absorption maximum exhibiting a $50 \mathrm{~nm}$ red-shift compared to that of monomer. The carotenoid that is bound to LH2 is in van der Waals interaction with both the B800 and B850 Bchls. The carotenoid absorbs the blue-green region of sunlight where Bchl $a$ has virtually no absorption, and transfers the captured energy to both B800 and B850 Bchls (light-harvesting function of carotenoids). The detailed mechanisms involved in these energy transfer reactions will be described later in this review. The carotenoid molecule intercalates between neighboring monomer units of LH2 leading to stabilization of the nonamer structure [40]. In many cases if the carotenoid is not present then the LH2 complexes will fail to assemble.

Following the determination of the structure of LH2 a low-resolution projection map of LH1 (a reconstituted sample from Rsp. rubrum) showed that it was a larger ring structure (a 16-mer) [44]. The first low resolution X-ray crystal structure of an LH1-RC core complex from a purple bacterium was described in 2003 [22]. This complex was obtained from Rps. palustris and structure was determined at a resolution of $4.8 \AA$. This structure is illustrated in Figure 11 (left hand side). The LH1 complex is composed of 15 pairs of transmembrane $\alpha$-helical $\alpha$ - and $\beta$-polypeptides and one transmembrane $\alpha$-helical polypeptide (denoted W in Figure 11). The LH1 ellipse surrounds the RC. The W polypeptide is thought to be a PufX homologue and to prevent the closure of the LH1 ellipse, thereby providing a gap through which the $\mathrm{Q}_{\mathrm{B}} \mathrm{H}_{2}$ released from the $\mathrm{RC}$ can 
diffuse through the LH1 complex and enter the bulk membrane phase to connect with the other components of the cyclic electron transport pathway. More recently a $3.0 \AA$, high-resolution X-ray crystallographic structure of the LH1-RC complexes from Chromatium tepidum has been reported, in which the LH1 ring is continuous and a $\mathrm{W}$-like protein is absent [24]. In this case the $\mathrm{Q}_{\mathrm{B}} / \mathrm{Q}_{\mathrm{B}} \mathrm{H}_{2}$ exchange takes place through the LH1 helical palisade and is facilitated by the vibrational motion of the complex. In addition, a low resolution model structure of the dimeric PufX-LH1-RC complex from Rba. sphaeroides is now also available [23].

The development of ultrafast laser spectroscopies has allowed real time observation of the excitation energy transfer (EET) in light-harvesting complexes to become possible [45]. In Figure 11 typical rate constants of the EET in a purple bacterial photosynthetic unit have been summarized. The rates of EET are very fast. This is essential since a typical lifetime for a Bchl $a$ first excited singlet state is about one nanosecond $\left(10^{-9} \mathrm{~s}\right)$. If any EET process is going to be efficient, then its rate must be much faster than this timescale. The rate of EET from B800 to B850 in LH2 is 0.9 ps at room temperature. It then takes 3-5 ps for the energy to be transferred from B850 in LH2 to B875 in LH1. The slowest step in the EET pathway is from B875 in LH1 to the special in the RC. This step takes about 35 ps. The slowness is due to the longer distance involved in this final EET step. It should be pointed out though that the total transfer time from B800 in LH2 to the special in the RC only takes about 40 ps and this is sufficiently faster than one nanosecond and so ensures high efficiency.

\section{An outline of ultrafast relaxation processes of carotenoids following photoexcitation}

Carotenoids have strong absorption of visible light in the blue and green region of the spectrum (Figure 12). Most of the carotenoids found in photosynthetic organisms have characteristic yellow, orange, and red colors. The lowest excited singlet-state in most pigment molecules represents the lowest energy, optically allowed one-photon transition from the ground state. The energy of this state controls the color of that pigment molecule. However, carotenoids have a non-standard pattern of excited states. The lowest energy optically allowed excited singlet-state is not the lowest energy 
singlet-state. The lowest singlet excited energy state is formally a one photon forbidden state. This unusual photophysical pattern is a result of symmetry rules. The ground electronic state of the carotenoid has ${ }^{1} \mathrm{Ag}^{-}$symmetry based on the assumption that it has a $\mathrm{C}_{2 h}$ point symmetry in its polyene backbone. The lowest singlet excited state, $\mathrm{S}_{1}$ has $2^{1} \mathrm{Ag}^{-}$symmetry and a one photon induced transition is, therefore, optically forbidden. The lowest optically allowed state is the $1^{1} \mathrm{~B}_{\mathrm{u}}{ }^{+}\left(\mathrm{S}_{2}\right)$ state. When the $1^{1} \mathrm{~B}_{\mathrm{u}}^{+}$state is induced by a short excitation pulse it decays into the $2^{1} \mathrm{Ag}^{-}$state within $100-300 \mathrm{fs}$. The $S_{1}$ state typically decays back to the ground state in a few picoseconds [46]. The exact rate constant of these processes depends upon factors such as the number of conjugated double bonds $(n)$. This, however, is probably an over simplification as theoretical studies based primarily on symmetrical polyenes have predicted other possible excited singlet states such as $3^{1} \mathrm{Ag}^{-}$and $1^{1} \mathrm{~B}_{\mathrm{u}}{ }^{-}[47,48]$. The possibility of these multiple excited states, and indeed others, has made understanding carotenoid photophysics extremely complicated [49].

In the following discussion of the light-harvesting role of carotenoids in pigmentprotein complexes from purple bacteria a simplified view will be initially presented and then introduce some of the complications to try and interpret where the field is currently. Probably the simplest way to begin this discussion is to describe the experiments of Macpherson et al. [50]. These authors compared the excited state kinetics of a carotenoid in organic solvent with the same carotenoid when it was bound within a LH2 complex. The idea was that any carotenoid singlet excited state that was capable of transferring energy to Bchl $a$ in LH2 would be expected to have a shorter excited state lifetime in the antenna complex than in the organic solvent, since the energy transfer pathway would open another decay channel, thereby, accelerating the overall rate of decay of that excited singlet state. Moreover, it would be expected that the decay of the donor carotenoid excited singlet state should match the rate of the energy arriving at the Bchl $a$ molecule. In the case of the carotenoid rhodopin glucoside the decay of the $\mathrm{S}_{2}$ state in the LH2 complex from Rbl. acidophilus was faster than that of the carotenoid in organic solvent (56 fs in LH2 and 133 fs in benzyl alcohol). The rise time of the arrival of the energy at both B800 and B850 matched decay time of rhodopin glucoside's $\mathrm{S}_{2}$ state. The decay rate of the $S_{1}$ state of rhodopin glucoside was the same in the LH2 complex as in organic solvent. In this case the $\mathrm{S}_{1}$ state is not active in energy transfer to Bchl $a$. However, the LH2 complex from Rba. sphaeroides contains carotenoids with 
fewer conjugated double bonds and in this case the $S_{1}$ state is able to transfer energy to the Bchl $a$ molecules and its decay is accelerated in LH2 compared with organic solvent [51].

These experiments were performed when the excitation pulses were relatively long, however, recently it has become possible to use much shorter femtosecond pulses. When this was done by Cerullo et al. the data showed the presence of an intermediate state between $S_{2}$ and $S_{1}$ [52]. This state was formed as $S_{2}$ decayed and gave rise to $S_{1}$ as it decayed. However with these extremely fast reactions it was not possible to be sure that this intermediate state was another pure excited singlet state such as $1^{1} \mathrm{~B}_{\mathrm{u}}{ }^{-}$. Since this time there have been many studies that have been suggested to require such an intermediate state to fully explain the experimental data. Therefore, the appearance of a nonlinear optical effect was suspected [53], yet even this is not the complete picture. There have been further studies that have not seen or required the presence of such extra intermediate state to fit the data [54]. This has led to a lot of confusion. Most recently broadband two-dimensional (2D) electronic spectroscopy measurements on light-harvesting proteins from purple bacteria and isolated carotenoids were performed in order to characterize in more detail the excited-state manifold of carotenoids that channel energy to Bchl molecules. The data revealed a well-resolved signal (cross peak) consistent with a previously postulated carotenoid dark state, the presence of which was confirmed by global kinetic analysis. The results suggest that the carotenoid dark state has a role in mediating energy flow from carotenoid to Bchl [55].

Another type of intermediate excited state, termed as $\mathrm{S}^{*}$ has been found with carotenoids both free in solution and bound to light-harvesting complexes, revealing a further level of complication [56-60]. At the higher-energy side of the $\mathrm{S}_{1} \rightarrow \mathrm{S}_{\mathrm{n}}$ transition, a new transient absorption band was detected by means of pump-probe time-resolved absorption spectroscopy and subsequent spectral analysis using SVD (singular value decomposition) and global fitting. This newly identified absorption band was assigned to the $\mathrm{S}^{*}$ state. The lifetime of this particular state was determined to be between 5 and 12 ps depending on both the species of carotenoid and whether it was present in the light-harvesting complex or in organic solvent. The $\mathrm{S}^{*}$ state decayed into the triplet state when the carotenoid was bound to the LH2 complex. However, when the carotenoid was free in organic solvent the $S^{*}$ state decayed to the ground state without generating the triplet state. Applying a pump-dump and transient absorption technique 
for $\beta$-carotene, lycopene, and zeaxanthin, Wohlleben et al. re-examined the origin of the $\mathrm{S}^{*}$ state with the carotenoid free in solution $\left(\mathrm{S}^{*}\right.$ sol) [59]. They suggested that the $\mathrm{S}^{*}$ sol state is a vibrational excited ground-state $\left(\mathrm{S}_{\text {sol }}=\right.$ hot $\left.\mathrm{S}_{0}\right)$, which is populated by a combination of impulsive Raman scattering of the pump pulse and $\mathrm{S}_{1} \rightarrow \mathrm{S}_{0}$ internal conversion. They also found the $\mathrm{S}^{*}$ state of the protein-bound carotenoid and re-designated it as $\mathrm{S}^{*}$ T. These ideas have recently been supported by the present author's groups for spirilloxanthin both free in solution and bound to light-harvesting complexes [60].

Involvement of vibrationally excited states in the relaxation process of carotenoids after photoexcitation was initially detected by time-resolved absorption spectroscopy [61-63], and has also been studied by time-resolved stimulated Raman spectroscopy [64-68]. This important issue is further discussed in the following sections.

For carotenoids that contain carbonyl groups there is the possibility of forming intramolecular charge-transfer states (SICT). These states have been well documented in the case of carotenoids such as peridinin and fucoxanthin (see Figure 13 for chemical structures of these molecules) $[38,64,65,69-75]$. The importance of this charge transfer state seems to be that it allows carotenoid to chlorophyll energy transfer to be highly efficient. Readers who are interested in more details about this state should consult the excellent review by Polívka and Sundström [76]. In our recent study, it was demonstrated that a large part of excitation energy captured by fucoxanthin bound to FCP ("Mozuku FCP") is transferred to Chl a via the coupled $\mathrm{S}_{1} / \mathrm{ICT}$ state, resulting from a strong electronic dipole interaction between fucoxanthin and Chl $a$ [38]. This strong dipole interaction is attributed to an ICT character in the excited state of fucoxanthin, enabling it to enhance the transition dipole moment of the $\mathrm{S}_{1} / \mathrm{ICT}$ state. On the other hand, the nature and origin of the $\mathrm{S}_{1} / \mathrm{ICT}$ state of carbonyl carotenoids has not yet been fully understood. Therefore, we have investigated the ultrafast excited state dynamics of fucoxanthin and its homologues, the chemical structures of which are illustrated in Figure 14.

The spectroscopic properties of fucoxanthin in polar (methanol) and nonpolar (cyclohexane) solvents were studied [72]. Transient absorption associated with the optically forbidden $\mathrm{S}_{1}\left(2^{1} \mathrm{Ag}^{-}\right)$and/or the ICT states were observed following one-photon excitation to the optically allowed $\mathrm{S}_{2}\left(1^{1} \mathrm{Bu}^{+}\right)$state in methanol. The transient absorption measurements carried out in methanol showed that the ratio of the ICT-to-S ${ }_{1}$ state 
formation increased with decreasing excitation energy. The ICT character was clearly visible in the steady-state absorption in methanol based on a Franck-Condon analysis. The results suggest that two spectroscopic forms of fucoxanthin, blue and red, exist in the polar environment. The spectroscopic properties of fucoxanthin in methanol were further studied by femtosecond pump-probe measurements in the near-infrared region, where transient absorption associated with the optically allowed $\mathrm{S}_{2}\left(1^{1} \mathrm{~B}_{\mathrm{u}}{ }^{+}\right)$state and stimulated emission from the strongly coupled $\mathrm{S}_{1} / \mathrm{ICT}$ state were observed following one-photon excitation to the $S_{2}$ state [74]. The results showed that the amplitude of the stimulated emission of the $\mathrm{S}_{1} / \mathrm{ICT}$ state increased with decreasing excitation energy, demonstrating that the red-form of fucoxanthin exhibits a stronger ICT character. A magnitude of ICT character of carbonyl carotenoids has often been evaluated by (1) solvent polarity-dependent $\mathrm{S}_{1} / \mathrm{ICT}$ lifetimes, (2) amplitudes of the ICT transient absorption and stimulated emission bands, and (3) a dipole moment of the ICT state. Femtosecond pump-probe spectroscopic measurements were performed on fucoxanthin homologs with varying numbers of conjugated double bonds $(n=4 \sim 8)[75,77]$. The ICT properties of fucoxanthin homologs were characterized by the $\mathrm{S}_{1} / \mathrm{ICT}$ lifetimes and the transient absorption and stimulated emission bands due to the $\mathrm{S}_{1} / \mathrm{ICT}$ state.

Figure 13 shows a schematic illustration of the relative energies of the carotenoid excited singlet states discussed above together with the proposed relaxation pathways from the $\mathrm{S}_{2}$ state as well as the energy-transfer pathways between carotenoid and Bchl. Since the relaxation from the $\mathrm{S}_{2}$ state is very fast, ultrafast vibrational spectroscopies are going to be important to clarify the structure-function relationship of the above singlet excited-states [78]. Therefore, this important issue will be extensively reviewed in the following sections.

\section{Time-Resolved Raman and Two-Photon Excitation Spectroscopies of Carotenoids}

Figure 15 shows the steady-state absorption spectrum of all-trans- $\beta$-carotene (see Figure 13 for its chemical structure). The absorption peak that corresponds to optically allowed $1^{1} \mathrm{Bu}^{+}\left(\mathrm{S}_{2}\right)$ singlet excited-state appears at $2.55 \mathrm{eV}(486 \mathrm{~nm})$. The peaks appear at $2.73 \mathrm{eV}(455 \mathrm{~nm})$ and $2.90 \mathrm{eV}(428 \mathrm{~nm})$ are side bands due to molecular vibrations. 
In the case of carotenoids these side bands are mainly due to $v_{1}\left(\sim 1500 \mathrm{~cm}^{-1}, \mathrm{C}=\mathrm{C}\right.$ stretching) and $v_{2}\left(\sim 1200 \mathrm{~cm}^{-1}, \mathrm{C}-\mathrm{C}\right.$ stretching $)$ modes. The lowest excited singlet $\left(\mathrm{S}_{1}\right)$ state of carotenoids is lying in energy below the $\mathrm{S}_{2}$ state. This $\mathrm{S}_{1}$ state is designated as $2^{1} \mathrm{Ag}^{-}$, which has the same symmetric character with the ground $\left(\mathrm{S}_{0}\right)$ state, and hence the transition from the $\mathrm{S}_{0}$ state is one-photon forbidden. Therefore, the $\mathrm{S}_{0} \rightarrow \mathrm{S}_{1}$ absorption cannot be detected in a conventional steady-state absorption spectrum. However, carotenoids somewhat break their symmetry in solution and very weak fluorescence can be observed [79]. The absorption spectrum of the $2^{1} \mathrm{Ag}^{-}\left(\mathrm{S}_{1}\right)$ state shown in Figure 15 was obtained by the spectral simulation using the parameters determined by fluorescence spectroscopy.

\subsection{Time-resolved Raman gain and loss spectroscopies}

Raman spectroscopy is a spectroscopic technique to investigate the molecular vibrations using the energy difference between the excitation laser light and the scattered radiation. Time-resolved Raman spectroscopy can be utilized to investigate the time evolution of molecular vibrations, structures, and the structural change of molecules in the excited electronic states. Time-resolved Raman spectroscopy on polyene molecules clarified that the $v_{1}(\mathrm{C}=\mathrm{C}$ stretching $)$ mode in the $2^{1} \mathrm{Ag}^{-}\left(\mathrm{S}_{1}\right)$ state shows unusually high frequency compared to that in the ground state [80]. In general, molecular vibrations in the excited electronic states are expected to have lower frequencies than their counterparts in the ground electronic state because of the expansion of wave functions in the excited electronic states. However, polyene molecules do not obey this simple rule. This anomaly can be explained by strong vibronic coupling between the ground $1{ }^{1} \mathrm{Ag}^{-}\left(\mathrm{S}_{0}\right)$ and the lowest excited singlet $2^{1} \mathrm{Ag}^{-}$ $\left(\mathrm{S}_{1}\right)$ states [80]. Similarly, in the case of carotenoids picosecond time-resolved Raman spectroscopy has clarified that the $v_{1}$ mode in the $2^{1} \mathrm{Ag}^{-}\left(\mathrm{S}_{1}\right)$ state appears at extraordinary high frequency $[81,82]$. According to the development of ultrafast laser systems, it becomes easier to observe the ultrafast phenomena with temporal resolution in femtosecond time regime. As a consequence, it had been expected to reveal the detailed mechanism that produces the anomalous vibrational behavior of the $2^{1} \mathrm{Ag}^{-}$state of carotenoids. However, using conventional methods, it is difficult to obtain satisfactory frequency resolution for Raman spectroscopy due to the uncertainty 
principle of quantum mechanics. Femtosecond time-resolved Raman spectroscopy can become feasible using the process of stimulated Raman scattering [83-86].

Three laser pulses are used in the femtosecond stimulated Raman spectroscopy and Figure 16 shows an outline schematic. The first laser pulse (frequency, $\omega_{e x}$ ) excites the molecule to it's excited electronic state (e). The target excited-state for Raman measurement is then populated following the relaxation process. In Figure 16 it is assumed that the vibrational ground $(l=0)$ and excited $(l=1)$ states in the electronic excited state, respectively, have the population $N_{0}$ and $N_{1}$. If Raman excitation laser pulse (frequency, $\omega_{R}$ ) and probe light pulse (frequency, $\omega_{S}$ ) were simultaneously irradiated to the sample at time $t$, the transmittance difference $(\Delta T)$ in the Stokes side induced by the stimulated Raman process can be described as follows [83].

$$
\Delta T\left(\omega_{S}, t\right) \propto \frac{N_{0}(t)-N_{1}(t)}{\left[\omega_{S}-\left(\omega_{R}-\omega_{10}\right)\right]^{2}+\left(\gamma_{10}+\gamma_{R}\right)^{2}}
$$

Here, $\omega_{10}$ corresponds to the frequency difference between $l=1$ and $l=0$ vibrational states. The observed signal has a peak at the position whose frequency is $\omega_{10}$ lower than the frequency of Raman excitation pulse $\left(\omega_{R}\right)$. Spectral bandwidth becomes the summation of the widths of vibration $\gamma_{10}$ and the Raman excitation pulse $\gamma_{R}$. Signal intensity is proportional to the difference of the population of vibrational ground and excited states. In this method, both the femtosecond time-resolution and the sufficiently high spectral resolution for Raman measurement are simultaneously satisfied. Moreover, when the population of the excited state vibration exceeds that of the ground state vibration $\left(N_{1}>N_{0}\right)$, the sign of the signal is reverted, i.e. population change of the vibrational states can be observed.

Figure 17 shows time-resolved Raman signals of $\beta$-carotene [66]. The signal at 1520 $\mathrm{cm}^{-1}$ is the $v_{1}$ mode of $\beta$-carotene in the ground $1^{1} \mathrm{Ag}^{-}\left(\mathrm{S}_{0}\right)$ state. Following photoexcitation the signal of the ground-state vibration decreases in intensity and the $v_{1}$ mode in the lowest excited singlet $2^{1} \mathrm{Ag}^{-}\left(\mathrm{S}_{1}\right)$ state appears at $1800 \mathrm{~cm}^{-1}$. The sign of the $1800 \mathrm{~cm}^{-1}$ signal is negative. This means that the $2^{1} \mathrm{Ag}^{-}\left(\mathrm{S}_{1}\right)$ state is in the vibrational excited state $(l=1$ level in Figure 16) for more than several picoseconds after photoexcitation.

Conjugation length dependence of the vibrational relaxation process in the $2^{1} \mathrm{Ag}^{-}$ 
$\left(\mathrm{S}_{1}\right)$ state was investigated by means of time-resolved stimulated Raman spectroscopy on the homologues of $\beta$-carotene that have $n$ from 7 to 15 ( $n=11$ for $\beta$-carotene) [87]. In all the homologues the decrease of the $v_{1}$ mode intensity in the ground $1^{1} \mathrm{Ag}^{-}\left(\mathrm{S}_{0}\right)$ state was observed following photoexcitation. This is due to the fact the ground state population was decreased by photoexcitation. However, $v_{1}$ mode in the $2^{1} \mathrm{Ag}^{-}\left(\mathrm{S}_{1}\right)$ state that appears around $1800 \mathrm{~cm}^{-1}$ showed the conjugation length dependence. The negative signals of the $v_{1}$ mode were observed for the homologues that have $n=11$ or less, and this $v_{1}$ mode was assigned to the vibrational excited state in the $2^{1} \mathrm{Ag}^{-}\left(\mathrm{S}_{1}\right)$ state. However, the homologues that have $n=13$ or more did not show such negative signals, so it was concluded that the $2^{1} \mathrm{Ag}^{-}\left(\mathrm{S}_{1}\right)$ state relaxed to the vibrational ground state immediately after photoexcitation in these homologues. These results show that in the shorter conjugated carotenoids vibrational relaxation of the $v_{1}$ mode is slower than several picoseconds in the $2^{1} \mathrm{Ag}^{-}\left(\mathrm{S}_{1}\right)$ state, whereas in the longer conjugated carotenoids vibrational relaxation takes place within $1 \mathrm{ps}$ after photoexcitation.

\subsection{Two-photon excitation spectroscopy}

It is desirable to control the population of initial vibrational states following photoexcitation in order to precisely investigate the vibrational relaxation process in the excited electronic state. With regard to the $1^{1} \mathrm{Bu}^{+}\left(\mathrm{S}_{2}\right)$ state of carotenoids, this relaxation process has been investigated by selective generation of vibrational ground and excited states using a wavelength-tunable excitation laser light source [88]. In the same study transient absorption spectra of the $2^{1} \mathrm{Ag}^{-}\left(\mathrm{S}_{1}\right)$ state also showed the difference depending on the excitation wavelengths, therefore, it was suggested that the vibrations induced on the carotenoid affect the relaxation process for more than several picoseconds. The experimental methods to induce molecular vibration coherently using ultrafast laser pulses have been well developed and these have now been applied to carotenoids [89]. However, it is difficult to control the population of the initial vibrational states in the $2^{1} \mathrm{Ag}^{-}\left(\mathrm{S}_{1}\right)$ state, as this electronic state is usually generated through internal conversion from the $1^{1} \mathrm{~B}_{\mathrm{u}}{ }^{+}\left(\mathrm{S}_{2}\right)$ state.

In order to solve this issue it is necessary to directly excite the $2^{1} \mathrm{Ag}^{-}\left(\mathrm{S}_{1}\right)$ state. Two-photon excitation is allowed for the transition from the ground to $2^{1} \mathrm{Ag}^{-}\left(\mathrm{S}_{1}\right)$ state. There is a report that showed the direct excitation of the vibrational excited states in the 
$2^{1} \mathrm{Ag}^{-}\left(\mathrm{S}_{1}\right)$ state using a tunable infrared excitation laser light source [90]. Walla et al observed the fluorescence from Bchl following two-photon excitation of the $2^{1} \mathrm{Ag}^{-}\left(\mathrm{S}_{1}\right)$ state of carotenoids and subsequent energy transfer in LHCII (light-harvesting pigment-protein complex in higher plants and algae). They found the increase of energy transfer efficiency from carotenoid to Bchl by the selective excitation of the vibrational excited state in the $2^{1} \mathrm{Ag}^{-}\left(\mathrm{S}_{1}\right)$ state.

The measurement of transient absorption spectra using time-resolved absorption spectroscopy is a powerful tool to reveal the vibrational relaxation processes. However, spectroscopic apparatus with sufficiently high accuracy is required for two-photon excitation, as the signals detected using this spectroscopic method are very weak. The higher accuracy of the time-resolved spectroscopy can be achieved by increasing the rate of detection of the probe signals so as to synchronize the repetition rate of the laser pulses. Using this technology an accuracy of $10^{-4}$ to $10^{-5}$ of the absorbance change $(\Delta \mathrm{A})$ is readily obtained within a short period of time of data acquisition [71].

The excitation light sources used for two-photon excitation (TPE) are in resonance with the vibrational ground (1330 nm, TPE 0-0), first excited (1230 nm, TPE 0-1), and second excited (1130 nm, TPE 0-2) states in the $2^{1} \mathrm{Ag}^{-}\left(\mathrm{S}_{1}\right)$ state as shown in Figure 15. These excitation light sources are not in resonance with the $1^{1} \mathrm{~B}_{\mathrm{u}}{ }^{+}\left(\mathrm{S}_{2}\right)$ state, but nonlinear optical effect between the excitation and probe pulses induce a large transient signal at the delay time of 0.0 ps (time-origin) [53]. However, this nonlinear signal immediately disappears and the signals detected after $0.5 \mathrm{ps}$ following excitation are assignable to those of the $2^{1} \mathrm{Ag}^{-}\left(\mathrm{S}_{1}\right)$ state directly generated by two-photon excitation. Figure 18 shows the transient absorption spectra of $\beta$-carotene recorded at 5 ps after two-photon excitation [91]. Apparent differences can be seen between the spectra of TPE 0-0 excitation and the other excitations (TPE 0-1 and TPE 0-2). In the case of TPE 0-0 excitation the maximum absorbance of transient absorption appears at $2.23 \mathrm{eV}$ (556 $\mathrm{nm})$, whereas the corresponding maxima shift to lower in energy at $2.21 \mathrm{eV}(561 \mathrm{~nm})$ for other excitations. This shift can be explained by the difference of the states of $v_{1}$ mode between TPE 0-0 and other excitations. Moreover, the signal due to vibrational relaxation shows an increase until 1 ps after excitation in the case of TPE 0-2 excitation. However, in the cases of TPE 0-0 and TPE 0-1 excitations an increase of the signal due to vibrational relaxation is not observed. As the consequence, the vibrational relaxation processes in the $2^{1} \mathrm{Ag}^{-}\left(\mathrm{S}_{1}\right)$ state can be summarized as follows. 
(1) When the vibrational ground state $(l=0)$ in the $2^{1} \mathrm{Ag}^{-}\left(\mathrm{S}_{1}\right)$ state is excited, transient absorption from the $l=0$ state is observed. (2) When the vibrationally excited $l$ $=1$ state in the $2^{1} \mathrm{Ag}^{-}\left(\mathrm{S}_{1}\right)$ state is excited, the excited electronic state stays in the $l=1$ level of the $v_{1}$ mode for more than $5 \mathrm{ps}$. Therefore, a signal change due to vibrational relaxation cannot be observed. The maximum of transient absorption shows the low energy shift reflecting the effect of strong vibronic coupling as mentioned above. (3) When the vibrationally excited $l=2$ state in the $2^{1} \mathrm{Ag}^{-}\left(\mathrm{S}_{1}\right)$ state is excited, vibrational relaxation to the $l=1$ level of the $v_{1}$ mode takes place within 1 ps after excitation. However, the excited state then stays in the $l=1$ state for more than $5 \mathrm{ps}$, the transient absorption spectrum that shifted lower in energy can be observed.

Both the time-resolved Raman and two-photon excitation spectroscopies clarified that vibrationally excited $l=1$ state of the $v_{1}$ mode in the $2^{1} \mathrm{Ag}^{-}\left(\mathrm{S}_{1}\right)$ state of $\beta$-carotene has an amazingly long lifetime of more than several picoseconds. This finding suggests that the vibrational excited state in the excited electronic state of carotenoids may have an important role in the light-harvesting process of photosynthesis.

\section{Coherent Spectroscopy on Carotenoids}

Coherent spectroscopy has been developed using gaseous samples such as iodine [92-95], mainly because information on both the electronic and vibrational states had already been obtained and understood for these relatively simple, gaseous samples. In addition, the period of vibrational motions of these samples were longer than the laser pulses to be used for the measurements and, therefore, the discrimination of the signals characteristic of coherent spectroscopy was relatively straightforward for such samples. Subsequently, owing to the development of new techniques as well as improved laser light sources, coherent spectroscopy has been able to be applied to more complicated systems such as liquids, inorganic solids and proteins, etc. Observing the coherent component of the signals makes it possible to obtain unified information on the dynamics of both vibrational and electronic states, interaction between these states and the effect of the surrounding environment on the materials. Moreover, controlling the shape and interval as well as the phase of incident laser pulses can regulate the efficiencies of photochemical reactions, i.e. reaction pathways. 
Coherent signals appear due to quantum mechanical interference between the states and so reflect the magnitude of the coupling between the states. In what follows, coherent signals that have been observed for carotenoids are surveyed. The readers should consult the cited literature for the details of the physical background to further understand coherent signals $[96,97]$.

\subsection{Image of $n$-Wave Mixing Signal}

Figure 19 shows an example of four-wave mixing (FWM) and six-wave mixing (SWM) signals from $\beta$-carotene [89]. In general $n$-wave mixing measurement is performed with an optical configuration illustrated in Figure 20(a). Namely, laser light is split to three using a beam splitter and two of them are independently guided toward translational stages in order to induce time delays among three laser pulses. If these three laser pulses are well focused on a single spot of the sample, FWM signals can be observed. This type of optical configuration, where three laser pulses are irradiated to the sample from three distinct directions, is called the BOXCARS configuration.

Three intense laser spots surrounding the central triangle in Figure 19 are the excitation laser lights transmitted through the sample. When three excitation laser pulses simultaneously reach to the sample (zero time delays), FWM and SWM signals are generated in the area surrounding the transmitted excitation laser light. As illustrated in Figure 20(b), if the wave-vectors of excitation and signal lights are defined, respectively, as $k_{i}(i=1,2,3)$ and $k_{S}, \mathrm{FWM}$ signals appear at the direction that satisfies the relation of $k_{S}= \pm k_{1} \pm k_{2} \pm k_{3}$ and $k_{S}=2 k_{i}-k_{j}(j=1,2,3$ and $j \neq i)$. SWM signals that are pointed out with arrows in Figure 19 appear following additional interaction with two more excitation laser pulses ( 5 times interaction all together), for example as $k_{S}=-2 k_{1}+2 k_{2}+k_{3}$. It should be noted here that FWM and SWM signals are strong enough for carotenoids to be identified with the naked eye. This means that carotenoids are suitable molecules for investigation of their nonlinear optical responses. The information concerning the coherence can be obtained by investigating the time evolution of the FWM signals.

In the next section, coherent spectroscopy based on the measurements of FWM signals is described. The wording of four-wave means that one signal wave is obtained with the irradiation of three incident light waves, i.e. four light waves all together are 
closely related to each other in order to generate FWM signals.

\subsection{Four-Wave Mixing (FWM) Signal}

FWM signals in carotenoids have been reported for $\beta$-carotene and its homologues, lycopene, astaxanthin and spheroidene [89, 98-109]. As an example, the results of $\beta$-carotene are given here. Figure 21(a) shows the time evolution of a FWM signal in $\beta$-carotene, which is obtained by observing a single spot shown in Figure 19. In this example, the abscissa gives the time interval $T$ between pulse 2 and pulse 3 (see Figure 20(c)). The time interval $\tau$ between pulse 1 and pulse 2 was set to be zero. The FWM signal that is measured under this condition is frequently called as a transient grating (TG) signal. The intense signal that appears around time origin in Figure 21(a) is assigned as a coherent spike. Following this spike signal, coherent vibration signal with the very fast period of oscillation in about 20 - 30 fs can be observed on top of the slowly decaying background with a lifetime about 5 ps.

The origin of this coherent vibration can be clarified if the TG signal in Figure 21(b) is Fourier transformed. It can be readily understood, based on the comparison with the Raman spectrum of $\beta$-carotene shown in Figure 22(b), that the peaks obtained by the Fourier transformation of the coherent vibration (Figure 22(a)) show good coincidence with those of the Raman spectrum of $\beta$-carotene. Namely, the peaks that appeared at $v_{1}$ $=1522 \mathrm{~cm}^{-1}$ and $v_{2}=1157 \mathrm{~cm}^{-1}$, respectively, are attributed to the totally symmetric vibration of $\mathrm{C}=\mathrm{C}$ and $\mathrm{C}-\mathrm{C}$ stretchings and the peak that appeared at $\mathrm{v}_{3}=1007 \mathrm{~cm}^{-1}$ is attributed to the in-plane rocking vibration of methyl groups. These vibrational modes appeared because all the $\beta$-carotene molecules under inspection start to vibrate in phase, i.e. coherently, following the impulsive excitation with ultrashort laser pulses. The stretching vibrations of carotenoids usually appear in the $1000-1500 \mathrm{~cm}^{-1}$ frequency domain. These frequencies correspond to $30-20 \mathrm{fs}$ in the time domain, if the frequency to time domain conversion is performed. Therefore, coherent vibration can be induced in carotenoids, if we use the sub-20 fs ultrashort laser pulses for the FWM experiment. As illustrated in Figure 23, the first and second laser pulses generate a coherent state in the vibrational ground ( $g$ and $\left.g^{\prime}\right)$ and excited ( $e$ and $\left.e^{\prime}\right)$ states and as a consequence real-time observation of vibrational wave-packets becomes possible.

The most important information that is obtained by the measurement of FWM 
signals is the coupling between carotenoids and their surrounding environment. This information is reflected in spectral density (see Figure 22(c)). It is known that there are couplings with slow vibration of $100 \mathrm{fs}\left(\sim 300 \mathrm{~cm}^{-1}\right)$ or less in organic solvents [89, 103, 105]. Obtaining the spectral density, various optical responses including absorption and fluorescence spectra can be calculated and, therefore, the precise discussion on the experimental data based on theoretical models becomes feasible [96]. One of those examples, Figure 21(c), shows the results of the theoretical calculation for the FWM signal. The experimental result is nicely in agreement with the theoretical calculation. As shown here spectral density includes meaningful information, however, there are few reports on carotenoids bound to pigment-protein complexes [110]. On the other hand, many studies have already been performed on the coherent vibrations that directly reflect the effect of coupling with surrounding environment in Bchl [111-118]. Revealing the correlation among these coherent vibrations as well as the role of coherent vibration in excitation energy transfer will be rewarding challenges for the future.

Coherent vibrations are also observed in the electronic excited state of a carotenoid. Motzkus et al. were able to observe the coherent vibration in the $\mathrm{S}_{1}$ state of $\beta$-carotene with $20 \mathrm{fs}$ temporal and $10 \mathrm{~cm}^{-1}$ spectral resolutions [99, 100, 102]. They introduced pre-pump pulse that excites $\beta$-carotene to its $\mathrm{S}_{2}$ state for FWM measurement (pump-FWM) to produce a populated $\mathrm{S}_{1}$ state via internal conversion from the $\mathrm{S}_{2}$ state. They concluded that coherence of molecular vibration is not conserved during the process of $S_{2} \rightarrow S_{1}$ internal conversion [99]. They also showed that the lifetime of coherent vibration in the $\mathrm{S}_{1}$ state is an order of magnitude smaller than that in the ground state for all the vibrational modes.

We have so far described the coherence between vibrational states. If the vibrational states shown in Figure 23 are exchanged to electronic states, it can be readily imagined that coherence between electronic states should also be detectable. However, the observation of electronic coherence itself is one of the hottest topics in the research field of ultrafast spectroscopy and, as yet, there are almost no reports on the electronic coherence in carotenoids. In following section some relevant reports are highlighted. Very long-lived ( $>660 \mathrm{fs}$ ) coherence among electronic excited states was found in Fenna-Matthew-Olson (FMO) Bchl complex isolated from a green sulfur bacterium Chlorobium tepidum [119]. This observation suggests that the excitation energy-transfer 
among Bchls in FMO complex cannot be simply explained by the classical picture that shows the hopping of excitation energy between each of the Bchl molecules, rather it should be interpreted as the superposition of quantum mechanical states. Electronic coherence was also found between $\mathrm{H}$ Bphe and accessory Bchl molecules in the RC from the purple photosynthetic bacterium Rba. sphaeroides [120]. The remarkable finding in this report is that the decoherence time (440 fs) is much longer than the excitation energy-transfer time (250 fs) from $\mathrm{H}$ to accessary Bchl. This result suggests a possibility that electronic coherence may have some relevancy in the excitation energy-transfer process. Moreover, coherent energy-transfer was found within the conjugated system in $\pi$-conjugated polymer MEH-PPV [121]. One of the big challenges remaining for photosynthesis research is to obtain detailed information on the coherence within and between carotenoids and that between carotenoid and Bchl.

\subsection{Coherent Anti-Stokes Raman Scattering (CARS)}

CARS spectroscopy is a similar spectroscopic technique to FWM spectroscopy in the sense that both observe the third-order nonlinear optical response by irradiating three independent incident radiations to the sample. The main differences between them are that CARS uses spectrally broad light for the second pulse (called a Stokes light) and that CARS signals appear at the higher energy side of the excitation light (anti-Stokes side).

Hamaguchi et al. were successfully able to apply the CARS technique to $\beta$-carotene with 1.2 ps time-resolution [122]. Introducing vibrationally hot ground state $\mathrm{S}_{0} *$ in the process of $\mathrm{S}_{1} \rightarrow \mathrm{S}_{0}$ internal conversion, these authors have estimated the relaxation time constants of $\mathrm{S}_{1} \rightarrow \mathrm{S}_{0} *$ and $\mathrm{S}_{0} * \rightarrow \mathrm{S}_{0}$, respectively, to be 7 ps and 11 ps. The results from this study show good agreement with those time constants determined independently using femtosecond time-resolved Raman spectroscopy [66, 68]. Siebert et al. applied pump-CARS measurement to $\beta$-carotene, in which the pre-pump laser pulse was introduced before starting the CARS measurement and reported the relationship between the relaxation of excitation energy and molecular vibrations [123-125]. According to the series of studies, they concluded that the $\mathrm{C}=\mathrm{C}$ stretching vibration $\left(1524 \mathrm{~cm}^{-1}\right)$ has the largest contribution as the accepting mode for excitation energy, and the other modes have a similar but lower contribution in the order; C-C stretching (1157 
$\left.\mathrm{cm}^{-1}\right), \mathrm{C}-\mathrm{CH}_{3}$ rocking $\left(1004 \mathrm{~cm}^{-1}\right), \mathrm{C}=\mathrm{C}-\mathrm{C}$ bending $\left(872 \mathrm{~cm}^{-1}\right)$, and $\mathrm{C}-\mathrm{H}$ bending $(1269$ $\left.\mathrm{cm}^{-1}\right)$.

\subsection{Pump-Probe Spectroscopy}

The reasons why FWM spectroscopy is used to detect coherent signals are; (1) the FWM signals appear not on the same optical axis of the excitation light sources, background-free signals can be obtained, (2) changing the time intervals between each of three excitation laser pulses makes it possible to select the required response functions. However, it is known that coherent molecular vibrations can be detected without using the FWM technique. One of the most representative ones is to use pump-probe spectroscopy with an extremely ultra-short laser pulse (sub-10 fs). With this spectroscopic technique, as the three laser pulses interact with each other to generate the signals, so similar information to that from FWM spectroscopy can also be obtained. Cerullo et al. have observed the coherent vibrations in the ground state of $\beta$-carotene, okenone, and rhodopin-glucoside in a wide spectral regime [126-129]. Most of the previous reports using pump-probe spectroscopy have focused their attention on the population dynamics and so the number of publications that pay attention to the coherence of the system are still very restricted. Nevertheless, some interesting phenomena have already been described; (1) the coherent vibration changes its phase with the amount of $\pi$ nearby the isosbestic point that has appeared because of the competition of both bleaching of the ground state absorption and the appearance of $\mathrm{S}_{\mathrm{n}}$ $\leftarrow \mathrm{S}_{1}$ transient absorption and, (2), the phase of coherent vibration changes continuously at time due to the effect of frequency chirp of probe light [129].

\subsection{Coherent Control}

Recently, expanding the utility of coherent spectroscopy, motivation on the research to control the photophysical and photochemical reaction pathways is accelerated. Namely, when the ensemble of molecules is moving along some reaction coordinates, it can be guided to the selective reaction pathway suppressing simultaneously unnecessary pathways by the use of ultra-short laser pulses.

As already mentioned above, sub-20 fs laser pulses are required to excite coherently 
the molecular vibrations of carotenoids. Because of the uncertainty principle in quantum mechanics, such an ultra-short laser pulse is spectrally broad. As a consequence, excitation using ultra-short laser pulses induces various coherent modes of molecular vibration (see Figure 23). An optical pulse-shaping device such as a space-light modulator can generate periodic laser light pulses called pulse trains. If the period of these pulse trains, for example, were adjusted to match the period of a specific molecular vibrational mode, then only the specific coherent vibrational mode can be excited and the other coherent modes can completely be suppressed. The $\mathrm{C}-\mathrm{CH}_{3}$ in-plain rocking, $\mathrm{C}-\mathrm{C}$ stretching, and $\mathrm{C}=\mathrm{C}$ stretching modes of $\beta$-carotene in cyclohexane, respectively, have the frequencies of $v_{3}=1004 \mathrm{~cm}^{-1}, v_{2}=1157 \mathrm{~cm}^{-1}$, and $v_{1}=1524 \mathrm{~cm}^{-1}$. These frequencies, when converted to time domain (period), then become 33.2 fs, 28.8 fs, and 21.9 fs, respectively. Hauer et al. were successful to selectively excite coherent vibrations corresponding to either of these vibrational modes using such shaped pulse trains [130]. By specifically adopting a resonance excitation condition, the selectively excited coherent vibrational mode was enhanced in intensity 5.7 times larger than that without using the pulse-shaping technique. Konradi et al. confirmed the validity of the selective mode excitation almost at the same time [131].

Motzkus et al. were successfully able to quantum control the excitation energy-transfer (ET) efficiency from carotenoid to Bchl in the LH2 complex from a purple photosynthetic bacterium Rbl. acidophilus [132-134]. They regulated the phase at each wavelengths of a femtosecond laser pulse using a space-light modulator based on the application of evolutional algorithms by monitoring the ET efficiency as well as the rate of internal conversion (IC) of carotenoid. It was shown that $30 \%$ of the ratio of IC/ET can be controlled and that ET and IC states can be switched by changing the phase of $\pi$ for excitation light pulse. This is indeed a striking finding because complicated photosynthetic light-harvesting reactions have proved to be controllable. In the first publication downward regulation of the ET from carotenoid to Bchl was reported and the authors suggested that in principle upward regulation might become possible. Due to the improvement of evolutional algorithms, this idea was subsequently proved to be true and the authors were able to enhance the efficiencies of ET and IC independently. Periodic pulse trains are used in order to make feasible coherent control experiments. In carotenoids, it is proposed that the key factor is to synchronise the period of the pulse trains to that of the coherent vibrations. 


\section{Artificial Light-Harvesting Systems}

In order to make the practical usage of artificial light-harvesting antenna systems feasible it should be remembered that the type of light that is to be harvested needs to appropriately reflect the energy necessary to facilitate the chemistry required for solar fuel production. The light-harvesting system should not only be efficient but also have a sufficient energy cross section. In this regard, carotenoids can be important components in the construction of bio-inspired artificial antenna systems. In this section some of the experimental techniques that are working towards this aim are introduced.

\subsection{Reconstitution of the Light-Harvesting Antenna from Purple Photosynthetic Bacteria}

Reconstitution of photosynthetic pigment protein complexes has been proved to be a powerful technique with which not only to study function of carotenoids but also towards constructing bio-mimetic artificial light-harvesting antenna [135]. In this regard using the LH1 complex is advantageous since LH1 complexes can be fully reconstituted in vitro from its constituent parts, i.e., $\mathrm{LH} 1-\alpha$ and $\mathrm{LH} 1-\beta$ polypeptides and the pigments (Bchl $a$ and carotenoid) [136-144]. With reconstitution it is possible to study the effects of adding a series of carotenoids where such factors as $n$ can be varied in a systematic way. As the photochemical properties of carotenoids change in a graded way depending on the number of conjugated double bonds, such studies can provide key insight into the mechanism of carotenoid function in situ.

Reconstitution of the LH1 complex from the purple photosynthetic bacterium Rsp. rubrum S1 was performed with a range of carotenoid molecules having different $n$ [145]. Since some of the added carotenoids tended to aggregate and then to bind non-specifically to the reconstituted LH1 complexes [146] a further purification step, using a sucrose density gradient centrifugation, was introduced to improve purity of the final reconstituted sample. The measured absorption, fluorescence-excitation, and Stark absorption spectra of the LH1 complex reconstituted with spirilloxanthin were identical with those obtained with the native, spirilloxanthin-containing, LH1-RC complex of 
Rsp. rubrum S1. This shows that the electrostatic environments surrounding the carotenoid and Bchl $a$ molecules in both of these LH1 complexes were essentially the same. This was further tested by measuring the polarization angle dependence of Stark absorption spectra [147]. Stark absorption spectra of spirilloxanthin bound to both the native and reconstituted LH1 complexes were compared in different polarization angles against the external electric field. From the polarization angle dependence of the Stark absorption spectra, two angles could be determined in reference to the direction of transition dipole moment of spirilloxanthin: one is the change in polarizability upon photoexcitation $(\Delta \alpha), \theta \Delta \alpha$ and the other is the change in static dipole moment upon photoexcitation $(\Delta \mu), \theta \Delta \mu$. Despite the symmetric molecular structure of all-trans spirilloxanthin, its Stark absorption spectra show pronounced values of $\Delta \mu$. This large $\Delta \mu$ value essentially caused by the effect of induced dipole moment through $\Delta \alpha$ both in the cases for native and reconstituted LH1 complexes. However, slightly different values of $\theta \Delta \alpha$, and $\theta \Delta \mu$ observed for the native LH1 complex suggest that spirilloxanthin is asymmetrically distorted when bound to the native LH1 complex and gives rise to intrinsic $\Delta \mu$ value.

In the LH1 complexes reconstituted with either rhodopin or spheroidene, however, the wavelength maximum at the $\mathrm{Bchl} a \mathrm{Q}_{\mathrm{y}}$ absorption band was slightly different to that of the native LH1 complexes. These differences in the transition energy of the Bchl $a$ Qy absorption band can be explained using the values of the nonlinear optical parameters of this absorption band, i.e., the polarizability change $\operatorname{Tr}(\Delta \alpha)$ and the static dipole-moment change $|\Delta \mu|$ upon photoexcitation, as determined using Stark spectroscopy. The local electric field around the Bchl $a$ in the native LH1 complex was determined to be $\sim 3.0 \times$ $10^{6} \mathrm{~V} / \mathrm{cm}$. Furthermore, on the basis of the values of the nonlinear optical parameters of the carotenoids in the reconstituted LH1 complexes, it was possible to suggest that the conformations of carotenoids, anhydrorhodovibrin and spheroidene, in the LH1 complex were similar to that of rhodopin glucoside in crystal structure of the LH2 complex from Rbl. acidophilus 10050.

In the case of the rhodopin complex, small anomalies were observed in the absorption maximum of the $\mathrm{Q}_{\mathrm{y}}$ band of $\mathrm{Bchl} a$ (Bchl $a \mathrm{Q}_{\mathrm{y}}$ ) and the singlet-singlet energy-transfer efficiency of carotenoid to Bchl $a$ within the LH1 carotenoid complex. The reason for these anomalies can probably be ascribed to the hydroxy group in rhodopin. To investigate this further, reassociation experiments of the LH1 complex 
with hydroxy-protected rhodopin, all-trans-3,4-dihydroanhydrorhodovibrin, were conducted [148]. In such a case a normal absorption maximum $(883 \mathrm{~nm})$ of the Bchl $a$ $\mathrm{Q}_{\mathrm{y}}$ band in the LH1-1 complex was observed. The energy-transfer efficiency of carotenoid to Bchl $a$ was found to be $53 \%$, giving a good correlation with the other carotenoids studied.

\subsection{Photophysical Properties of Molecular Aggregates of Carotenoids}

As explained above, in natural photosynthetic systems, one of the roles of carotenoids is light harvesting: carotenoids absorb light energy and transfer it to chlorophylls [6, 76, 149]. Fucoxanthin is a carotenoid bound to the major photosynthetic antenna of brown algae, called the fucoxanthin-chlorophyll $a / c$ binding protein (FCP) as has already been shown above [33, 35]. The major absorption band of fucoxanthin shows a large bathochromic shift $(\sim 50 \mathrm{~nm})$ when it is bound to the FCP. Consequently, the fucoxanthin in FCP can efficiently absorb the blue-green region of the solar spectrum, which is the only part of that spectrum that is available in their habitat several meters below the surface of the sea. The large bathochromic shift cannot be explained simply by the polarizability of fucoxanthin and the environment of this molecule in FCP; consequently it is necessary to investigate whether dipole-dipole interactions from adjacent fucoxanthin molecules are also important. However, the key factors that could determine such dipole-dipole interactions, such as distance and orientation between fucoxanthin molecules in the FCP, have not yet been clarified. Therefore, an artificial model system was created in which the intermolecular interactions can be observed and controlled in order to try to reproduce the large bathochromic shift [150].

Molecular aggregates are one of the situations where interactions are present between carotenoid molecules. It is known that excitonic interaction occurs between aggregated molecules and their associations in these aggregates are classified into $\mathrm{H}$ (card pack) type and J- (head-to-tail) type, which show a hypsochromic (blue) shift and a bathochromic (red) shift of the absorption band, respectively [151, 152]. Further, such electronic interactions of two individual molecules are clearly observed even if they are separated by as much as $12 \mathrm{~nm}$ [153]. Self-aggregation of carotenoid often takes place in aqueous organic solutions [154-160]. Carotenoid aggregates have also been observed 
in thin solid films [161-163] and in composite molecules [164, 165]. Nevertheless, the self-assembly of fucoxanthin has not yet been reported. Therefore, experiments were designed to try and observe fucoxanthin aggregates in mesopores materials.

Mesoporous silica is a form of silicon dioxide that has nano-scale pores [166]. Itoh et al. reported that the mesopore spaces of FSM (folded-sheet mesoporous material) allowed nano-scale interactions between adsorbed chlorophyll $a$ molecules [167-169]. Therefore, experiments were attempted to adsorb fucoxanthin molecules into the mesopores of a silica gel called CARiACT and to observe the change of optical properties of fucoxanthin that this produced [150]. Fucoxanthin in the pores of CARiACT showed spectra that suggested the formation of $\mathrm{H}$ - or J-aggregates. The form of the aggregate could be controlled by adjusting the ratio of fucoxanthin to CARiACT. The exciton chirality of $\mathrm{H}$-aggregated fucoxanthin was detected by circular dichroism spectroscopy. Based on the point-dipole approximation, the molecular arrangement of $\mathrm{H}$-aggregated fucoxanthin in the pores of CARiACT was able to be determined. Thus, aggregates of fucoxanthin can be observed and controlled, a finding that has never been reported for self-association case in solution phase. The conjugate of carotenoids and mesoporous materials is a promising tool with which to control the molecular arrangement and hence the intermolecular interactions of carotenoids in aggregates.

\subsection{Dyad and Triad Systems Using Carotenoids}

One of possible approaches when constructing an artificial photosynthesis mimic is to use synthetic chromophores that are related to the natural pigments but are more stable and are bound covalently. The overall structures of artificial photosynthesis constructs would somewhat resemble the natural photosynthetic systems in which antenna pigments convert solar photons to excitation energy, regulate the flow of excitation energy to RCs, and suppress the production of reactive oxygen species (photoprotection). RCs generate transmembrane redox potential, which is used for both promotion of redox chemistry and conversion to proton motive force. Dyads and triads systems containing carotenoids have proved to be a powerful tool towards achieving this objective [170].

Gust, Moore, and Moore group designed and synthesized a series of carotenoid-porphyrin (C-P) dyads [171, 172] (see Figure 24 for the chemical structures). 
Some of these showed hole transfer from the oxidized porphyrin $\left(\mathrm{P} \bullet^{+}\right)$to the carotenoid to yield the $\bullet^{+}-\mathrm{P}$ species [173]. The synthesis of a carotenoid-porphyrin-quinone (C-P-Q) triad system was performed based on the idea of combining this result with the photoinduced formation of $\mathrm{P} \bullet^{+}-\mathrm{Q} \bullet-[174,175]$. The photogenerated excited singlet state of the porphyrin in the triad $\left(\mathrm{C}-{ }^{1} \mathrm{P}-\mathrm{Q}\right)$ is proved to generate the $\mathrm{C}-\mathrm{P} \bullet{ }^{+}-\mathrm{Q} \bullet \bullet^{-}$charge separated state by electron transfer using transient spectroscopic studies. A second electron transfer from carotenoid to the porphyrin radical cation follows, which produces the final charge separated state $\left(\mathrm{C}^{+}-\mathrm{P}-\mathrm{Q} \bullet^{-}\right)$. This final state has a relatively long lifetime, $170 \mathrm{~ns}$ in dichloromethane and $2.5 \mu \mathrm{s}$ in electrolyte-saturated solvent [174]. These artificial photosynthesis triad systems experimentally demonstrate one of the fundamental concepts of natural photosynthesis. Namely, a series of short-range, fast, efficient electron transport can result in the formation of long-lived charge separated states. The theoretical background for this strategy was provided by Marcus and others [176]. The concepts demonstrated by the original triad proved to be universal and have been extended to more complex multiple component systems [177-182]. Among them the prototypical carotenoid(C)-porphyrin(P)-fullerene $\left(\mathrm{C}_{60}\right)$ type of structures have been the most studied (see Figure 24 for chemical structure of C-P-C60 triad). Their energy and charge-transfer processes were characterized from photoexcitation to fluorescence $[177,182,183]$. Time-resolved optical measurements have also been performed for related light-harvesting systems [183, 184]. A number of theoretical analyses to investigate the photoinduced charge-separation dynamics of C-P-C60 system have been reported [185-191]. However, the detailed mechanisms remain to be addressed.

\section{Conclusion}

Carotenoids are fascinating molecules with remarkable photophysical properties [7]. Their functions can be tuned and regulated by proteins and they participate in a wide variety of reactions, principally in photosynthesis, that exploit their chemical and photophysical potentials. Though a lot of detail is known about how carotenoids participate in these functions there is still a lot more detail to be uncovered. Now is a particularly exciting time to be involved in carotenoid research. It has been particularly 
notable that as the advancement in the technology underpinning the physical methods that have been applied to studying carotenoids has increased, this then correlates directly with our increasing knowledge of the way in which carotenoids function in photosynthesis. One can expect this trend to continue and areas can be highlighted where it is expected that these developments will really help to understand the molecular mechanisms by which carotenoids discharge their photosynthetic functions. Examples are the further application of advanced 2D coherent time-resolved spectroscopies [192, 193] and time-resolved stimulated Raman spectroscopy [83-86]. These methods should be able to help resolve the ongoing problems of understanding the pattern of carotenoid excited singlet states and their involvement in light-harvesting. Further application of X-ray crystallography [194] with, hopefully, the provision of structural information on LHCII in both the quenched and non-quenched states to help reveal the role carotenoids play in NPQ (non-photochemical quenching). New intense $\mathrm{X}$-ray sources such as those produced by free electron lasers open the door to both time-resolved structural studies and ultimately the ability to determine the structures of individual molecules [10, 195-197]. This will then allow such processes as carotenoid isomerization to be visualized, which has been a long held dream of workers in this area.

Clarifying all the detailed mechanisms of carotenoids in their electronic excited states may surely open the doors to construct 'bio-inspired' artificial light-harvesting mimetic systems. The authors do not think that natural pigment-protein complexes can directly be utilized for a final device of artificial photosynthesis. In this regard the use of the word 'bio-inspired' is appropriate as the intention is not simply to mimic Nature per se. The eventual aim is to construct robust pigment systems that should be used along the design principles set out in the previous sections of this review. To aid in this, lessons should always be learned by comparing and contrasting the different light-harvesting systems and determining what properties lend themselves towards providing a suitable solution. Conversely, it should be noted what are the common principles, amongst the various systems, that can be exploited in order to help fully design better artificial systems? It will be the major challenge in the next generation to comprehensively interpret the experimental data obtained using the sophisticated spectroscopies explained in this review. Support from theoretical studies is indispensable in order to verify the results of ultrafast spectroscopies. Owing to the 
rapid improvement in the data processing power of computer systems, more accurate theoretical calculations that include the effect of electronic couplings (compensation for the effect of interaction among many electrons) have become possible for molecules with the larger number $n$ of conjugated $\mathrm{C}=\mathrm{C}$ bonds than the classical works by Tavan and Schulten [47, 48]. In the reports of Tavan and Schulten the largest number of $n$ was restricted to 8 . However, Kurashige et al. were successful to extend the number to $n=$ 14 [198]. Especially in recent years the algorithm of quantum chemical calculations has been well established. As a consequence theoretical calculations that include time-dependent perturbation, as well as complicated electronic couplings, have been reported [198-200]. The calculation methods of the exact energies of complex molecules in the electronic excited-states have not yet been established so far. It is hoped that precise calculations on the electronic excited-states of carotenoids will be possible in near future for usage in artificial photosynthesis.

\section{Acknowledgements}

The authors thank JST, JSPS, MEXT, HFSP, and BBSRC for financial support. HH thanks Scientific Research on Innovative Areas "All Nippon Artificial Photosynthesis Project for Living Earth (AnApple)" (No. 24107002H) from the Japan Society for the Promotion of Science (JSPS) for financial support. This work was supported by JSPS KAKENHI Grant-in-Aid for JSPS fellows (No. 254691) to CU. YS thanks Grant-in-Aid for Scientific Research (No. 23750196) from JSPS for financial support. RJC and ATG wish to gratefully thank the BBSRC and Photosynthetic Antenna Research Center (PARC), an Energy Frontier Research Center funded by the DOE, Office of Science, Office of Basic Energy Sciences under Award Number DE-SC 0001035 for financial support.

\section{References}

[1] G. Britton, S. Liaaen-Jensen, H. Pfander, Carotenoids, Vol. 3: Biosynthesis and Metabolism, Birkhäuser, Basel, Boston, Berlin, 1998. 
[2] S.W. Jeffrey, R.F.C. Mantoura, S.W. Wright, Phytoplankton Pigments in Oceanography; Guidelines to Modern Methods (2nd ed.), UNESCO, Paris, 2005.

[3] B.R. Green, W.W. Parson, Light-Harvesting Antennas in Photosynthesis, Kluwer Academic Publishers, Dordrecht, Boston, London, 2003.

[4] P. Fromme, Photosynthetic Protein Complexes; A Structural Approach, Wiley-Blackwell, Weinheim, 2008.

[5] A. Young, G. Britton, Carotenoids in Photosynthesis, Chapman \& Hall, London, 1993.

[6] H.A. Frank, R.J. Cogdell, Carotenoids in photosynthesis, Photochem Photobiol, 63 (1996) 257-264. [DOI: 10.1111/j.1751-1097.1996.tb03022.x]

[7] H.A. Frank, A.J. Young, G. Britton, R.J. Cogdell, The Photochemistry of Carotenoids, in: Govindjee (Ed.) Advances in Photosynthesis, Kluwer Academic Publishers, Dordrecht, 1999.

[8] G. Centi, S. Perathoner, Towards solar fuels from water and $\mathrm{CO}_{2}$, ChemSusChem, 3 (2010) 195-208. [DOI: 10.1002/cssc.200900289]

[9] Y. Umena, K. Kawakami, J.-R. Shen, N. Kamiya, Crystal structure of oxygen-evolving photosystem II at a resolution of $1.9 \AA$, Nature, 473 (2011) 55-60. [DOI: 10.1038/nature09913]

[10] M. Suga, F. Akita, K. Hirata, G. Ueno, H. Murakami, Y. Nakajima, T. Shimizu, K. Yamashita, M. Yamamoto, H. Ago, J.R. Shen, Native structure of photosystem II at 1.95 $\AA$ resolution viewed by femtosecond X-ray pulses, Nature, 517 (2015) 99-103. [DOI: 10.1038/nature13991]

[11] H. Inoue, T. Shimada, Y. Kou, Y. Nabetani, D. Masui, S. Takagi, H. Tachibana, The water oxidation bottleneck in artificial photosynthesis: how can we get through it? An alternative route involving a two-electron process, ChemSusChem, 4 (2011) 173-179. [DOI: $10.1002 /$ cssc.201000385]

[12] F. Kuttassery, S. Mathew, D. Yamamoto, S. Onuki, Y. Nabetani, H. Tachibana, H. Inoue, Artificial Photosynthesis Sensitized by Metal Complexes: Utilization of a Ubiquitous Element, Electrochemistry, 82 (2014) 475-485. [DOI: 10.5796/electrochemistry.82.475]

[13] R.K. Clayton, Photosynthesis: Physical mechanisms and chemical patterns, Cambridge University Press, Cambridge, 1980.

[14] R.E. Blankenship, M.T. Madigan, C.E. Bauer, Anoxygenic Photosynthetic Bacteria, 
Kluwer Academic Publishers, Dordrecht, Boston, London, 1995.

[15] C.N. Hunter, F. Daldal, M.C. Thurnauer, J.T. Beatty, The Purple Photosynthetic Bacteria, Springer, Dordrecht, 2009.

[16] R.E. Fenna, B.W. Matthews, Chlorophyll arrangement in a bacteriochlorophyll protein from Chlorobium limicola, Nature, 258 (1975) 573-577. [10.1038/258573a0]

[17] J. Deisenhofer, O. Epp, K. Miki, R. Huber, H. Michel, Structure of the Protein Subunits in the Photosynthetic Reaction Center of Rhodopseudomonas-Viridis at $3 \AA$ Resolution, Nature, 318 (1985) 618-624. [DOI: 10.1038/318618a0]

[18] P. Fromme, P. Jordan, N. Krauss, Structure of photosystem I, Biochim. Biophys. Acta, 1507 (2001) 5-31. [DOI:10.1016/S0005-2728(01)00195-5]

[19] Z. Liu, H. Yan, K. Wang, T. Kuang, J. Zhang, L. Gui, X. An, W. Chang, Crystal structure of spinach major light-harvesting complex at $2.72 \AA$ resolution, Nature, 428 (2004) 287-292. [DOI: 10.1038/nature02373]

[20] G. Mcdermott, S.M. Prince, A.A. Freer, A.M. Hawthornthwaitelawless, M.Z. Papiz, R.J. Cogdell, N.W. Isaacs, Crystal-Structure of an Integral Membrane Light-Harvesting Complex from Photosynthetic Bacteria, Nature, 374 (1995) 517-521. [DOI: $10.1038 / 374517 \mathrm{a} 0]$

[21] J. Koepke, X. Hu, C. Muenke, K. Schulten, H. Michel, The crystal structure of the light-harvesting complex II (B800-850) from Rhodospirillum molischianum, Structure, 4 (1996) 581-597. [DOI:10.1016/S0969-2126(96)00063-9]

[22] A.W. Roszak, T.D. Howard, J. Southall, A.T. Gardiner, C.J. Law, N.W. Isaacs, R.J. Cogdell, Crystal structure of the RC-LH1 core complex from Rhodopseudomonas palustris, Science, 302 (2003) 1969-1972. [DOI: 10.1126/science.1088892]

[23] P. Qian, M.Z. Papiz, P.J. Jackson, A.A. Brindley, I.W. Ng, J.D. Olsen, M.J. Dickman, P.A. Bullough, C.N. Hunter, Three-Dimensional Structure of the Rhodobacter sphaeroides RC- LH1-PufX Complex: Dimerization and Quinone Channels Promoted by PufX, Biochemistry, 52 (2013) 7575-7585. [DOI: 10.1021/bi4011946]

[24] S. Niwa, L.-J. Yu, K. Takeda, Y. Hirano, T. Kawakami, Z.-Y. Wang-Otomo, K. Miki, Structure of the LH1-RC complex from Thermochromatium tepidum at $3.0 \AA$, Nature, 508 (2014) 228-232. [DOI: 10.1038/nature13197]

[25] C.A. Siebert, P. Qian, D. Fotiadis, A. Engel, C.N. Hunter, P.A. Bullough, Molecular architecture of photosynthetic membranes in Rhodobacter sphaeroides: the role of PufX, EMBO J, 23 (2004) 690-700. [DOI: 10.1038/sj.emboj.7600092] 
[26] S. Scheuring, J.N. Sturgis, Atomic force microscopy of the bacterial photosynthetic apparatus: plain pictures of an elaborate machinery, Photosynth. Res., 102 (2009) 197-211. [DOI: 10.1007/s11120-009-9413-7]

[27] T.J. Aartsma, J. Matysik, Biophysical Techniques in Photosynthesis Volume II, Springer, Dordrecht, 2008.

[28] E. Hofmann, P.M. Wrench, F.P. Sharples, R.G. Hiller, W. Welte, K. Diederichs, Structural basis of light harvesting by carotenoids: peridinin-chlorophyll-protein from Amphidinium carterae, Science, 272 (1996) 1788-1791. [DOI: 10.1126/science.272.5269.1788]

[29] A.N. Macpherson, R.G. Hiller, Light-harvesting systems in chlorophyll c-containing algae, in: B.R. Green, W.W. Parson (Eds.) Light-harvesting antennas in photosynthesis, Kluwer Academic Publishers, Dordrecht, Boston, London, 2003, pp. 323-352.

[30] A. Beer, K. Gundermann, J. Beckmann, C. Büchel, Subunit composition and pigmentation of fucoxanthin-chlorophyll proteins in diatoms: evidence for a subunit involved in diadinoxanthin and diatoxanthin binding, Biochemistry, 45 (2006) 13046-13053. [DOI: 10.1021/bi061249h]

[31] R. Fujii, M. Kita, Y. Inuma, N. Oka, Y. Takaesu, T. Taira, M. Iha, R.J. Cogdell, H. Hashimoto, Isolation and purification of the major photosynthetic antenna, fucoxanthin-Chl a/c protein, from cultured discoid germilings of the brown Alga, Cladosiphon okamuranus TOKIDA (Okinawa Mozuku), Photosynth. Res., 111 (2012) 157-163. [DOI: 10.1007/s11120-011-9688-3]

[32] R. Fujii, M. Kita, M. Doe, Y. Iinuma, N. Oka, Y. Takaesu, T. Taira, M. Iha, T. Mizoguchi, R.J. Cogdell, H. Hashimoto, The pigment stoichiometry in a chlorophyll $a / c$ type photosynthetic antenna, Photosynth. Res., 111 (2012) 165-172. [DOI: 10.1007/s11120-011-9698-1]

[33] E. Papagiannakis, I.H.M. van Stokkum, H. Fey, C. Büchel, R. van Grondelle, Spectroscopic characterization of the excitation energy transfer in the fucoxanthin-chlorophyll protein of diatoms, Photosynth. Res., 86 (2005) 241-250. [DOI: $10.1007 / \mathrm{s} 11120-005-1003-8]$

[34] L. Premvardhan, D.J. Sandberg, H. Fey, R.R. Birge, C. Buchel, R. van Grondelle, The charge-transfer properties of the $\mathrm{S}_{2}$ state of fucoxanthin in solution and in fucoxanthin chlorophyll-a/c2 protein (FCP) based on stark spectroscopy and 
molecular-orbital theory, J. Phys. Chem. B, 112 (2008) 11838-11853. [DOI: $10.1021 / \mathrm{jp} 802689 \mathrm{p}]$

[35] L. Premvardhan, L. Bordes, A. Beer, C. Büchel, B. Robert, Carotenoid structures and environments in trimeric and oligomeric fucoxanthin chlorophyll $a / c_{2}$ proteins from resonance Raman spectroscopy, J. Phys. Chem. B, 113 (2009) 12565-12574. [10.1021/jp903029g]

[36] M. Di Valentin, E. Meneghin, L. Orian, A. Polimeno, C. Buchel, E. Salvadori, C.W. Kay, D. Carbonera, Triplet-triplet energy transfer in fucoxanthin-chlorophyll protein from diatom Cyclotella meneghiniana: insights into the structure of the complex, Biochim. Biophys. Acta., 1827 (2013) 1226-1234. [DOI: 10.1016/j.bbabio.2013.07.003] [37] A. Gelzinis, V. Butkus, E. Songaila, R. Augulis, A. Gall, C. Büchel, B. Robert, D. Abramavicius, D. Zigmantas, L. Valkunas, Mapping energy transfer channels in fucoxanthin-chlorophyll protein complex, Biochim. Biophys. Acta - Bioenergetics, 1847 (2015) 241-247. [DOI: 10.1016/j.bbabio.2014.11.004]

[38] D. Kosumi, M. Kita, R. Fujii, M. Sugisaki, N. Oka, Y. Takaesu, T. Taira, M. Iha, H. Hashimoto, Excitation Energy-Transfer Dynamics of Brown Algal Photosynthetic Antennas, J. Phys. Chem. Lett., 3 (2012) 2659-2664. [DOI: 10.1021/Jz300612c]

[39] A.W. Roszak, K. McKendrick, A.T. Gardiner, I.A. Mitchell, N.W. Isaacs, R.J. Cogdell, H. Hashimoto, H.A. Frank, Protein regulation of carotenoid binding: Gatekeeper and locking amino acid residues in reaction centers of Rhodobacter sphaeroides, Structure, 12 (2004) 765-773. [DOI: 10.1016/j.str.2004.02.037]

[40] N.J. Fraser, H. Hashimoto, R.J. Cogdell, Carotenoids and bacterial photosynthesis: The story so far ... Photosynthesis Research, 70 (2001) 249-256. [DOI: 10.1023/A:1014715114520]

[41] D. Xia, C.-A. Yu, H. Kim, J.-Z. Xia, A.M. Kachurin, L. Zhang, L. Yu, J. Deisenhofer, Crystal Structure of the Cytochrome $b c_{1}$ Complex from Bovine Heart Mitochondria, Science, 277 (1997) 60-66. [DOI: 10.1126/science.277.5322.60]

[42] M.M. Benning, G. Wesenberg, M.S. Caffrey, R.G. Bartsch, T.E. Meyer, M.A. Cusanovich, I. Rayment, H.M. Holden, Molecular Structure of Cytochrome $\mathrm{C}_{2}$ Isolated from Rhodobacter capsulatus Determined at 2.5 Å Resolution, J. Mol. Biol., 220 (1991) 673-685. [DOI: 10.1016/0022-2836(91)90109-J]

[43] D. Stock, A.G. Leslie, J.E. Walker, Molecular architecture of the rotary motor in ATP synthase, Science, 286 (1999) 1700-1705. [DOI: 10.1126/science.286.5445.1700] 
[44] S. Karrasch, P.A. Bullough, R. Ghosh, The $8.5 \AA$ projection map of the light-harvesting complex I from Rhodospirillum rubrum reveals a ring composed of 16 subunits, EMBO J., 14 (1995) 631-638.

[45] V. Sundström, T. Pullerits, R. van Grondelle, Photosynthetic Light-harvesting: Reconciling dynamics and structure of purple bacterial LH2 reveals function of photosynthetic unit, J. Phys. Chem. B, 103 (1999) 2327-2346. [DOI: 10.1021/jp983722+]

[46] H.A. Frank, Spectroscopic studies of the low-lying singlet excited electronic states and photochemical properties of carotenoids, Arch. Biochem. Biophys., 385 (2001) 53-60. [DOI: 10.1006/abbi.2000.2091]

[47] P. Tavan, K. Schulten, The low-lying electronic excitations in long polyenes: A PPP-MRD-CI study, J. Chem. Phys., 85 (1986) 6602-6609. [DOI: 10.1063/1.451442]

[48] P. Tavan, K. Schulten, Electronic excitations in finite and infinite polyenes, Phys. Rev. B, 36 (1987) 4337-4358. [DOI: 10.1103/PhysRevB.36.4337]

[49] T. Sashima, Y. Koyama, T. Yamada, H. Hashimoto, The $1 \mathrm{~B}_{\mathrm{u}^{+}}, 1 \mathrm{~B}_{\mathrm{u}^{-}}$, and $2 \mathrm{Ag}^{-}$ Energies of Crystalline Lycopene, $\beta$-Carotene, and Mini-9- $\beta$-carotene as Determined by Resonance-Raman Excitation Profiles: Dependence of the $1 \mathrm{Bu}^{-}$State Energy on the Conjugation Length, J. Phys. Chem. B, 104 (2000) 5011-5019. [DOI: $10.1021 / j p 994185 b]$

[50] A.N. Macpherson, J.B. Arellano, N.J. Fraser, R.J. Cogdell, T. Gillbro, Efficient Energy Transfer from the Carotenoid $\mathrm{S}_{2}$ State in a Photosynthetic Light-Harvesting Complex, Biophys. J., 80 (2001) 923-930. [DOI: 10.1016/s0006-3495(01)76071-7]

[51] F.S. Rondonuwu, K. Yokoyama, R. Fujii, Y. Koyama, R.J. Cogdell, Y. Watanabe, The role of the $1{ }^{1} \mathrm{~B}_{\mathrm{u}}{ }^{-}$state in carotenoid-to-bacteriochlorophyll singlet-energy transfer in the LH2 antenna complexes from Rhodobacter sphaeroides G1C, Rhodobacter sphaeroides 2.4.1, Rhodospirillum molischianum and Rhodopseudomonas acidophila, Chem. Phys. Lett., 390 (2004) 314-322. [DOI: 10.1016/j.cplett.2004.03.089]

[52] G. Cerullo, D. Polli, G. Lanzani, S. De Silvestri, H. Hashimoto, R.J. Cogdell, Photosynthetic light harvesting by carotenoids: detection of an intermediate excited state, Science, 298 (2002) 2395-2398. [DOI: 10.1126/science.1074685]

[53] D. Kosumi, M. Komukai, H. Hashimoto, M. Yoshizawa, Ultrafast Dynamics of All-trans- $\beta$-Carotene Explored by Resonant and Nonresonant Photoexcitations, Phys. Rev. Lett., 95 (2005) 213601-213604. [DOI: 10.1103/PhysRevLett.95.213601] 
[54] H. Hashimoto, K. Yanagi, M. Yoshizawa, D. Polli, G. Cerullo, G. Lanzani, S. De Silvestri, A.T. Gardiner, R.J. Cogdell, The very early events following photoexcitation of carotenoids, Arch. Biochem. Biophys., 430 (2004) 61-69. [DOI: 10.1016/j.abb.2004.04.022]

[55] E.E. Ostroumov, R.M. Mulvaney, R.J. Cogdell, G.D. Scholes, Broadband 2D Electronic Spectroscopy Reveals a Carotenoid Dark State in Purple Bacteria, Science, 340 (2013) 52-56. [DOI: 10.1126/science.1230106]

[56] C.C. Gradinaru, J.T. Kennis, E. Papagiannakis, I.H. van Stokkum, R.J. Cogdell, G.R. Fleming, R.A. Niederman, R. van Grondelle, An unusual pathway of excitation energy deactivation in carotenoids: singlet-to-triplet conversion on an ultrafast timescale in a photosynthetic antenna, Proc. Natl. Acad. Sci. USA, 98 (2001) 2364-2369. [DOI: 10.1073/pnas.051501298]

[57] E. Papagiannakis, S.K. Das, A. Gall, I.H.M. van Stokkum, B. Robert, R. van Grondelle, H.A. Frank, J.T.M. Kennis, Light harvesting by carotenoids incorporated into the B850 light-harvesting complex from Rhodobacter sphaeroides R-26.1: Excited-state relaxation, ultrafast triplet formation, and energy transfer to bacteriochlorophyll, J. Phys. Chem. B, 107 (2003) 5642-5649. [DOI: 10.1021/jp027174i]

[58] E. Papagiannakis, I.H.M. van Stokkum, R. van Grondelle, R.A. Niederman, D. Zigmantas, V. Sundström, T. Polívka, A near-infrared transient absorption study of the excited-state dynamics of the carotenoid spirilloxanthin in solution and in the LH1 complex of Rhodospirillum rubrum, J. Phys. Chem. B, 107 (2003) 11216-11223. [DOI: $10.1021 / \mathrm{jp} 034931 \mathrm{j}]$

[59] W. Wohlleben, T. Buckup, H. Hashimoto, R.J. Cogdell, J.L. Herek, M. Motzkus, Pump-deplete-probe spectroscopy and the puzzle of carotenoid dark states, J. Phys. Chem. B, 108 (2004) 3320-3325. [DOI: 10.1021/jp036145k]

[60] D. Kosumi, S. Maruta, T. Horibe, Y. Nagaoka, R. Fujii, M. Sugisaki, R.J. Cogdell, H. Hashimoto, Ultrafast excited state dynamics of spirilloxanthin in solution and bound to core antenna complexes: identification of the $\mathrm{S}^{*}$ and $\mathrm{T}_{1}$ states, J. Chem. Phys., 137 (2012) 064505. [DOI: 10.1063/1.4737129]

[61] P.O. Andersson, T. Gillbro, Photophysics and Dynamics of the Lowest Excited Singlet-State in Long Substituted Polyenes with Implications to the Very Long-Chain Limit, J. Chem. Phys., 103 (1995) 2509-2519. [DOI: 10.1063/1.469672] 
[62] T. Polívka, D. Zigmantas, H.A. Frank, J.A. Bautista, J.L. Herek, Y. Koyama, R. Fujii, V. Sundström, Near-Infrared Time-Resolved Study of the $\mathrm{S}_{1}$ State Dynamics of the Carotenoid Spheroidene, J. Phys. Chem. B, 105 (2001) 1072-1080. [DOI: $10.1021 /$ jp002206s]

[63] H. Hörvin Billsten, D. Zigmantas, V. Sundström, T. Polívka, Dynamics of vibrational relaxation in the $\mathrm{S}_{1}$ state of carotenoids having 11 conjugated $\mathrm{C}=\mathrm{C}$ bonds, Chem. Phys. Lett., 355 (2002) 465-470. [DOI: 10.1016/s0009-2614(02)00268-3]

[64] J.A. Bautista, R.E. Connors, B.B. Raju, R.G. Hiller, F.P. Sharples, D. Gosztola, M.R. Wasielewski, H.A. Frank, Excited state properties of peridinin: Observation of a solvent dependence of the lowest excited singlet state lifetime and spectral behavior unique among carotenoids, J. Phys. Chem. B, 103 (1999) 8751-8758. [DOI: $10.1021 /$ jp9916135]

[65] H.A. Frank, J.A. Bautista, J. Josue, Z. Pendon, R.G. Hiller, F.P. Sharples, D. Gosztola, M.R. Wasielewski, Effect of the solvent environment on the spectroscopic properties and dynamics of the lowest excited states of carotenoids, J. Phys. Chem. B, 104 (2000) 4569-4577. [DOI: 10.1021/jp000079u]

[66] M. Yoshizawa, H. Aoki, H. Hashimoto, Vibrational relaxation of the $2 \mathrm{Ag}^{-}$excited state in all-trans- $\beta$-carotene obtained by femtosecond time-resolved Raman spectroscopy, Phys. Rev. B, $63 \quad$ (2001) 180301(R). [DOI:
[DO 10.1103/PhysRevB.63.180301]

[67] M. Yoshizawa, H. Aoki, H. Hashimoto, Femtosecond Time-Resolved Raman Signals on Ultrafast Dynamics in All-trans- $\beta$-Carotene, Bull. Chem. Soc. Jpn., 75 (2002) 949-955. [DOI: 10.1246/bcsj.75.949]

[68] D.W. McCamant, J.E. Kim, R.A. Mathies, Vibrational Relaxation in beta-Carotene Probed by Picosecond Stokes and Anti-Stokes Resonance Raman Spectroscopy, J. Phys. Chem. A, 106 (2002) 6030-6038. [DOI: 10.1021/jp0203595]

[69] D. Zigmantas, T. Polivka, R.G. Hiller, A. Yartsev, V. Sundstrom, Spectroscopic and dynamic properties of the peridinin lowest singlet excited states, J. Phys. Chem. A, 105 (2001) 10296-10306. [DOI :10.1021/jp010022n]

[70] D. Zigmantas, R.G. Hiller, V. Sundström, T. Polívka, Carotenoid to chlorophyll energy transfer in the peridinin-chlorophyll-a-protein complex involves an intramolecular charge transfer state, Proc. Natl. Acad. Sci. USA, 99 (2002) 16760-16765. [DOI: 10.1073/pnas.262537599] 
[71] D. Kosumi, T. Kusumoto, R. Fujii, M. Sugisaki, Y. Inuma, N. Oka, Y. Takaesu, T. Taira, M. Iha, H.A. Frank, H. Hashimoto, One- and two-photon pump-probe optical spectroscopic measurements reveal the $\mathrm{S}_{1}$ and intramolecular charge transfer states are distinct in fucoxanthin, Chem. Phys. Lett., 483 (2009) 95-100. [DOI: 10.1016/j.cplett.2009.10.077]

[72] D. Kosumi, T. Kusumoto, R. Fujii, M. Sugisaki, Y. Iinuma, N. Oka, Y. Takaesu, T. Taira, M. Iha, H.A. Frank, H. Hashimoto, Ultrafast excited state dynamics of fucoxanthin: excitation energy dependent intramolecular charge transfer dynamics, Phys. Chem. Chem. Phys., 13 (2011) 10762-10770. [DOI: 10.1039/c0cp02568b]

[73] D. Kosumi, T. Kusumoto, R. Fujii, M. Sugisaki, Y. Inuma, N. Oka, Y. Takaesu, T. Taira, M. Iha, H.A. Frank, H. Hashimoto, Ultrafast $\mathrm{S}_{1}$ and ICT state dynamics of a marine carotenoid probed by femtosecond one- and two-photon pump-probe spectroscopy, J. Lumin., 131 (2011) 515-518. [DOI:10.1016/j.jlumin.2010.09.018]

[74] D. Kosumi, R. Fujii, M. Sugisaki, N. Oka, M. Iha, H. Hashimoto, Characterization of the intramolecular transfer state of marine carotenoid fucoxanthin by femtosecond pump-probe spectroscopy, Photosynth. Res., 121 (2014) 61-68. [DOI: 10.1007/s11120-014-9995-6]

[75] D. Kosumi, T. Kajikawa, S. Okumura, M. Sugisaki, K. Sakaguchi, S. Katsumura, H. Hashimoto, Elucidation and Control of an Intramolecular Charge Transfer Property of Fucoxanthin by a Modification of Its Polyene Chain Length, J. Phys. Chem. Lett., 5 (2014) 792-797. [DOI: 10.1021/jz5000287]

[76] T. Polívka, V. Sundström, Ultrafast dynamics of carotenoids excited states - From solution to natural and artificial systems., Chem. Rev., 104 (2004) 2021-2071. [DOI: $10.1021 / \mathrm{cr} 020674 \mathrm{n}]$

[77] D. Kosumi, T. Kajikawa, K. Yano, S. Okumura, M. Sugisaki, K. Sakaguchi, S. Katsumura, H. Hashimoto, Roles of allene-group in an intramolecular charge transfer character of a short fucoxanthin homolog as revealed by femtosecond pump-probe spectroscopy, Chem. Phys. Lett., 602 (2014) 75-79. [DOI: 10.1016/j.cplett.2014.04.022] [78] H. Hashimoto, M. Sugisaki, M. Yoshizawa, Ultrafast time-resolved vibrational spectroscopies of carotenoids in photosynthesis, Biochim. Biophys. Acta Bioenergetics, 1847 (2015) 69-78. [DOI: 10.1016/j.bbabio.2014.09.001]

[79] K. Onaka, R. Fujii, H. Nagae, M. Kuki, Y. Koyama, Y. Watanabe, The state energy 
and the displacements of the potential minima of the $2 \mathrm{Ag}^{-}$state in all-trans- $\beta$-carotene as determined by fluorescence spectroscopy, Chem. Phys. Lett., 315 (1999) 75-81. [DOI: 10.1016/s0009-2614(99)01212-9]

[80] R.A. Auerbach, R.L. Christensen, M.F. Granville, B.E. Kohler, Absorption and Emission of 2,12-Dimethyltridecahexaene, J. Chem. Phys., 74 (1981) 4-9. [DOI: $10.1063 / 1.440857]$

[81] H. Hashimoto, Y. Koyama, The $\mathrm{C}=\mathrm{C}$ stretching Raman lines of $\beta$-carotene isomers in the $\mathrm{S}_{1}$ state as detected by pump-probe resonance Raman spectroscopy, Chem. Phys. Lett., 154 (1989) 321-325. [DOI: 10.1016/0009-2614(89)85363-1]

[82] H. Hashimoto, Y. Koyama, Raman spectra of all-trans- $\beta$-carotene in the $\mathrm{S}_{1}$ and $\mathrm{T}_{1}$ states produced by direct photoexcitation, Chem. Phys. Lett., 163 (1989) 251-256. [DOI: 10.1016/0009-2614(89)80045-4]

[83] M. Yoshizawa, M. Kurosawa, Femtosecond time-resolved Raman spectroscopy using stimulated Raman scattering, Phys. Rev. A, 61 (1999) 013808. [DOI: 10.1103/PhysRevA.61.013808]

[84] P. Kukura, D.W. McCamant, R.A. Mathies, Femtosecond stimulated Raman spectroscopy, Annu. Rev. Phys. Chem., 58 (2007) 461-488. [DOI: 10.1146/annurev.physchem.58.032806.104456]

[85] R.R. Frontiera, R.A. Mathies, Femtosecond stimulated Raman spectroscopy, Laser Photonics Rev., 5 (2011) 102-113. [DOI: 10.1002/lpor.200900048]

[86] J.R. Challa, Y. Du, D.W. McCamant, Femtosecond Stimulated Raman Spectroscopy Using a Scanning Multichannel Technique, Appl. Spectrosc., 66 (2012) 227-232. [DOI: 10.1366/11-06457]

[87] M. Yoshizawa, H. Aoki, M. Ue, H. Hashimoto, Ultrafast relaxation kinetics of excited states in a series of mini- and macro- $\beta$-carotenes, Phys. Rev. B, 67 (2003) 174302. [DOI: 10.1103/PhysRevB.67.174302]

[88] D. Kosumi, K. Yanagi, T. Nishio, H. Hashimoto, M. Yoshizawa, Excitation energy dependence of excited states dynamics in all-trans-carotenes determined by femtosecond absorption and fluorescence spectroscopy, Chem. Phys. Lett., 408 (2005) 89-95. [DOI: 10.1016/j.cplett.2005.04.006]

[89] M. Sugisaki, K. Yanagi, R.J. Cogdell, H. Hashimoto, Unified explanation for linear and nonlinear optical responses in $\beta$-carotene: A sub-20 fs degenerate four-wave mixing spectroscopic study, Phys. Rev. B, 75 (2007) 155110. [DOI:
[DI) 
10.1103/PhysRevB.75.155110]

[90] P.J. Walla, P.A. Linden, K. Ohta, G.R. Fleming, Excited-State Kinetics of the Carotenoid $\mathrm{S}_{1}$ State in LHC II and Two-Photon Excitation Spectra of Lutein and $\beta$ -Carotene in Solution: Efficient Car $\mathrm{S}_{1} \rightarrow$ Chl Electronic Energy Transfer via Hot $\mathrm{S}_{1}$ States?, J. Phys. Chem. A, 106 (2002) 1909-1916. [DOI: 10.1021/jp011495x]

[91] D. Kosumi, K. Abe, H. Karasawa, M. Fujiwara, R.J. Cogdell, H. Hashimoto, M. Yoshizawa, Ultrafast relaxation kinetics of the dark $\mathrm{S}_{1}$ state in all-trans- $\beta$-carotene explored by one- and two-photon pump-probe spectroscopy, Chem. Phys., 373 (2010) 33-37. [DOI: 10.1016/j.chemphys.2009.12.013]

[92] C.K.N. Patel, R.E. Slusher, Photon Echoes in Gases, Phys. Rev. Lett., 20 (1968) 1087-1089. [DOI: 10.1103/PhysRevLett.20.1087]

[93] I. Pastirk, V.V. Lozovoy, M. Dantus, Femtosecond photon echo and virtual echo measurements of the vibronic and vibrational coherence relaxation times of iodine vapor, Chem. Phys. Lett., 333 (2001) 76-82. [DOI: 10.1016/S0009-2614(00)01334-8]

[94] B.I. Grimberg, V.V. Lozovoy, M. Dantus, S. Mukamel, Ultrafast Nonlinear spectroscopic techniques in the gas phase and their density matrix representation, $\mathrm{J}$. Phys. Chem. A, 106 (2002) 697-718. [DOI: 10.1021/jp0104511]

[95] A.H. Zewail, Femtochemistry: Atomic-Scale Dynamics of the Chemical Bond, J. Phys. Chem. A, 104 (2000) 5660-5694. [DOI: 10.1021/jp001460h]

[96] S. Mukamel, Principles of Nonlinear Optical Spectroscopy, Oxford University Press, New York, Oxford, 1995.

[97] R.W. Boyd, Nonlinear Optics 3rd ed., Academic Press, Amsterdam, 2008.

[98] T. Siebert, V. Engel, A. Materny, W. Kiefer, M. Schmitt, Probing the kinetics of a nonadiabatic transition initiating out of vibrationally excited as well as ground state modes with femtosecond time-resolved transient gratings, J. Phys. Chem. A, 107 (2003) 8355-8362. [DOI: 10.1021/jp022650q]

[99] T. Hornung, H. Skenderović, M. Motzkus, Observation of all-trans- $\beta$-carotene wavepacket motion on the electronic ground and excited dark state using degenerate four-wave mixing (DFWM) and pump-DFWM, Chem. Phys. Lett., 402 (2005) 283-288. [DOI: 10.1016/j.cplett.2004.11.135]

[100] J. Hauer, T. Buckup, M. Motzkus, Pump-degenerate four wave mixing as a technique for analyzing structural and electronic evolution: multidimensional time-resolved dynamics near a conical intersection, J. Phys. Chem. A, 111 (2007) 
10517-10529. [DOI: 10.1021/jp073727j]

[101] M. Sugisaki, M. Fujiwara, K. Yanagi, R.J. Cogdell, H. Hashimoto, Four-wave mixing signals from $\beta$-carotene and its $n=15$ homologue, Photosyn. Res., 95 (2008) 299-308. [DOI: 10.1007/s11120-007-9265-y]

[102] T. Buckup, J. Hauer, J. Mohring, M. Motzkus, Multidimensional spectroscopy of $\beta$-carotene: vibrational cooling in the excited state, Arch. Biochem. Biophys., 483 (2009) 219-223. [DOI: 10.1016/j.abb.2008.10.031]

[103] M. Fujiwara, K. Yamauchi, M. Sugisaki, A. Gall, B. Robert, R.J. Cogdell, H. Hashimoto, Energy dissipation in the ground-state vibrational manifolds of $\beta$-carotene homologues: A sub-20-fs time-resolved transient grating spectroscopic study, Phys. Rev. B, 77 (2008) 205118. [DOI: 10.1103/PhysRevB.77.205118]

[104] M. Sugisaki, M. Fujiwara, S.V. Nair, H.E. Ruda, R.J. Cogdell, H. Hashimoto, Excitation-energy dependence of transient grating spectroscopy in $\beta$-carotene, Phys. Rev. B, 80 (2009) 035118. [DOI: 10.1103/PhysRevB.80.035118]

[105] N. Christensson, T. Polivka, A. Yartsev, T. Pullerits, Photon echo spectroscopy reveals structure-dynamics relationships in carotenoids, Phys. Rev. B, 79 (2009) 245118. [DOI: 10.1103/PhysRevB.79.245118]

[106] M. Fujiwara, M. Sugisaki, A. Gall, B. Robert, R.J. Cogdell, H. Hashimoto, Ultrafast optical responses of $\beta$-carotene and lycopene probed by sub-20-fs time-resolved coherent spectroscopy, J. Lumin., 129 (2009) 1808-1812. [DOI: 10.1016/j.jlumin.2009.04.098]

[107] M. Sugisaki, M. Fujiwara, D. Kosumi, R. Fujii, M. Nango, R.J. Cogdell, H. Hashimoto, Comparison of transient grating signals from spheroidene in an organic solvent and in pigment-protein complexes from Rhodobacter sphaeroides 2.4.1, Phys. Rev. B, 81 (2010) 245112. [DOI: 10.1103/PhysRevB.81.245112]

[108] M. Sugisaki, D. Kosumi, K. Saito, R.J. Cogdell, H. Hashimoto, Control of coherent vibronic oscillations in $\beta$-carotene by ultrashort laser pulses, Phys. Status Solidi C, 8 (2011) 151-154. [DOI: 10.1002/pssc.201000679]

[109] M. Sugisaki, D. Kosumi, K. Saito, R.J. Cogdell, H. Hashimoto, Strong coherent coupling of vibronic oscillations in spheroidene, Physics Procedia, 13 (2011) 74-77. [DOI:10.1016/j.phpro.2011.02.018]

[110] M. Sugisaki, M. Fujiwara, R. Fujii, K. Nakagawa, M. Nango, R.J. Cogdell, H. Hashimoto, Transient grating spectroscopy in photosynthetic purple bacteria 
Rhodobacter sphaeroides 2.4.1, J. Lumin., 129 (2009) 1908-1911. [DOI: 10.1016/j.jlumin.2009.01.027]

[111] M.H. Vos, J.C. Lambry, S.J. Robles, D.C. Youvan, J. Breton, J.L. Martin, Direct Observation of Vibrational Coherence in Bacterial Reaction Centers Using Femtosecond Absorption-Spectroscopy, Proc. Nat. Acad. Sci. U.S.A, 88 (1991) 8885-8889. [DOI: 10.1073/pnas.88.20.8885]

[112] M.H. Vos, F. Rappaport, J.C. Lambry, J. Breton, J.L. Martin, Visualization of Coherent Nuclear Motion in a Membrane-Protein by Femtosecond Spectroscopy, Nature, 363 (1993) 320-325. [DOI 10.1038/363320a0]

[113] M.H. Vos, M.R. Jones, C.N. Hunter, J. Breton, J.-L. Martin, Coherent nuclear dynamics at room temperature in bacterial reactioncenters, Proc. Natl. Acad. Sci. USA, 91 (1994) 12701-12705.

[114] R. Jimenez, F. vanMourik, J.Y. Yu, G.R. Fleming, Three-pulse photon echo measurements on LH1 and LH2 complexes of Rhodobacter sphaeroides: A nonlinear spectroscopic probe of energy transfer, Journal of Physical Chemistry B, 101 (1997) 7350-7359. [DOI: 10.1021/jp970299g]

[115] M.-L. Groot, J.-Y. Yu, R. Agarwal, J.R. Norris, G.R. Fleming, Three-Pulse Photon Echo Measurements on the Accessory Pigments in the Reaction Center of Rhodobacter sphaeroides, J. Phys. Chem. B, 102 (1998) 5923-5931. [DOI: 10.1021/jp9808680]

[116] R. Agarwal, M. Yang, Q.H. Xu, G.R. Fleming, Three pulse photon echo peak shift study of the B800 band of the LH2 complex of Rps. acidophila at room temperature: A coupled master equation and nonlinear optical response function approach, J. Phys. Chem. B, 105 (2001) 1887-1894. [DOI: 10.1021/jp0031146]

[117] K.R. Shelly, E.A. Carson, W.F. Beck, Vibrational coherence from the dipyridine complex of bacteriochlorophyll $a$ : intramolecular modes in the $10-220-\mathrm{cm}^{-1}$ regime, intermolecular solvent modes, and relevance to photosynthesis, J. Am. Chem. Soc., 125 (2003) 11810-11811. [DOI: 10.1021/ja0366890]

[118] K.R. Shelly, E.C. Golovich, W.F. Beck, Intermolecular vibrational coherence in bacteriochlorophyll a with clustered polar solvent molecules, J. Phys. Chem. B, 110 (2006) 20586-20595. [DOI: 10.1021/jp062909v]

[119] G.S. Engel, T.R. Calhoun, E.L. Read, T.K. Ahn, T. Mancal, Y.C. Cheng, R.E. Blankenship, G.R. Fleming, Evidence for wavelike energy transfer through quantum coherence in photosynthetic systems, Nature, 446 (2007) 782-786. [DOI: 
10.1038 /nature05678]

[120] H. Lee, Y.C. Cheng, G.R. Fleming, Coherence dynamics in photosynthesis: protein protection of excitonic coherence, Science, 316 (2007) 1462-1465. [DOI: 10.1126/science.1142188]

[121] E. Collini, G.D. Scholes, Coherent Intrachain Energy Migration in a Conjugated Polymer at Room Temperature, Science, 323 (2009) 369-373. [DOI: 10.1126/science.1164016]

[122] K. Ishii, H. Hamaguchi, Picosecond time-resolved multiplex CARS spectroscopy using optical Kerr gating, Chem. Phys. Lett., 367 (2003) 672-677. [DOI: 10.1016/S0009-2614(02)01740-2]

[123] T. Siebert, M. Schmitt, V. Engel, A. Materny, W. Kiefer, Population dynamics in vibrational modes during non-born-Oppenheimer Processes: CARS spectroscopy used as a mode-selective filter, J. Am. Chem. Soc., 124 (2002) 6242-6243. [DOI: $10.1021 / \mathrm{ja} 0173831]$

[124] T. Siebert, R. Maksimenka, A. Materny, V. Engel, W. Kiefer, M. Schmitt, The role of specific normal modes during non-Born-Oppenheimer dynamics: the S1-S0 internal conversion of $\beta$-carotene interrogated on a femtosecond time-scale with coherent anti-Stokes Raman scattering, J. Raman Spectrosc., 33 (2002) 844-854. [DOI: 10.1002/jrs.926]

[125] T. Siebert, M. Schmitt, S. Grafe, V. Engel, Ground state vibrational wave-packet and recovery dynamics studied by time-resolved CARS and pump-CARS spectroscopy, J. Raman Spectrosc., 37 (2006) 397-403. [DOI: 10.1002/jrs.1441]

[126] G. Cerullo, G. Lanzani, M. Zavelani-Rossi, S. De Silvestri, Early events of energy relaxation in all-trans- $\beta$-carotene following sub-10 fs optical-pulse excitation, Phys. Rev. B, 63 (2001) 241104(R). [DOI: 10.1103/PhysRevB.63.241104]

[127] G. Lanzani, G. Cerullo, M. Zavelani-Rossi, S. De Silvestri, Sub-10 fs time resolved study of excited state relaxation in all-trans- $\beta$-carotene, Synth. Metals, 116 (2001) 1-3. [DOI: 10.1016/s0379-6779(00)00502-6]

[128] D. Polli, G. Cerullo, G. Lanzani, S. De Silvestri, H. Hashimoto, R.J. Cogdell, Carotenoid-bacteriochlorophyll energy transfer in LH2 complexes studied with 10-fs time resolution, Biophys. J., 90 (2006) 2486-2497. [DOI: 10.1529/biophysj.105.069286]

[129] D. Polli, M.R. Antognazza, D. Brida, G. Lanzani, G. Cerullo, S. De Silvestri, 
Broadband pump-probe spectroscopy with sub-10-fs resolution for probing ultrafast internal conversion and coherent phonons in carotenoids, Chem. Phys., 350 (2008) 45-55. [DOI: 10.1016/j.chemphys.2007.12.021]

[130] J. Hauer, H. Skenderovic, K.-L. Kompa, M. Motzkus, Enhancement of Raman modes by coherent control in $\beta$-carotene, Chem. Phys. Lett., 421 (2006) 523-528. [DOI: 10.1016/j.cplett.2006.01.115]

[131] J. Konradi, A.K. Singh, A.V. Scaria, A. Materny, Selective spectral filtering of molecular modes of $\beta$-carotene in solution using optimal control in four-wave-mixing spectroscopy, J. Raman Spectrosc., 37 (2006) 697-704. [DOI: 10.1002/jrs.1502]

[132] J.L. Herek, W. Wohlleben, R.J. Cogdell, D. Zeidler, M. Motzkus, Quantum control of energy flow in light harvesting, Nature, 417 (2002) 533-535. [DOI: $10.1038 / 417533 \mathrm{a}]$

[133] W. Wohlleben, T. Buckup, J.L. Herek, M. Motzkus, Coherent control for spectroscopy and manipulation of biological dynamics, ChemPhysChem, 6 (2005) 850-857. [DOI: 10.1002/cphc.200400414]

[134] J. Savolainen, R. Fanciulli, N. Dijkhuizen, A.L. Moore, J. Hauer, T. Buckup, M. Motzkus, J.L. Herek, Controlling the efficiency of an artificial light-harvesting complex, Proc. Natl. Acad. Sci. USA, 105 (2008) 7641-7646. [DOI: 10.1073/pnas.0711927105]

[135] H.A. Frank, Incorporation of carotenoids into reaction center and light-harvesting pigment-protein complexes, in: H.A. Frank, A.J. Young, G. Britton, R.J. Cogdell (Eds.) The Photochemistry of Carotenoids, Kluwer Academic Publishers, Dordrecht, 1999, pp. 235-244.

[136] J.F. Miller, S.B. Hinchigeri, P.S. Parkes-Loach, P.M. Callahan, J.R. Sprinkle, J.R. Riccobono, P.A. Loach, Isolation and characterization of a subunit form of the light-harvesting complex of Rhodospirillum rubrum, Biochemistry, 26 (1987) 5055-5062. [DOI: 10.1021/bi00390a026]

[137] P.S. Parkes-Loach, J.R. Sprinkle, P.A. Loach, Reconstitution of the B873 light-harvesting complex of Rhodospirillum rubrum from the separately isolated $\alpha$ - and $\beta$-polypeptides and bacteriochlorophyll $a$, Biochemistry, 27 (1988) 2718-2727. [DOI: 10.1021/bi00408a011]

[138] B.A. Heller, P.A. Loach, Isolation and Characterization of a Subunit Form of the B875 Light-Harvesting Complex from Rhodobacter capsulatus, Photochemistry and Photobiology, 51 (1990) 621-627. [DOI: 10.1111/j.1751-1097.1990.tb01975.x] 
[139] M.C. Chang, L. Meyer, P.A. Loach, Isolation and Characterization of a Structural Subunit from the Core Light-Harvesting Complex of Rhodobacter sphaeroides 2.4.1 and puc705-BA, Photochem. Photobiol., 52 (1990) 873-881. [DOI: 10.1111/j.1751-1097.1990.tb08696.x]

[140] P.L. Bustamante, Reconstitution of the Bacterial Core Light-harvesting Complexes of Rhodobacter sphaeroides and Rhodospirillum rubrum with Isolated $\alpha$ and $\beta$-Polypeptides, Bacteriochlorophyll $a$, and Carotenoid, J. Biol. Chem., 270 (1995) 5793-5804. [DOI: 10.1074/jbc.270.11.5793]

[141] J.B. Todd, P.S. Parkes-Loach, J.F. Leykam, P.A. Loach, In vitro reconstitution of the core and peripheral light-harvesting complexes of Rhodospirillum molischianum from separately isolated components, Biochemistry, 37 (1998) 17458-17468. [DOI: 10.1021/bi981114e]

[142] J. Akahane, F.S. Rondonuwu, L. Fiedor, Y. Watanabe, Y. Koyama, Dependence of singlet-energy transfer on the conjugation length of carotenoids reconstituted into the LH1 complex from Rhodospirillum rubrum G9, Chem. Phys. Lett., 393 (2004) 184-191. [DOI: 10.1016/j.cplett.2004.06.021]

[143] L. Fiedor, J. Akahane, Y. Koyama, Carotenoid-induced cooperative formation of bacterial photosynthetic LH1 complex, Biochemistry, 43 (2004) 16487-16496. [DOI: 10.1021/bi0481287]

[144] Y. Kakitani, J. Akahane, H. Ishii, H. Sogabe, H. Nagae, Y. Koyama, Conjugation-length dependence of the $\mathrm{T} 1$ lifetimes of carotenoids free in solution and incorporated into the LH2, LH1, RC, and RC-LH1 complexes: possible mechanisms of triplet-energy dissipation, Biochemistry, 46 (2007) 2181-2197. [DOI: $10.1021 / \mathrm{bi062237z]}$

[145] K. Nakagawa, S. Suzuki, R. Fujii, A.T. Gardiner, R.J. Cogdell, M. Nango, H. Hashimoto, Probing the effect of the binding site on the electrostatic behavior of a series of carotenoids reconstituted into the light-harvesting 1 complex from purple photosynthetic bacterium Rhodospirillum rubrum detected by Stark spectroscopy, J. Phys. Chem. B, 112 (2008) 9467-9475. [DOI: 10.1021/jp801773j]

[146] K. Nakagawa, S. Suzuki, R. Fujii, A.T. Gardiner, R.J. Cogdell, M. Nango, H. Hashimoto, Probing binding site of bacteriochlorophyll $a$ and carotenoid in the reconstituted LH1 complex from Rhodospirillum rubrum S1 by Stark spectroscopy, Photosynth. Res., 95 (2008) 339-344. [DOI: 10.1007/s11120-007-9261-2] 
[147] T. Horibe, K. Nakagawa, T. Kusumoto, R. Fujii, R.J. Cogdell, M. Nango, H. Hashimoto, Polarization angle dependence of stark absorption spectra of spirilloxanthin bound to the reconstituted LH1 complexes using LH1-subunits isolated from the purple photosynthetic bacterium Rhodospirillum rubrum, Acta Biochim. Polon., 59 (2012) 97-100.

[148] M. Yamamoto, T. Horibe, Y. Nishisaka, S. Suzuki, M. Kozaki, R. Fujii, M. Doe, M. Nango, K. Okada, H. Hashimoto, Reassociation of All-trans-3,4-Dihydroanhydrorhodovibrin with LH1 Subunits Isolated from Rhodospirillum rubrum: Selective Binding of All-trans Isomer from Mixture of cis- and trans-Isomers, Bulletin of the Chemical Society of Japan, 86 (2013) 121-128. [DOI: 10.1246/bcsj.20120230]

[149] Y. Koyama, Structures and functions of carotenoids in photosynthetic systems, J. Photochem. Photobiol. B: Biology, 9 (1991) 265-280. [DOI:
[DO 10.1016/1011-1344(91)80165-e]

[150] S. Kita, R. Fujii, R.J. Cogdell, H. Hashimoto, Characterization of fucoxanthin aggregates in mesopores of silica gel: Electronic absorption and circular dichroism spectroscopies, J. Photochem. Photobiol. A: Chemistry, (2015) in press. [DOI: 10.1016/j.jphotochem.2015.05.009]

[151] M. Kasha, Energy Transfer Mechanisms and the Molecular Exciton Model for Molecular Aggregates, Radiation Res., 20 (1963) 55-71. [DOI: 10.2307/3571331]

[152] M. Kasha, H.R. Rawls, M. Ashraf El-Bayoumi, The exciton model in molecular spectroscopy, Pure Appl. Chem., 11 (1965) 371-392. [DOI: 10.1351/pac196511030371]

[153] C. Hettich, C. Schmitt, J. Zitzmann, S. Kuhn, I. Gerhardt, V. Sandoghdar, Nanometer resolution and coherent optical dipole coupling of two individual molecules, Science, 298 (2002) 385-389. [DOI: 10.1126/science.1075606]

[154] F. Zsila, Z. Bikadi, J. Deli, M. Simonyi, Chiral detection of carotenoid assemblies, Chirality, 13 (2001) 446-453. [DOI: 10.1002/chir.1060]

[155] F. Zsila, Z. Bikádi, Z. Keresztes, J. Deli, M. Simonyi, Investigation of the Self-Organization of Lutein and Lutein Diacetate by Electronic Absorption, Circular Dichroism Spectroscopy, and Atomic Force Microscopy, J. Phys. Chem. B, 105 (2001) 9413-9421. [DOI: 10.1021/jp011626u]

[156] M. Simonyi, Z. Bikadi, F. Zsila, J. Deli, Supramolecular exciton chirality of carotenoid aggregates, Chirality, 15 (2003) 680-698. [DOI: 10.1002/chir.10282] 
[157] H.H. Billsten, V. Sundström, T. Polívka, Self-assembled aggregates of the carotenoid zeaxanthin: Time-resolved study of excited states, J. Phys. Chem. A, 109 (2005) 1521-1529. [DOI: 10.1021/jp044847j]

[158] F.C. Spano, Analysis of the UV/Vis and CD spectral line shapes of carotenoid assemblies: spectral signatures of chiral H-aggregates, J. Am. Chem. Soc., 131 (2009) 4267-4278. [DOI: 10.1021/ja806853v]

[159] C. Wang, M.J. Tauber, High-yield singlet fission in a zeaxanthin aggregate observed by picosecond resonance Raman spectroscopy, J. Am. Chem. Soc., 132 (2010) 13988-13991. [DOI: 10.1021/ja102851m]

[160] C. Wang, C.J. Berg, C.C. Hsu, B.A. Merrill, M.J. Tauber, Characterization of carotenoid aggregates by steady-state optical spectroscopy, J. Phys. Chem. B, 116 (2012) 10617-10630. [DOI: 10.1021/jp3069514]

[161] T. Ohnishi, M. Hatakeyama, N. Yamamoto, H. Tsubomura, Electrical and Spectroscopic Investigations of Molecular Layers of Fatty Acids Including Carotene, Bull. Chem. Soc. Jpn., 51 (1978) 1714-1716. [DOI: 10.1246/bcsj.51.1714]

[162] H. Hashimoto, D. Kiyohara, Y. Kamo, H. Komuta, Y. Mori, Molecular Orientation of all-trans- $\beta$-Carotene in Spin-Coated Film and in Langmuir-Blodgett Film as Detected by Polarized Optical Absorption and Reflection Spectroscopies, Jpn. J. Appl. Phys., 35 (1996) 281-289. [DOI: 10.1143/JJAP.35.281]

[163] K. Sakakibara, S. Ifuku, Y. Tsujii, H. Kamitakahara, T. Takano, F. Nakatsubo, Langmuir-Blodgett films of a novel cellulose derivative with dihydrophytyl group: the ability to anchor beta-carotene molecules, Biomacromolecules, 7 (2006) 1960-1967. [DOI: $10.1021 / \mathrm{bm} 060083 \mathrm{x}$ ]

[164] H. Auweter, H. Haberkorn, W. Heckmann, D. Horn, E. Lüddecke, J. Rieger, H. Weiss, Supramolecular Structure of Precipitated Nanosize $\beta$-Carotene Particles, Angew. Chem. Int. Ed., 38 (1999) 2188-2191. [DOI: 10.1002/(sici)1521-3773(19990802)38:15<2188::aid-anie2188>3.0.co;2-\#]

[165] E. Karabudak, W. Wohlleben, H. Colfen, Investigation of $\beta$-carotene-gelatin composite particles with a multiwavelength $\mathrm{UV} / \mathrm{vis}$ detector for the analytical ultracentrifuge, Eur. Biophys. J., 39 (2010) 397-403. [DOI: 10.1007/s00249-009-0412-6]

[166] S.H. Wu, C.Y. Mou, H.P. Lin, Synthesis of mesoporous silica nanoparticles, Chem. Soc. Rev., 42 (2013) 3862-3875. [DOI: 10.1039/c3cs35405a] 
[167] T. Itoh, K. Yano, Y. Inada, Y. Fukushima, Stabilization of chlorophyll $a$ in mesoporous silica and its pore size dependence, J. Mater. Chem., 12 (2002) 3275-3277. [DOI: 10.1039/b203923k]

[168] T. Itoh, K. Yano, Y. Inada, Y. Fukushima, Photostabilized Chlorophyllain Mesoporous Silica: Adsorption Properties and Photoreduction Activity of Chlorophyll a, J. Am. Chem. Soc., 124 (2002) 13437-13441. [DOI: 10.1021/ja0203059]

[169] T. Itoh, K. Yano, T. Kajino, S. Itoh, Y. Shibata, H. Mino, R. Miyamoto, Y. Inada, S. Iwai, Y. Fukushima, Nanoscale Organization of Chlorophyllain Mesoporous Silica: Efficient Energy Transfer and Stabilized Charge Separation as in Natural Photosynthesis, J. Phys. Chem. B, 108 (2004) 13683-13687. [DOI: 10.1021/jp047661t] [170] M.J. Llansola-Portoles, R.E. Palacios, D. Gust, T.A. Moore, A.L. Moore, Aritificial Photosynthesis: From Moleculart to Hybrid Nanoconstructs, in: E.A. Rozhkova, K. Ariga (Eds.) From Molecules to Material: Pathways to Artificial Photosynthesis, Springer, Heidelberg, New York, Dordrecht, London, 2015, pp. 71-98.

[171] R.V. Bensasson, E.J. Land, A.L. Moore, R.L. Crouch, G. Dirks, T.A. Moore, D. Gust, Mimicry of Antenna and Photoprotective Carotenoid Functions by a Synthetic Carotenoporphyrin, Nature, 290 (1981) 329-332. [DOI: 10.1038/290329a0]

[172] A.L. Moore, A. Joy, R. Tom, D. Gust, T.A. Moore, R.V. Bensasson, E.J. Land, Photoprotection by carotenoids during photosynthesis: motional dependence of intramolecular energy transfer, Science, 216 (1982) 982-984. [DOI: 10.1126/science.216.4549.982]

[173] E.J. Land, D. Lexa, R.V. Bensasson, D. Gust, T.A. Moore, A.L. Moore, P.A. Liddell, G.A. Nemeth, Pulse Radiolytic and Electrochemical Investigations of Intramolecular Electron-Transfer in Carotenoporphyrins and Carotenoporphyrin Quinone Triads, J. Phys. Chem., 91 (1987) 4831-4835. [DOI: 10.1021/j100302a036]

[174] T.A. Moore, D. Gust, P. Mathis, J.-C. Mialocq, C. Chachaty, R.V. Bensasson, E.J. Land, D. Doizi, P.A. Liddell, W.R. Lehman, G.A. Nemeth, A.L. Moore, Photodriven charge separation in a carotenoporphyrin-quinone triad, Nature, 307 (1984) 630-632. [DOI: $10.1038 / 307630 \mathrm{a} 0$ ]

[175] D. Gust, T.A. Moore, Mimicking photosynthesis, Science, 244 (1989) 35-41.

[DOI: $10.1126 /$ science.244.4900.35]

[176] R.A. Marcus, Electron Transfer Reactions in Chemistry: Theory and Experiment (Nobel Lecture), Angew. Chem. Int. Ed., 32 (1993) 1111-1121. [DOI: 
10.1002/anie.199311113]

[177] D. Gust, T.A. Moore, A.L. Moore, Mimicking Photosynthetic Solar Energy Transduction, Acc. Chem. Res., 34 (2001) 40-48. [DOI: 10.1021/ar9801301]

[178] D. Gust, T.A. Moore, A.L. Moore, Solar fuels via artificial photosynthesis, Acc. Chem. Res., 42 (2009) 1890-1898. [DOI: 10.1021/ar900209b]

[179] D. Gust, T.A. Moore, A.L. Moore, D. Barrett, L.O. Harding, L.R. Makings, P.A. Liddell, F.C. De Schryver, M. Van der Auweraer, R.V. Bensasson, M. Rougée, Photoinitiated charge separation in a carotenoid-porphyrin-diquinone tetrad: enhanced quantum yields via multistep electron transfers, J. Am. Chem. Soc., 110 (1988) 321-323. [DOI: $10.1021 / \mathrm{ja} 00209 \mathrm{a} 068$ ]

[180] D. Gust, T.A. Moore, A.L. Moore, S.J. Lee, E. Bittersmann, D.K. Luttrull, A.A. Rehms, J.M. Degraziano, X.C. Ma, F. Gao, R.E. Belford, T.T. Trier, Efficient multistep photoinitiated electron transfer in a molecular pentad, Science, 248 (1990) 199-201. [DOI: 10.1126/science.248.4952.199]

[181] G. Kodis, P.A. Liddell, L. de la Garza, P.C. Clausen, J.S. Lindsey, A.L. Moore, T.A. Moore, D. Gust, Efficient Energy Transfer and Electron Transfer in an Artificial Photosynthetic Antenna-Reaction Center Complex, J. Phys. Chem. A, 106 (2002) 2036-2048. [DOI: 10.1021/jp012133s]

[182] G. Kodis, P.A. Liddell, A.L. Moore, T.A. Moore, D. Gust, Synthesis and photochemistry of a carotene-porphyrin-fullerene model photosynthetic reaction center, J. Phys. Org. Chem., 17 (2004) 724-734. [DOI: 10.1002/poc.787]

[183] D. Kuciauskas, P.A. Liddell, S. Lin, T.E. Johnson, S.J. Weghorn, J.S. Lindsey, A.L. Moore, T.A. Moore, D. Gust, An artificial photosynthetic antenna-reaction center complex, J. Am. Chem. Soc., 121 (1999) 8604-8614. [DOI: 10.1021/ja991255j]

[184] F. D'Souza, R. Chitta, K. Ohkubo, M. Tasior, N.K. Subbaiyan, M.E. Zandler, M.K. Rogacki, D.T. Gryko, S. Fukuzumi, Corrole-fullerene dyads: formation of long-lived charge-separated states in nonpolar solvents, J. Am. Chem. Soc., 130 (2008) 14263-14272. [DOI: 10.1021/ja804665y]

[185] T. Baruah, M.R. Pederson, Density functional study on a light-harvesting carotenoid-porphyrin-C60 molecular triad, J. Chem. Phys., 125 (2006) 164706. [DOI: 10.1063/1.2360265]

[186] T. Baruah, M.R. Pederson, DFT Calculations on Charge-Transfer States of a Carotenoid-Porphyrin-C60Molecular Triad, J. Chem. Theo. Comp., 5 (2009) 834-843. 
[DOI: $10.1021 /$ ct900024f]

[187] N. Spallanzani, C.A. Rozzi, D. Varsano, T. Baruah, M.R. Pederson, F. Manghi, A. Rubio, Photoexcitation of a light-harvesting supramolecular triad: a time-dependent DFT study, J. Phys. Chem. B, 113 (2009) 5345-5349. [DOI: 10.1021/jp900820q]

[188] G. Su, A. Czader, D. Homouz, G. Bernardes, S. Mateen, M.S. Cheung, Multiscale simulation on a light-harvesting molecular triad, J. Phys. Chem. B, 116 (2012) 8460-8473. [DOI: 10.1021/jp212273n]

[189] C.A. Rozzi, S.M. Falke, N. Spallanzani, A. Rubio, E. Molinari, D. Brida, M. Maiuri, G. Cerullo, H. Schramm, J. Christoffers, C. Lienau, Quantum coherence controls the charge separation in a prototypical artificial light-harvesting system, Nat. Commun., 4 (2013) 1602. [DOI: 10.1038/ncomms2603]

[190] L.G.C. Rego, B.C. Hames, K.T. Mazon, J.-O. Joswig, Intramolecular Polarization Induces Electron-Hole Charge Separation in Light-Harvesting Molecular Triads, J. Phys. Chem. C, 118 (2014) 126-134. [DOI: 10.1021/jp408955e]

[191] A.K. Manna, D. Balamurugan, M.S. Cheung, B.D. Dunietz, Unraveling the Mechanism of Photoinduced Charge Transfer in Carotenoid-Porphyrin-C60Molecular Triad, J. Phys. Chem. Lett., 6 (2015) 1231-1237. [DOI: 10.1021/acs.jpclett.5b00074]

[192] M. Cho, Coherent Two-Dimensional Optical Spectroscopy, Chem. Rev., 108 (2008) 1331-1418. [DOI: 10.1021/cr078377b]

[193] M. Cho, Two-Dimensional Optical Spectroscopy, CRC Press2009.

[194] A. Tomita, T. Sato, K. Ichiyanagi, S. Nozawa, H. Ichikawa, M. Chollet, F. Kawai, S.Y. Park, T. Tsuduki, T. Yamato, S.Y. Koshihara, S. Adachi, Visualizing breathing motion of internal cavities in concert with ligand migration in myoglobin, Proc. Natl. Acad. Sci. USA, 106 (2009) 2612-2616. [DOI: 10.1073/pnas.0807774106]

[195] C. Behrens, F.J. Decker, Y. Ding, V.A. Dolgashev, J. Frisch, Z. Huang, P. Krejcik, H. Loos, A. Lutman, T.J. Maxwell, J. Turner, J. Wang, M.H. Wang, J. Welch, J. Wu, Few-femtosecond time-resolved measurements of X-ray free-electron lasers, Nat. Commun., 5 (2014) 3762. [DOI: 10.1038/ncomms4762]

[196] K. Hirata, K. Shinzawa-Itoh, N. Yano, S. Takemura, K. Kato, M. Hatanaka, K. Muramoto, T. Kawahara, T. Tsukihara, E. Yamashita, K. Tono, G. Ueno, T. Hikima, H. Murakami, Y. Inubushi, M. Yabashi, T. Ishikawa, M. Yamamoto, T. Ogura, H. Sugimoto, J.R. Shen, S. Yoshikawa, H. Ago, Determination of damage-free crystal structure of an X-ray-sensitive protein using an XFEL, Nat. Methods, 11 (2014) 734-736. [DOI: 
10.1038/nmeth.2962]

[197] D. Arnlund, L.C. Johansson, C. Wickstrand, A. Barty, G.J. Williams, E. Malmerberg, J. Davidsson, D. Milathianaki, D.P. DePonte, R.L. Shoeman, D. Wang, D. James, G. Katona, S. Westenhoff, T.A. White, A. Aquila, S. Bari, P. Berntsen, M. Bogan, T.B. van Driel, R.B. Doak, K.S. Kjaer, M. Frank, R. Fromme, I. Grotjohann, R. Henning, M.S. Hunter, R.A. Kirian, I. Kosheleva, C. Kupitz, M. Liang, A.V. Martin, M.M. Nielsen, M. Messerschmidt, M.M. Seibert, J. Sjohamn, F. Stellato, U. Weierstall, N.A. Zatsepin, J.C. Spence, P. Fromme, I. Schlichting, S. Boutet, G. Groenhof, H.N. Chapman, R. Neutze, Visualizing a protein quake with time-resolved X-ray scattering at a free-electron laser, Nat. Methods, 11 (2014) 923-926. [DOI: 10.1038/nmeth.3067]

[198] Y. Kurashige, H. Nakano, Y. Nakao, K. Hirao, The $\pi \rightarrow \pi *$ excited states of long linear polyenes studied by the CASCI-MRMP method, Chem. Phys. Lett., 400 (2004) 425-429. [DOI: 10.1016/j.cplett.2004.10.141]

[199] J.B.L. Martins, J.A. Duraes, M.J.A. Sales, A.F.A. Vilela, G.M.E. Silva, R. Gargano, Theoretical Investigation of Carotenoid Ultraviolet Spectra, Int. J. Quantum Chem., 109 (2009) 739-745. [DOI: 10.1002/qua.21845]

[200] M. Kleinschmidt, C.M. Marian, M. Waletzke, S. Grimme, Parallel multireference configuration interaction calculations on mini- $\beta$-carotenes and $\beta$-carotene, J. Chem. Phys., 130 (2009) 044708. [DOI: 10.1063/1.3062842] 


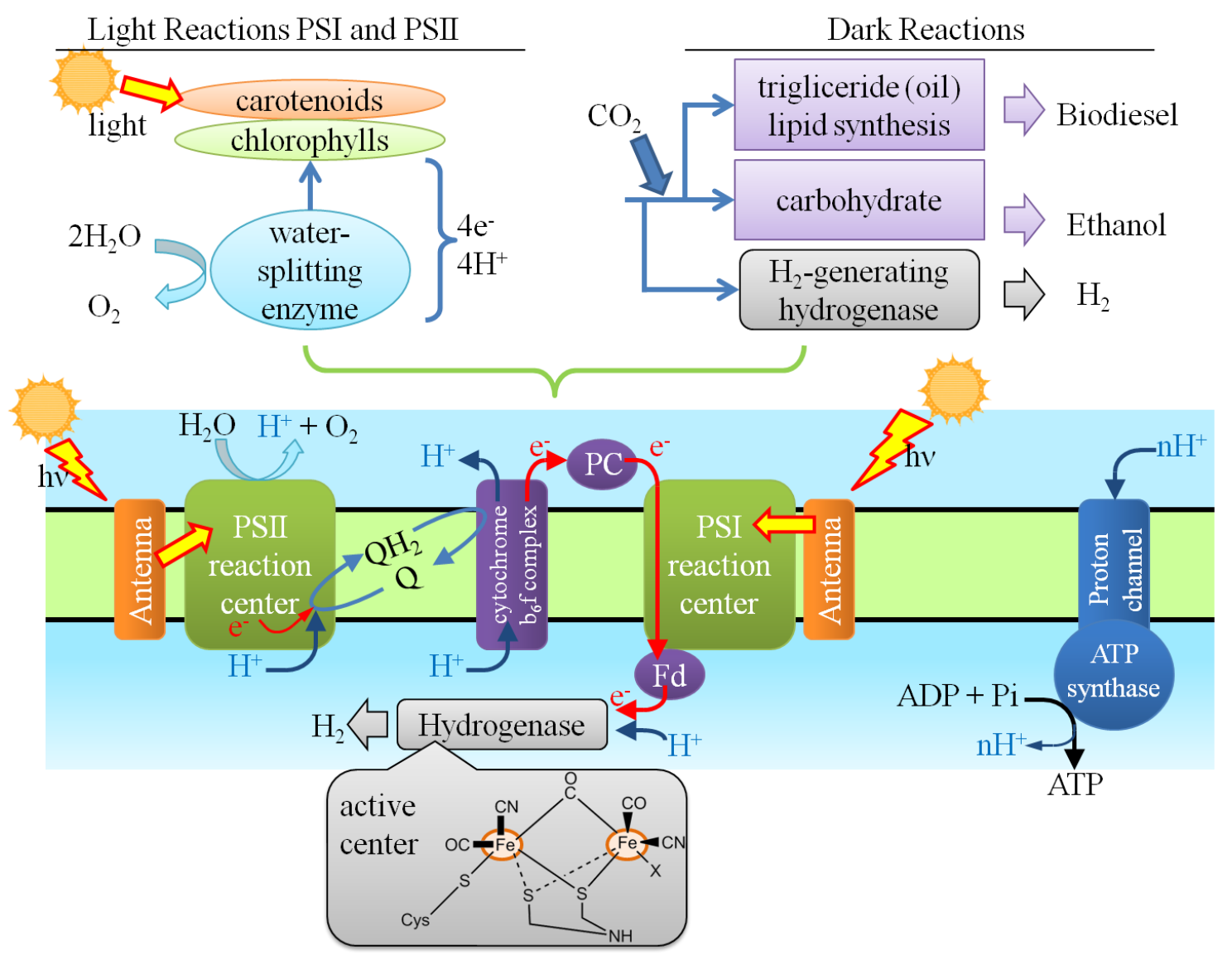

Figure 1. A schematic description of light energy conversion processes by oxygenic photosynthesis organisms in the photosynthetic membrane. (Rearranged from reference [8] with permission) 


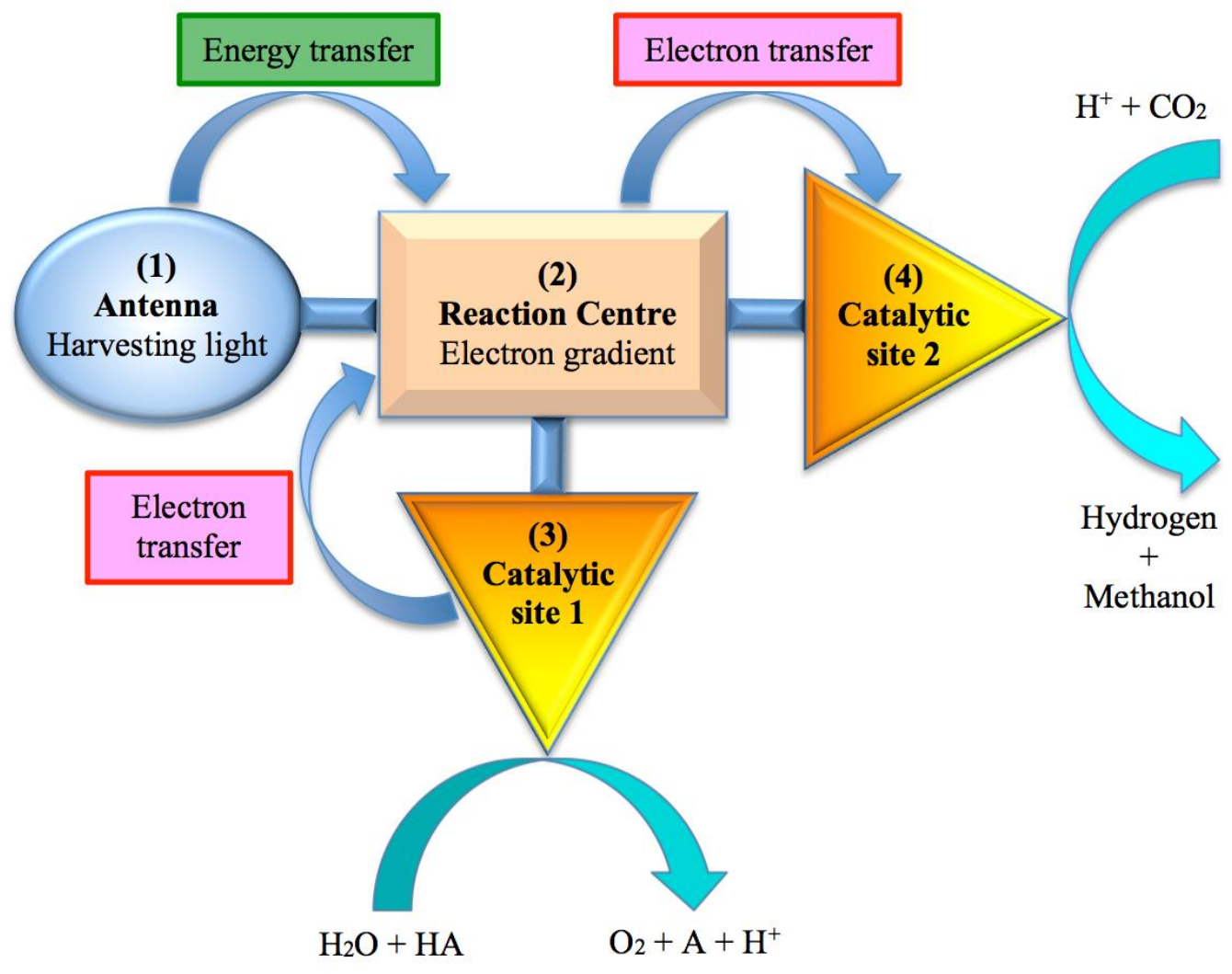

Figure 2. A schematic description that shows the principles of artificial photosynthesis (Artificial Leaf). Whole process can be divided into four elements; (1) light-harvesting, (2) reaction centre, (3) oxidation catalyst, and (4) reduction catalyst. 


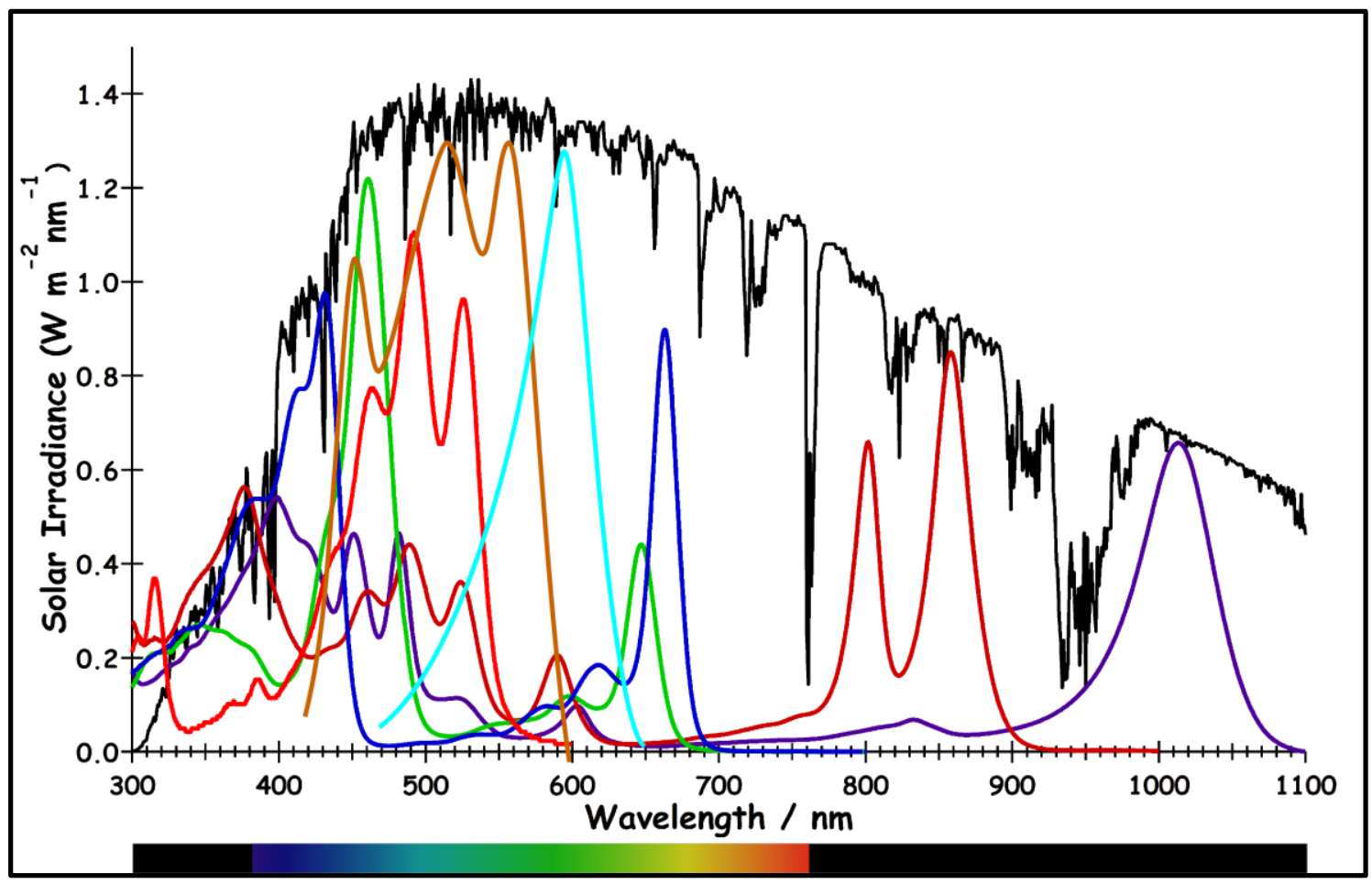

Figure 3. The spectrum of solar radiation (black solid line) and the absorption spectra of light-harvesting antenna pigments and pigment-protein complexes from various photosynthetic organisms (blue, chlorophyll $a$; green, chlorophyll $b$; red, $\beta$-carotene; brown, phycoerythrin; cyan, phycocyanin; dark-brown, LH2 complex from Rba. sphaeroides 2.4.1; purple, LH1-RC complex from Blc. viridis). The photosynthetic antennas that nature had created cover up perfectly all the wavelengths range of the solar radiation. (Courtesy of Assoc. Prof. Ritsuko Fujii of Osaka City University.) 


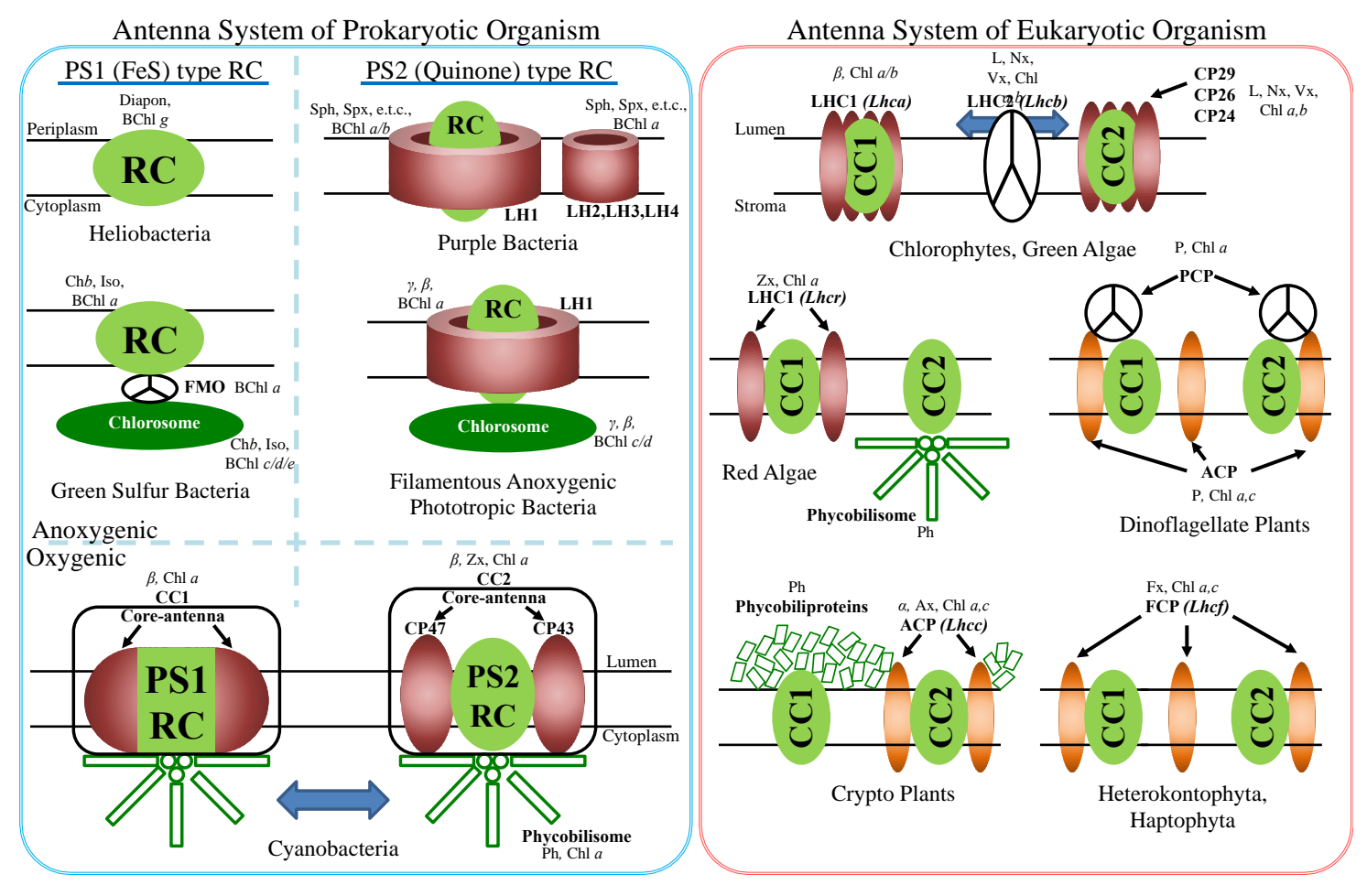

Figure 4. Schematic illustrations of antenna systems from prokaryotes and eukaryotes (Modified from the reference [3]). The ellipses display the protein of LHC super family, and their genes are written in the parentheses. The complexes that function with trimer unit are described with parting line in the circle. The complexes that proceed the state transition due to the light condition is described with the two-way arrows. Abbreviations stand for as follows. $\alpha$ : $\alpha$-carotene; $\beta$ : $\beta$-carotene; $\gamma$ : $\gamma$-carotene; Ax: Alloxanthin; Bchl: Bacteriochlorophyll; Chb; Chlorobactene; Chl: Chlorophyll; Diapon: Diaponeurosporene; Fx: Fucoxanthin; Iso: Isorenieratene; L: Lutein; Nx: Neoxanthin; P: Peridinin; Ph: Phycobiline; Sph: Spheroidene; Spx: Spirilloxanthin; Vx: Violaxanthin; Zx: Zeaxanthin; CC1,CC2: Core Complex 1,2; RC: Reaction Centre; PSI, II: Photosystem I, II; PCP: Peridinin-Chlorophyll $a$ binding Protein; ACP: Chlorophyll $a / c$ Protein; FCP: Fucoxanthin- Chlorophyll a/c binding Protein; LH1, LH2, LH3, LH4: Light Harvesting Antenna 1,2,3,4. 


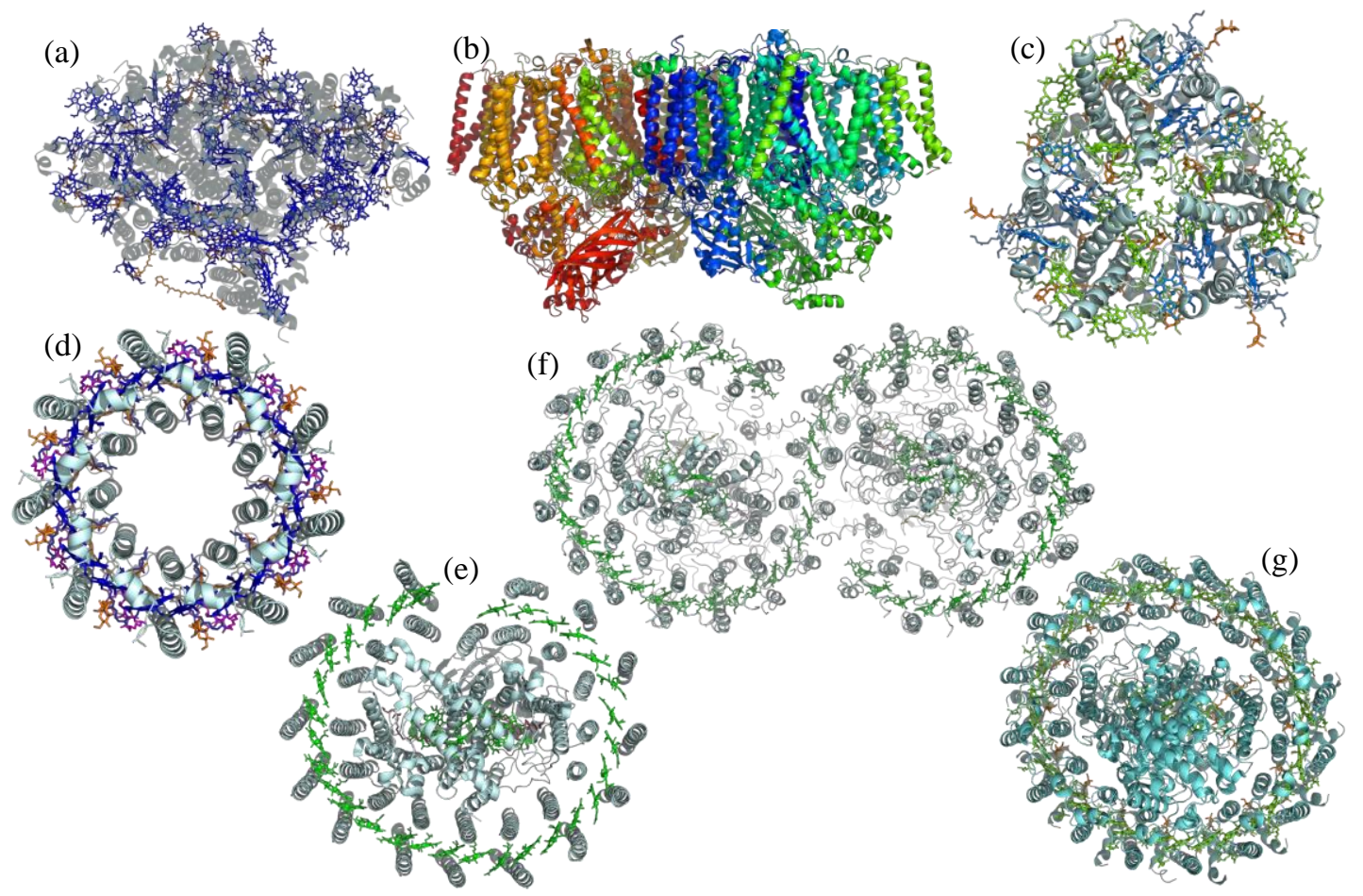

Figure 5. High resolution crystallographic structures of (a) PS I (PDB: 1JB0) and (b) PS II (3WU2) from cyanobacteria, (c) LHCII (1RWT) from higher plants, (d) LH2 (1KZU) from a purple photosynthetic bacterium Rbl. acidophilus 10050, and RC-LH1 core complexes from (e) Rps. palustris (1PYH), (f) Rba. sphaeroides (4V9G), and (g) Thermochromatium tepidum (4V8K), which are drawn using PyMol software with data from protein databank (PDB). Carotenoids are bound to all these complexes nearby chlorophyll or bacteriochlorophyll molecules. 


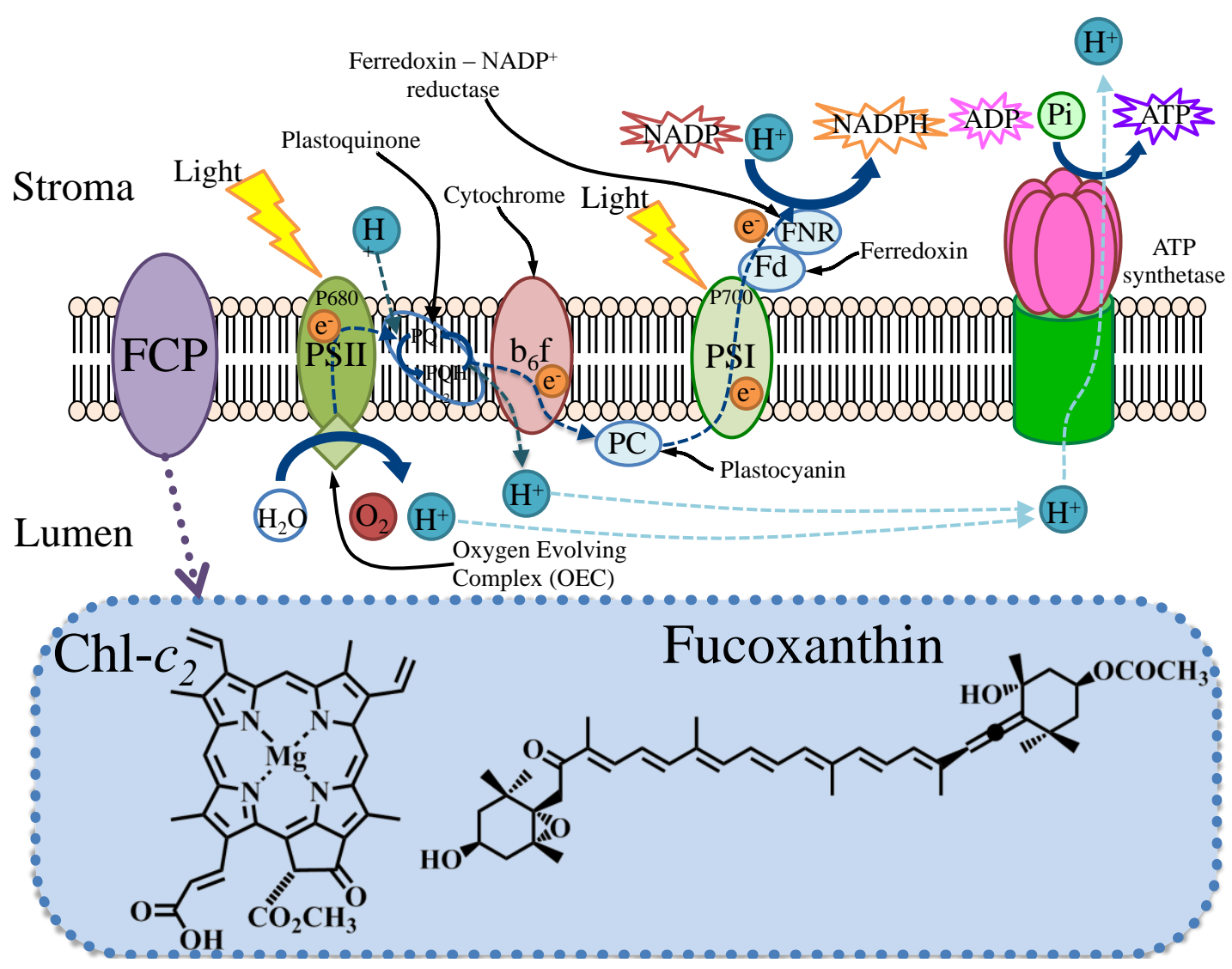

Figure 6. A schematic illustration that shows the energy, electron and proton $\left(\mathrm{H}^{+}\right)$flows in thylakoids membranes of marine algae. 


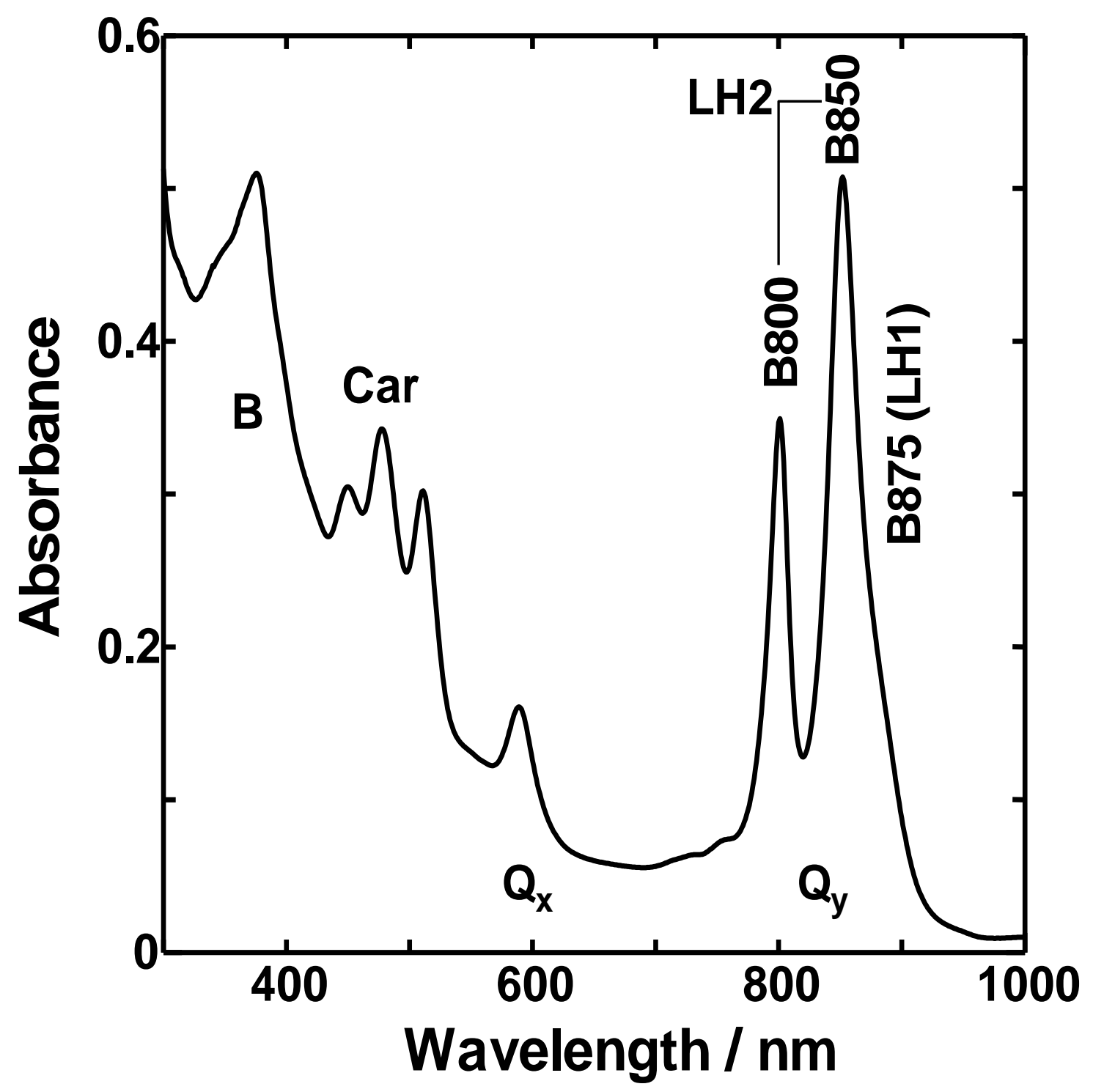

Figure 7. Absorption spectrum of chromatophores (photosynthetic membranes) from a purple photosynthetic bacterium Rba. sphaeroides 2.4.1 recorded at room temperature in Tris- $\mathrm{HCl}(\mathrm{pH}=8.0)$ buffer solution. The abbreviations stand for as follows: Car, absorption band of carotenoid (spheroidene); B, Soret absorption band of bacteriochlorophyll (Bchl) a; $\mathrm{Q}_{\mathrm{x}}, \mathrm{Q}_{\mathrm{x}}$ absorption band of $\mathrm{Bchl} a ; \mathrm{Q}_{\mathrm{y}}, \mathrm{Q}_{\mathrm{y}}$ absorption band of $\mathrm{Bchl} a$; B800, B800 absorption band of monomeric array of Bchl $a$ bound to LH2 complex; B850, B850 absorption band of dimeric array of Bchl $a$ bound to LH2 complex; B875, B875 absorption band of dimeric array of Bchl $a$ bound to LH1 complex. 
(a)<smiles>COC(C)(C)C/C=C/C(C)=C/C=C/C(C)=C/C=C/C(C)=C/C=C/C=C(C)/C=C/C=C(\C)CC/C=C(\C)CCC=C(C)C</smiles>

(b)

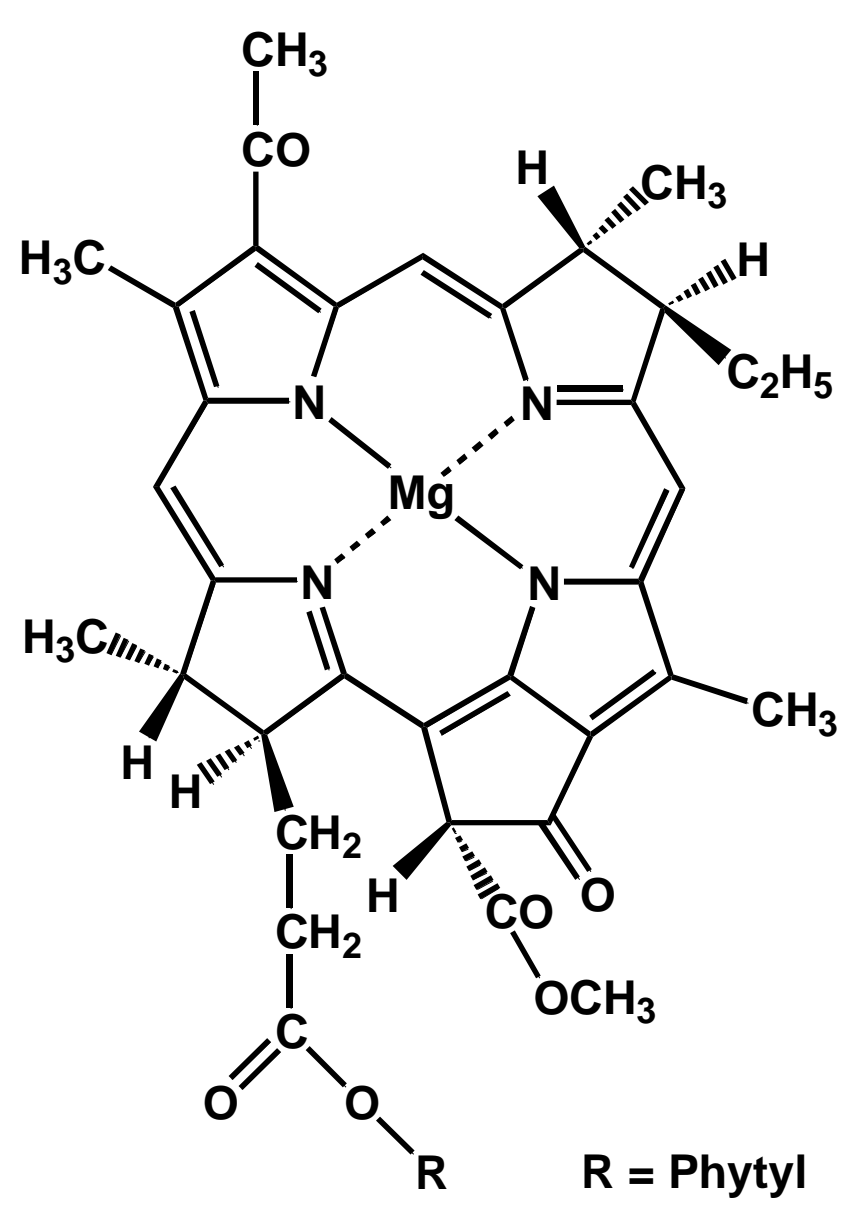

Figure 8. Chemical structures of (a) spheroidene and (b) bacteriochlorophyll $a$. 


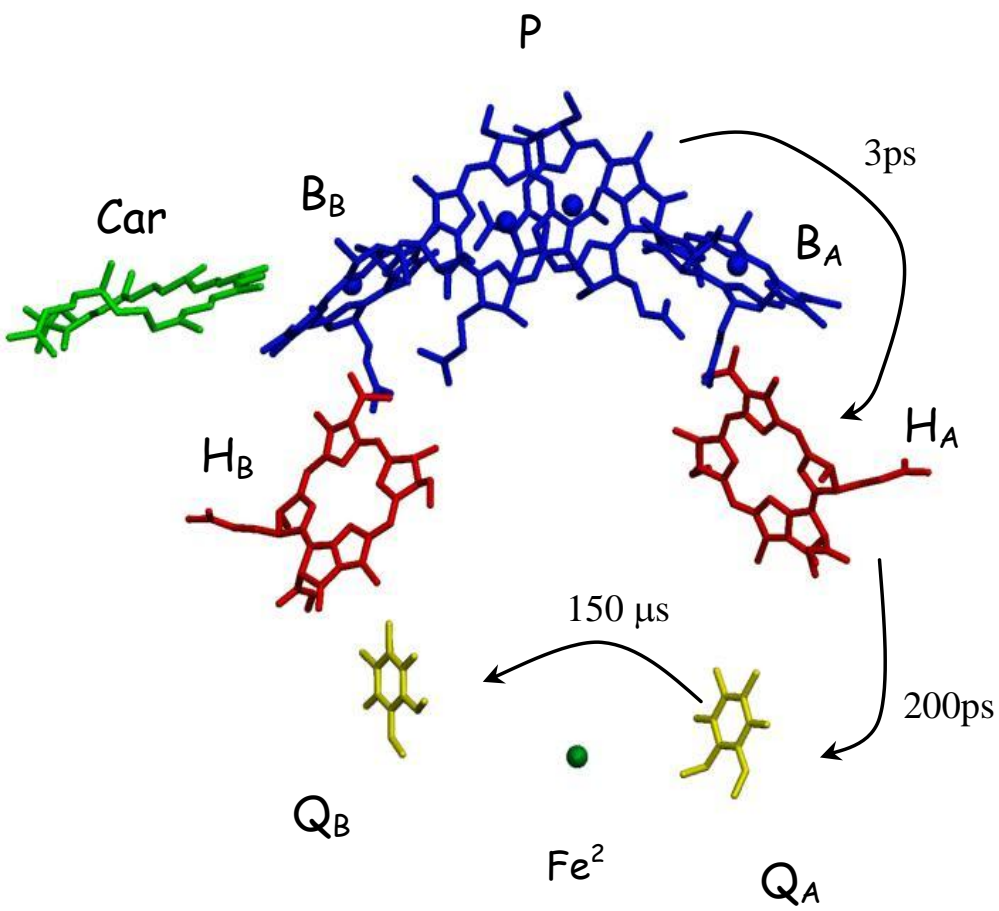

Figure 9. Pigment arrangement and the electron transfer pathway in the reaction centre from a purple photosynthetic bacterium Rba. sphaeroides, which is drawn with PDB data (1RGN) using VMD software. 


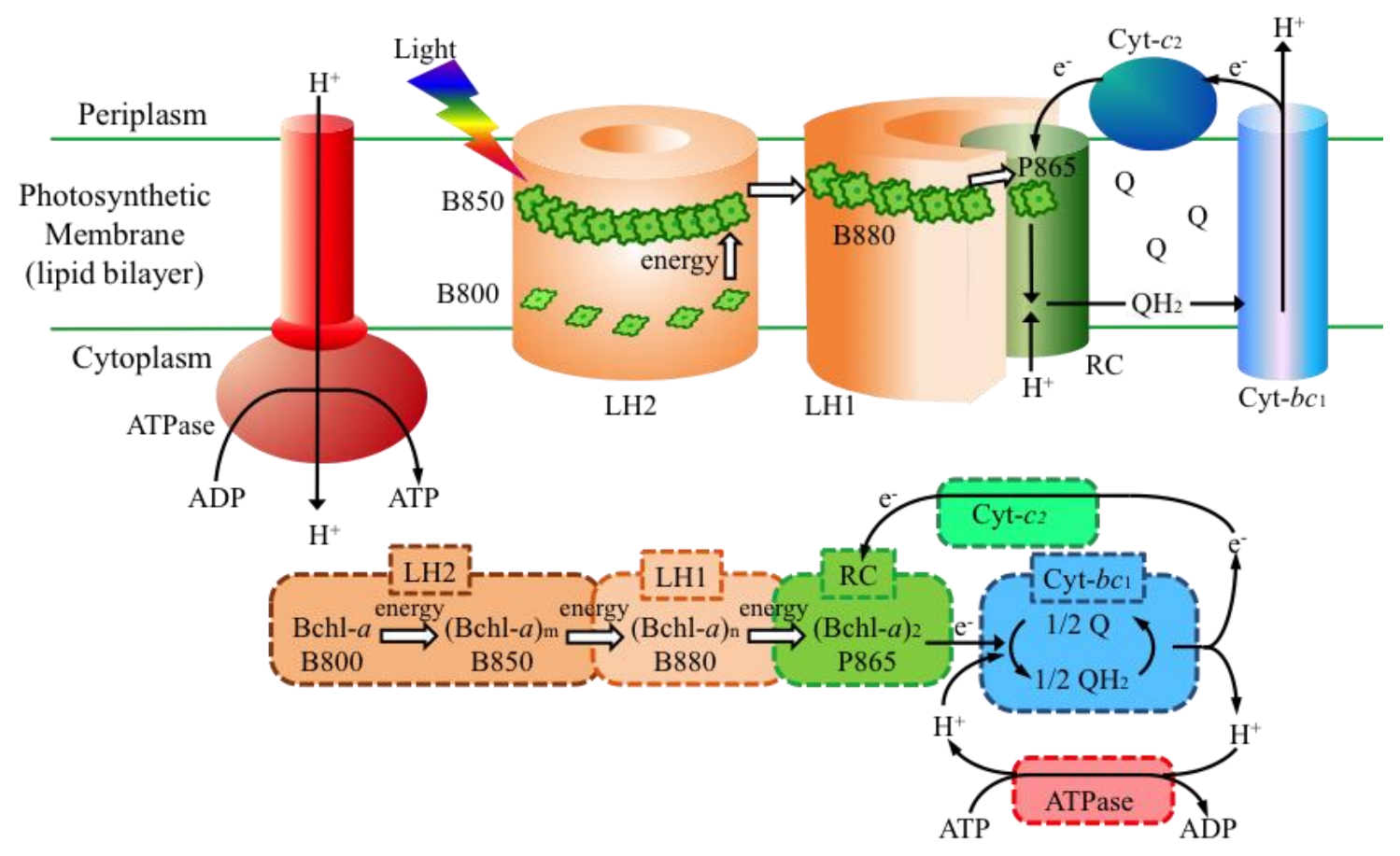

Figure 10. Schematic illustration that shows the flows of excitation energy, electron, and proton $\left(\mathrm{H}^{+}\right)$in the photosynthetic membrane from purple photosynthetic bacteria. 


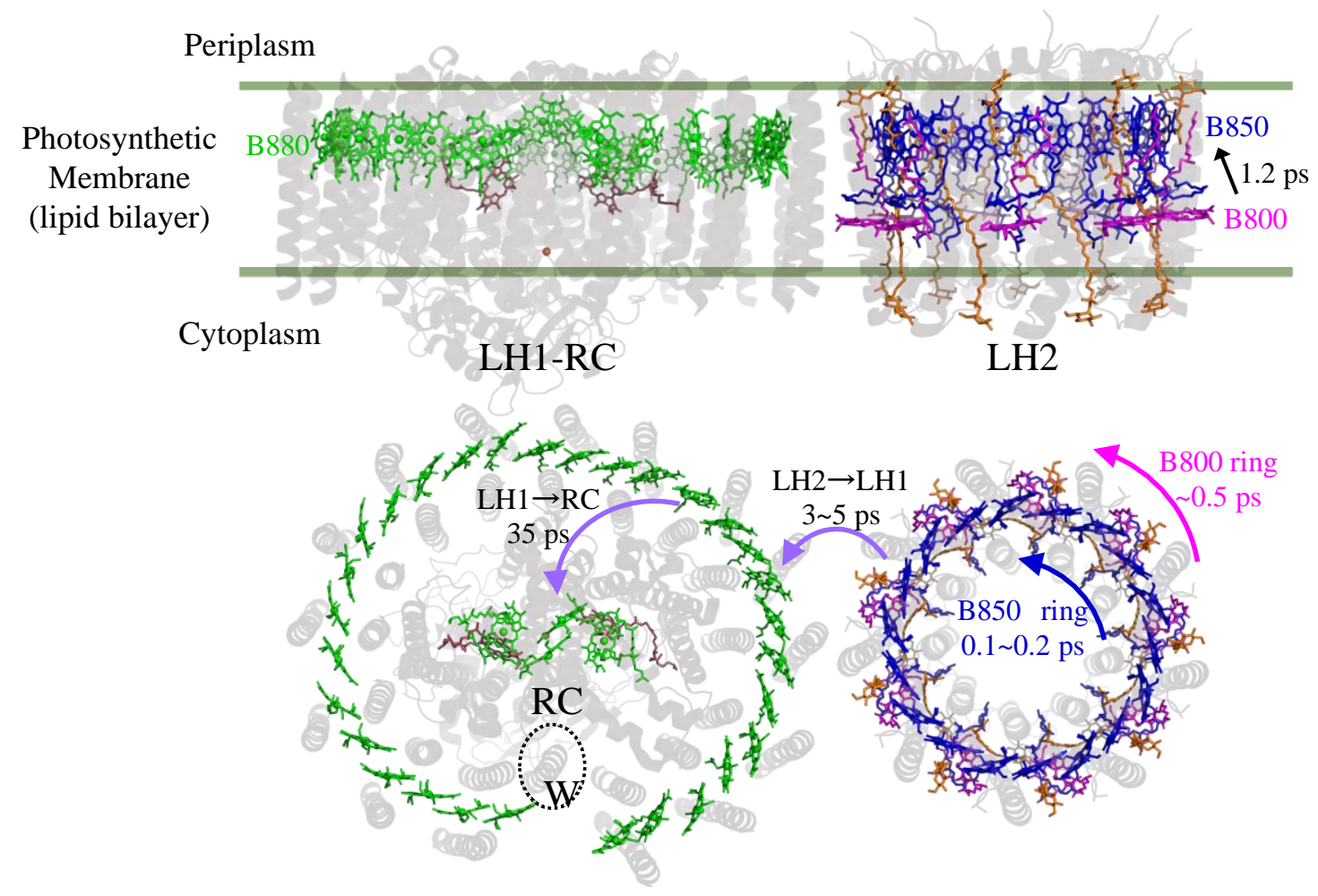

Figure 11. Structures of LH2 complex (right-hand side) from a purple photosynthetic bacterium Rbl. acidophilus 10050 (PDB: 1KZU) and those of RC-LH1 core complex (left-hand side) from Rps. palustris (PDB: 1PYH), which are drawn using PyMol software. The upper panel shows a viewgraph from horizontal direction of the photosynthetic membrane, and the lower panel shows that from the cytoplasmic side. 

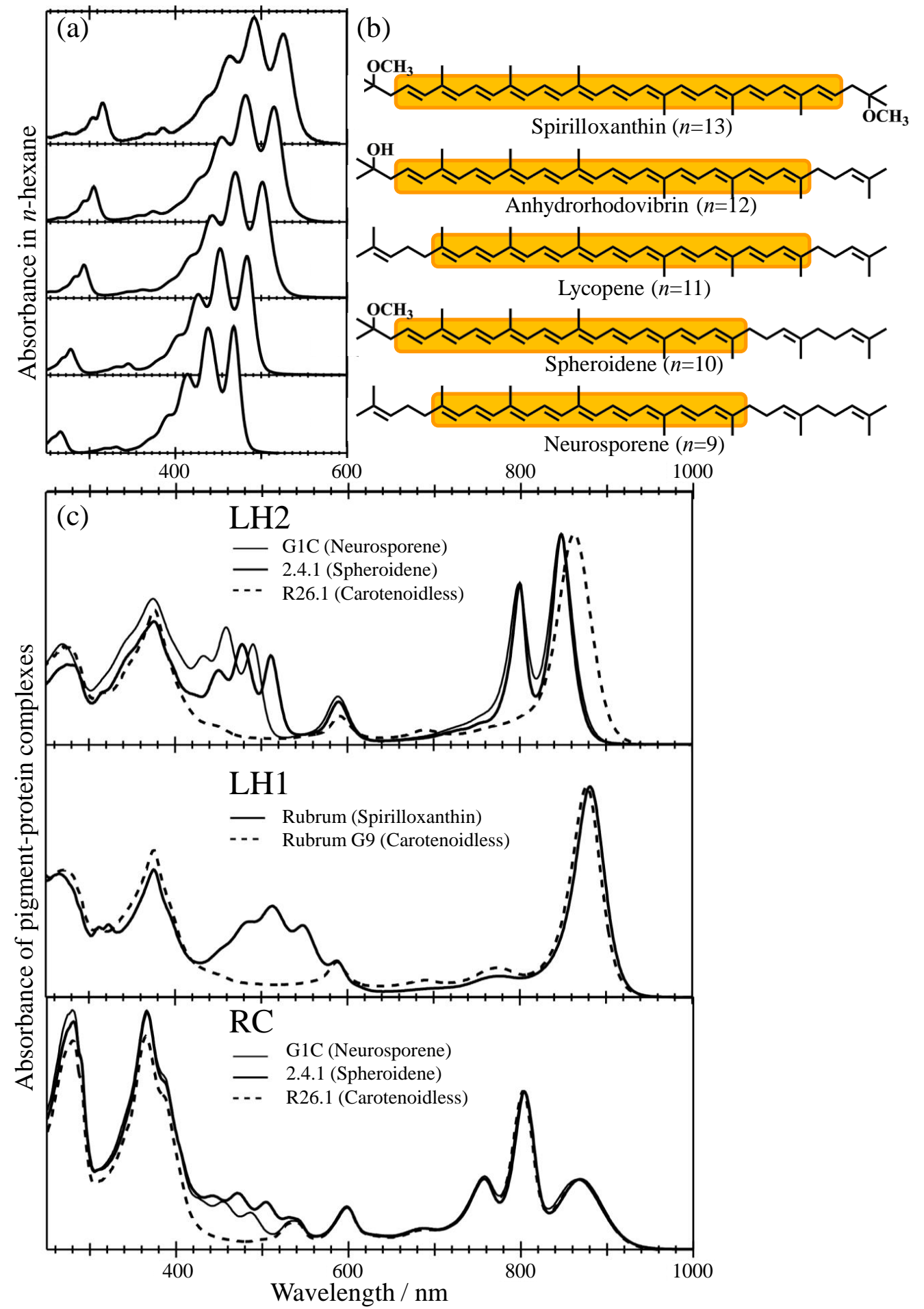

Figure 12. (a) Absorption spectra in $n$-hexane solutions and (b) chemical structures of carotenoids bound to purple photosynthetic bacteria. Absorption spectra of pigment-protein complexes in buffer solutions are shown in (c). 

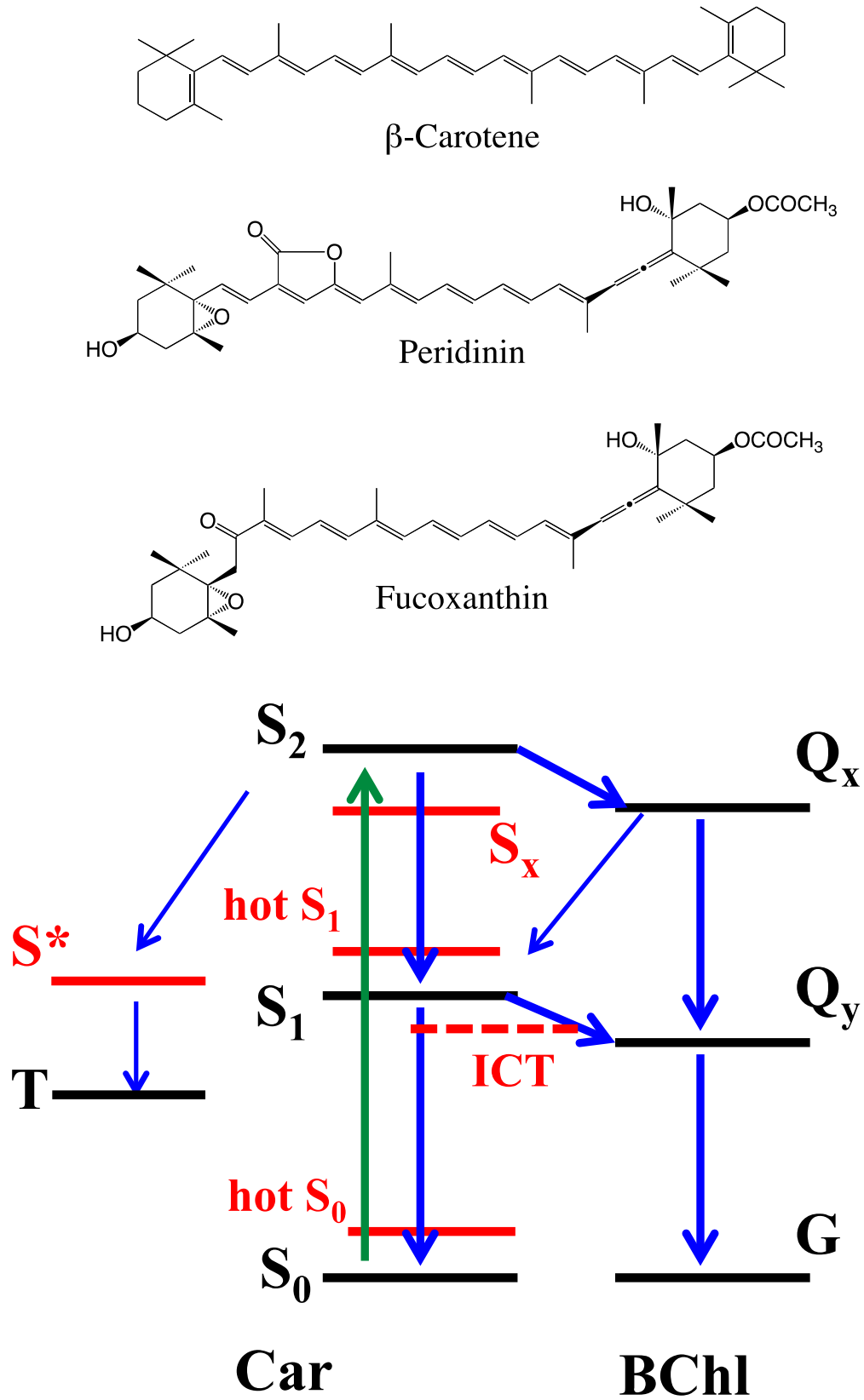

Figure 13. Chemical structures of $\beta$-carotene, peridinin, and fucoxanthin, and a schematic description of energy diagrams together with relaxation and energy-transfer pathways of carotenoids following photoexcitation up to the $S_{2}$ state. 


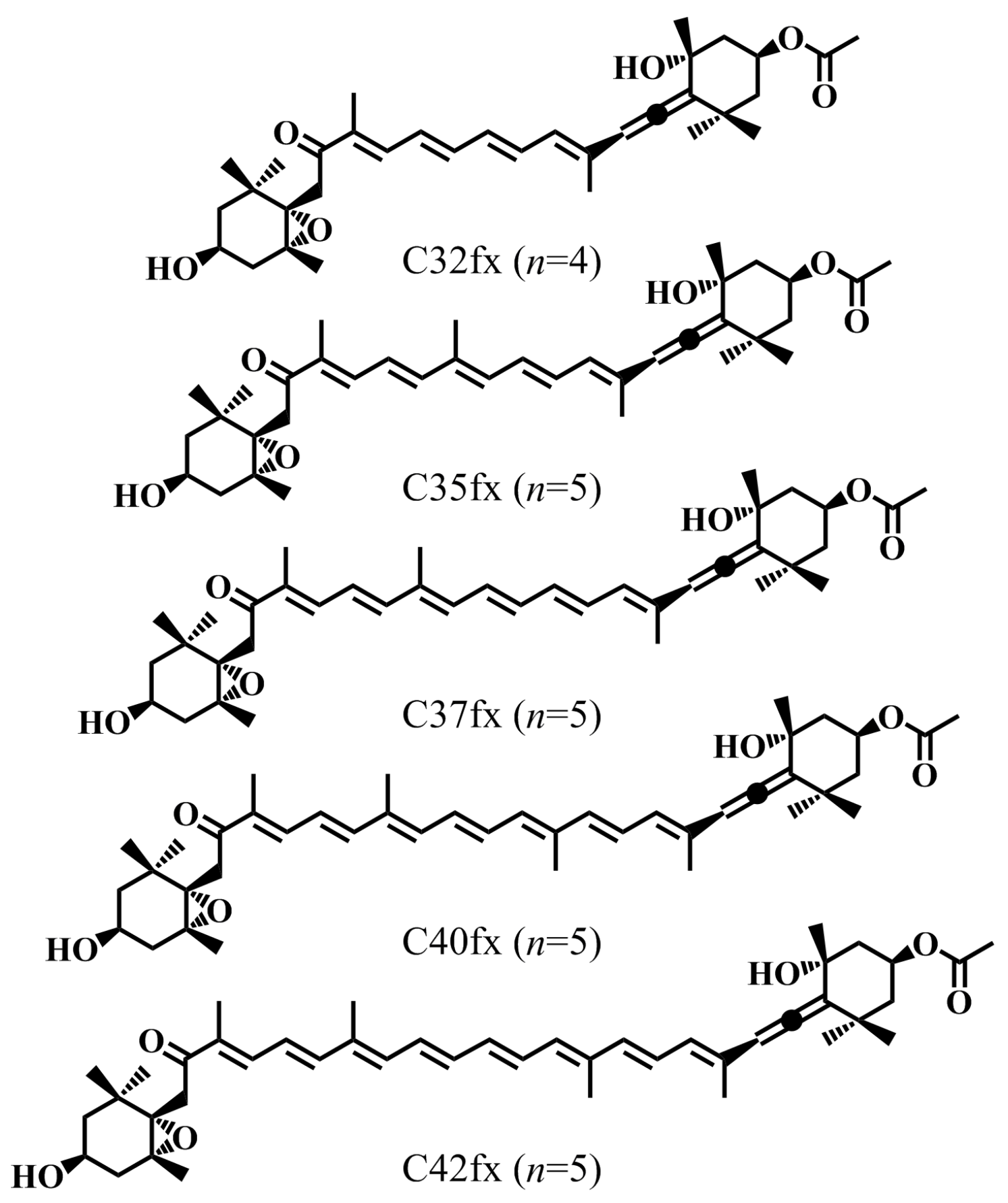

Figure 14: Chemical structures of fucoxanthin homologues. Fucoxanthin corresponds to $\mathrm{C} 40 \mathrm{fx}(n=7)$. 


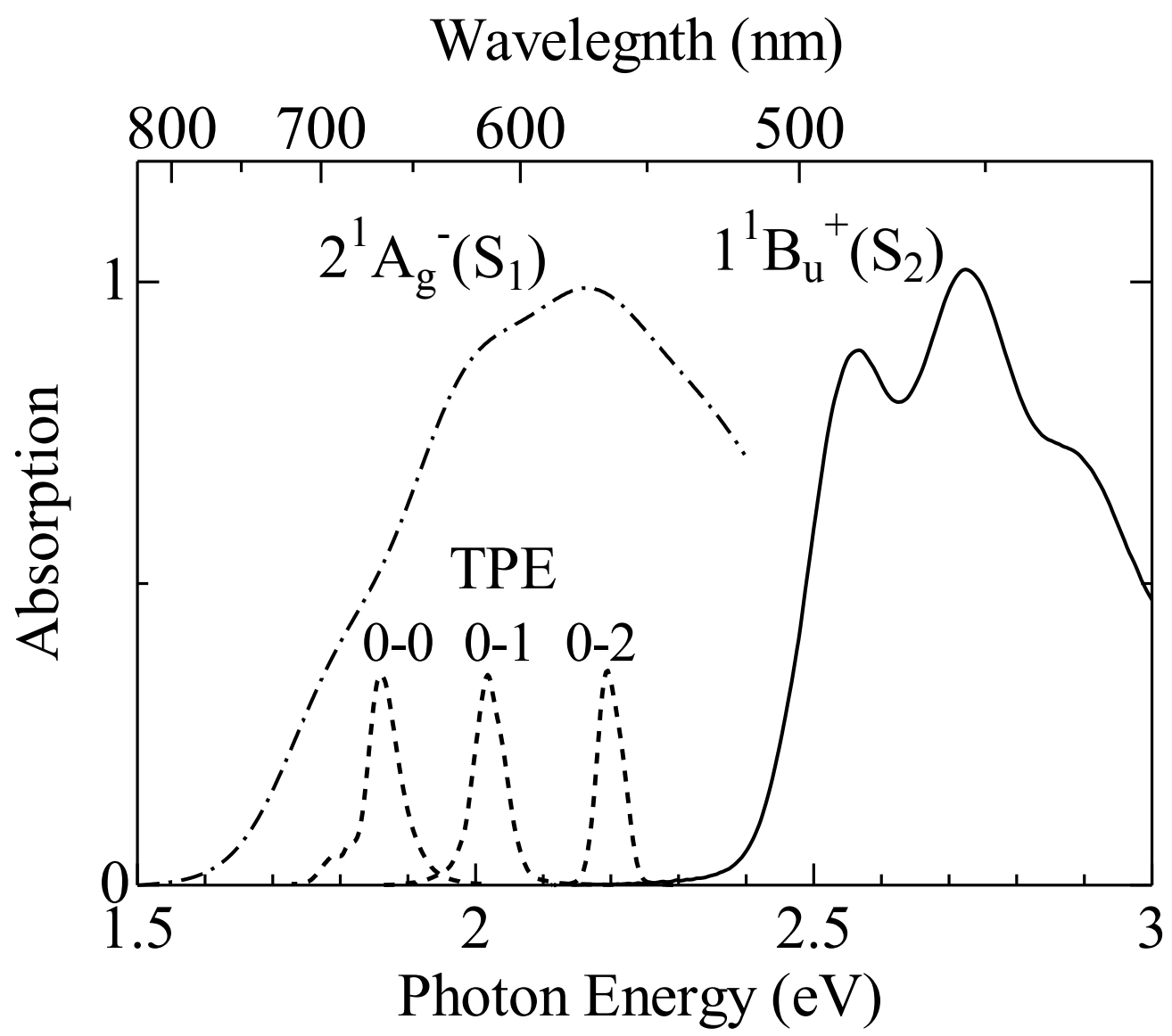

Figure 15. Steady state absorption spectra of $\beta$-carotene in cyclohexane that corresponds to the transition from the ground $1{ }^{1} \mathrm{~A}_{\mathrm{g}}{ }^{-}\left(\mathrm{S}_{0}\right)$ state to the $1{ }^{1} \mathrm{~B}_{\mathrm{u}}{ }^{+}\left(\mathrm{S}_{2}\right)$ state (solid line) and that to the $2^{1} \mathrm{~A}_{\mathrm{g}}{ }^{-}\left(\mathrm{S}_{1}\right)$ state (dotted broken line). The latter spectrum was predicted based on a theoretical calculation. The bands illustrated with broken lines are the spectra of excitation laser light sources that were used for two-photon excitation (TPE). The energies of these spectra were doubled from the original values. 


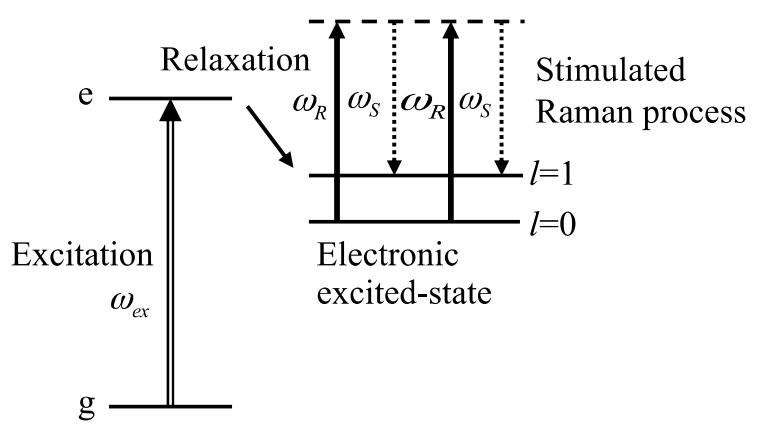

Figure 16. The outline of time-resolved Raman spectroscopy using the process of stimulated Raman scattering. 


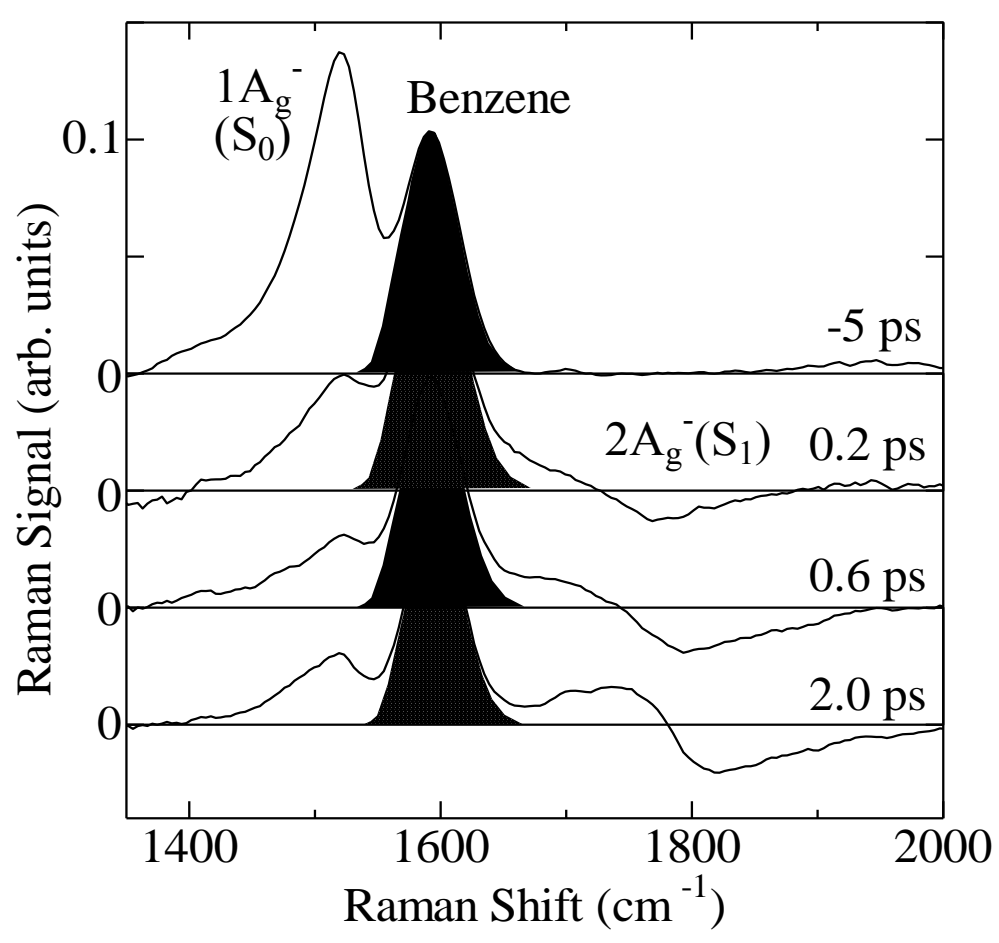

Figure 17. Time-resolved stimulated Raman spectra of $\beta$-carotene in benzene following photoexcitation. Shadowed bands show the Raman lines of the solvent. 


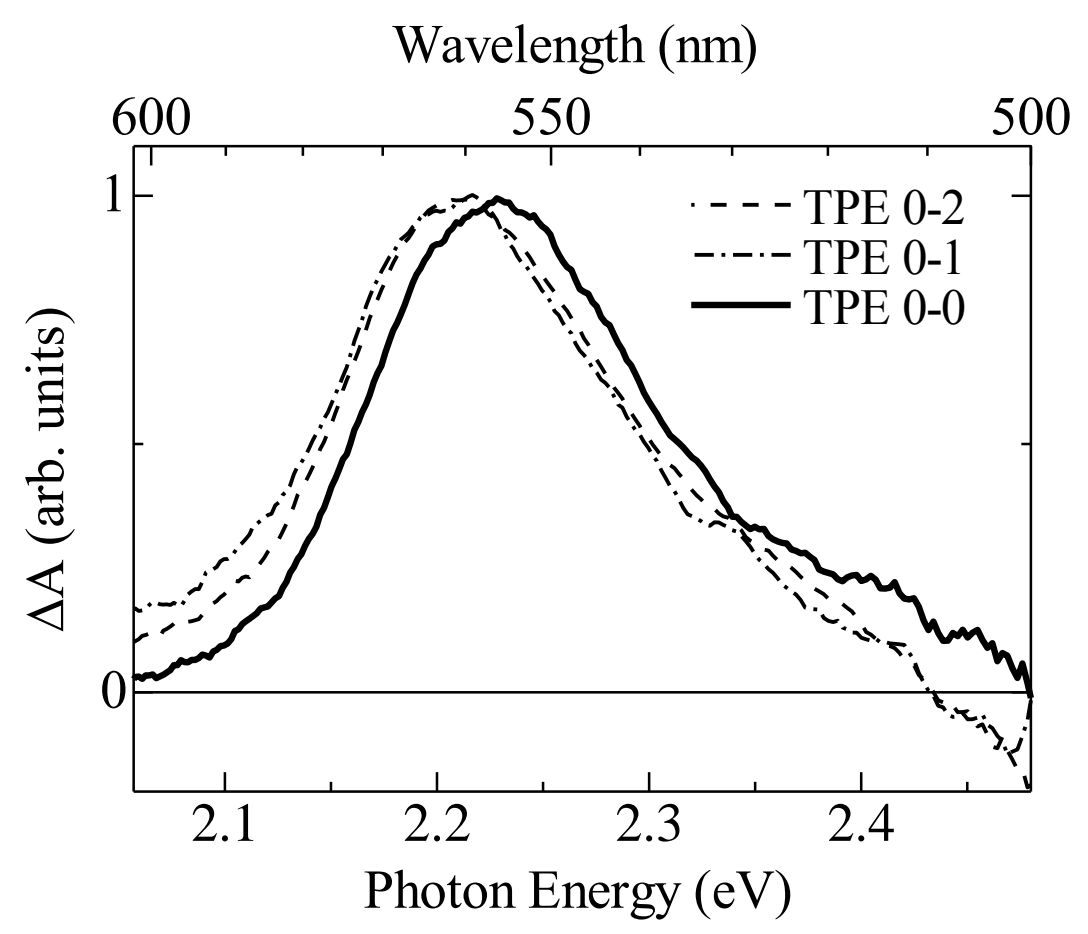

Figure 18. Transient absorption spectra of $\beta$-carotene in cyclohexane recorded at $5 \mathrm{ps}$ after two-photon excitation to the $2^{1} \mathrm{~A}_{\mathrm{g}}{ }^{-}\left(\mathrm{S}_{1}\right)$ state. 


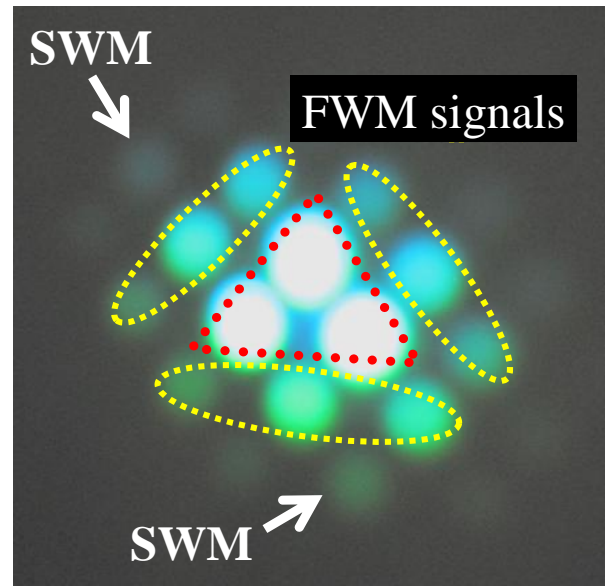

Figure 19. A photograph of four-wave mixing (FWM) signals. Central three bright spots are excitation laser lights. FWM signals surrounded by dotted ellipsoids can readily be observed with naked eyes. Six-wave mixing (SWM) signals that are generated through higher order nonlinear interaction can also be observed. 
(a)

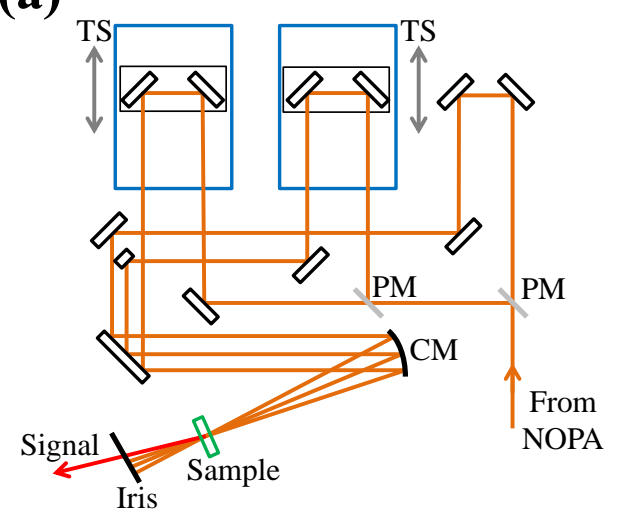

(b) $k_{1}+k_{2}-k_{3} \quad-k_{1}+k_{2}+k_{3}$
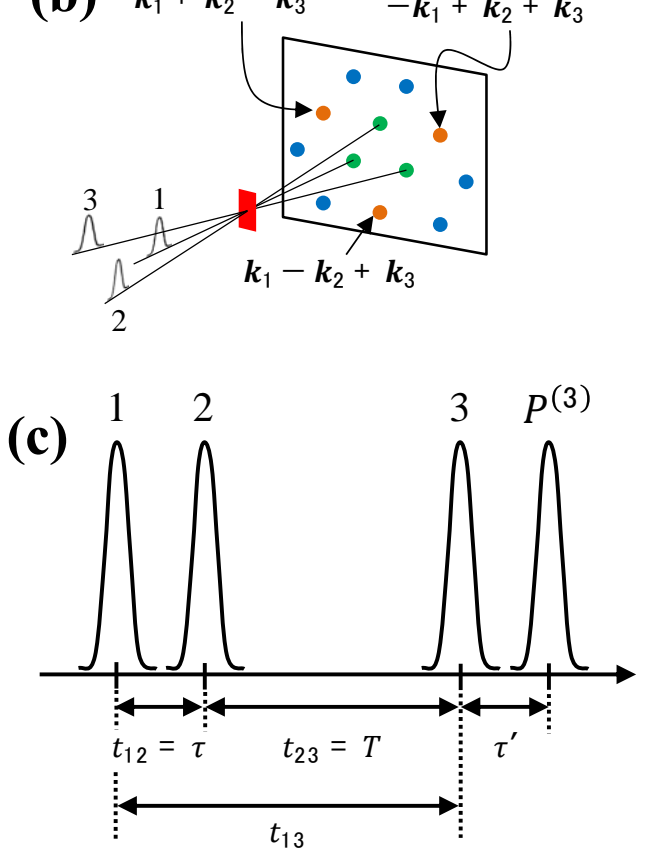

Figure 20. (a) Optical configuration of the interferometer used for the measurements of four-wave mixing (FWM) signals. The light from a non-collinearly phase-matched optical parametric amplifier (NOPA) is split into three using two pellicle mirrors (PM). Three laser pulses thus produced are then focused to excite the sample using a collimating mirror (CM). Time intervals between each of these three laser pulses were controlled using two translational stages (TS). The FWM signals are generated along the directions that satisfy the phase-match condition. One of these signals is selected using an iris diaphragm. (b) When three laser pulses are irradiated to the sample, the FWM signals can be generated along the directions that satisfy the phase-match conditions. (c) The relation between coherent time $\tau$ and population time T. $_{12}\left(t_{13}\right)$ is the time interval between pulses 1 and 2 (1and 3) when they arrive at the sample. 


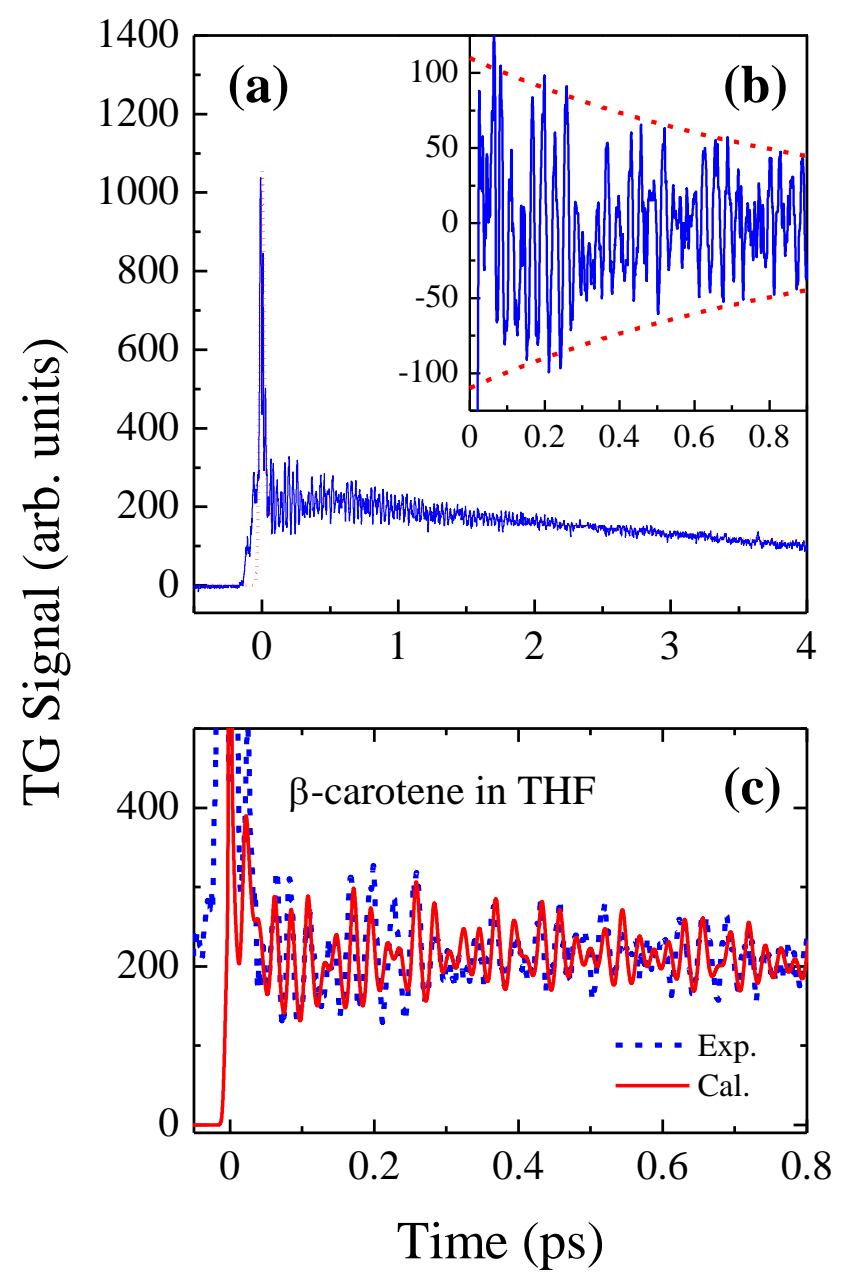

Figure 21. (a) Experimentally observed time-evolution of the transient-grating (TG) signal. A slowly varying background shown with dotted lines reflects the lifetime of the electronic excited states. If the background is subtracted from the original TG signal, coherent vibration component can be extracted as shown in (b). The decay time of coherent vibration is determined to be around 1 ps. (c) Comparison of the experimentally observed TG signal (broken line) and the result of theoretical calculation (solid line). 


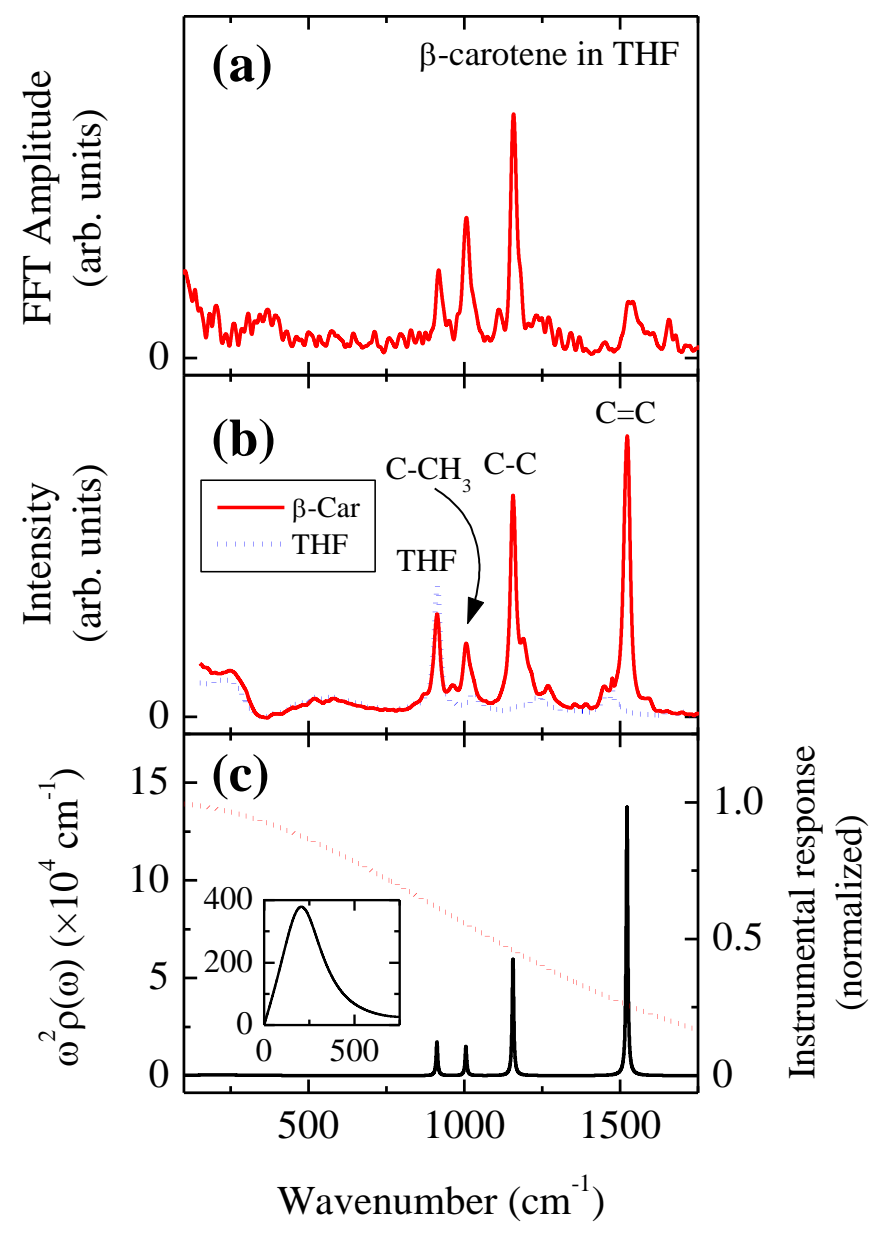

Figure 22. (a) Fourier transformed spectrum of the coherent signal component shown in Figure 21(b). (b) Raman spectra of $\beta$-carotene and solvent THF (tetrahydrofuran). (c) Spectral density (solid line) and the response function of the detecting system (dotted line). The inset shows the spectral density that reflects the system-bath ( $\beta$-carotene and solvent THF) interaction in the low-frequency regime. 
(a)

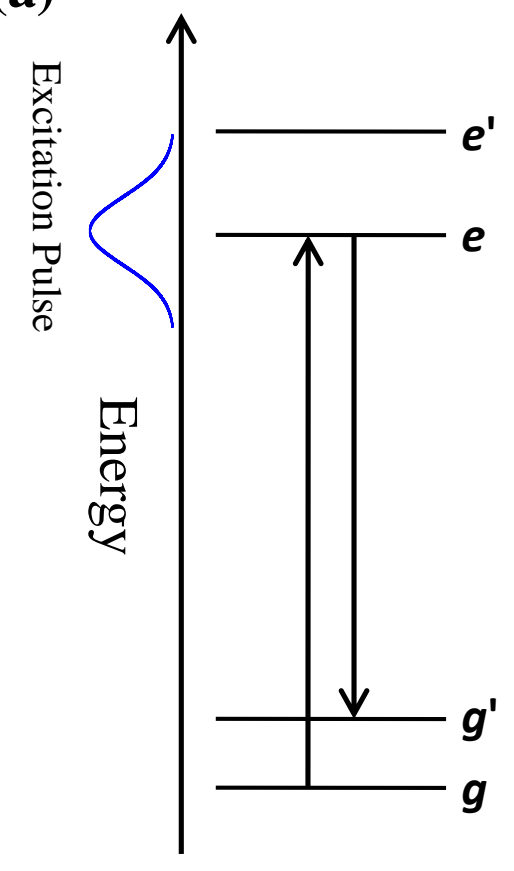

(b)

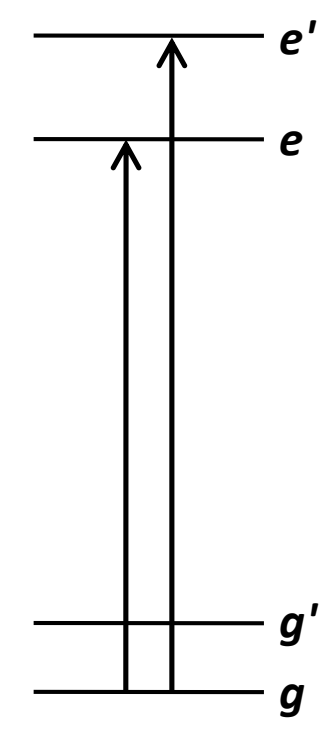

Figure 23. Coherent vibration (a) in the ground state and (b) in the excited state induced by the exposure of two excitation laser pulses $\left(-\boldsymbol{k}_{1}\right.$ and $\left.\boldsymbol{k}_{2}\right)$. The spectral bandwidth of the excitation pulse should exceed the energy difference of the vibrational levels. 

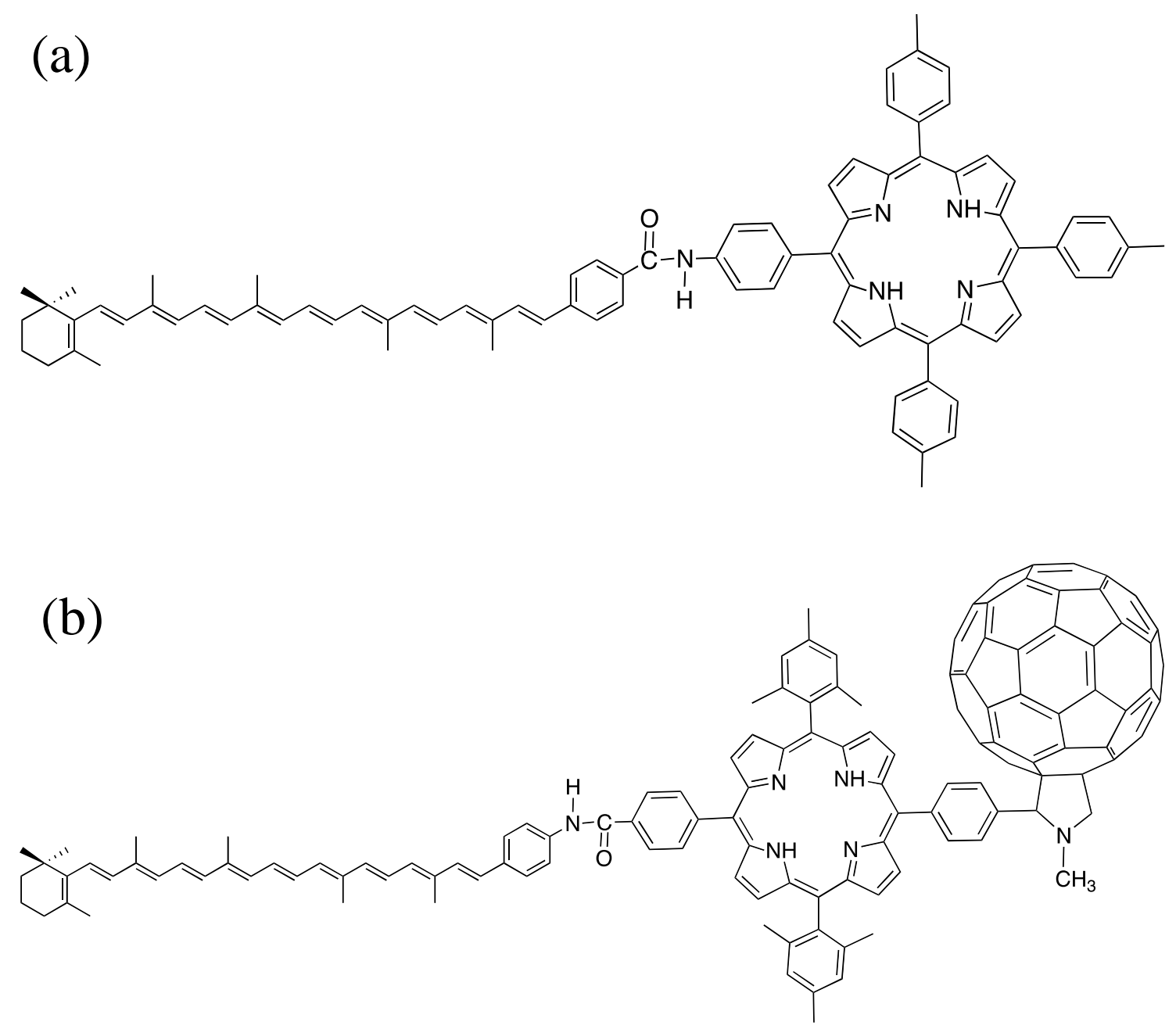

Figure 24. Chemical structures of (a) Carotenoid(C)-Porphyrin(P) dyad (b) C-P-Fullerene $\left(\mathrm{C}_{60}\right)$ triad. 


\section{Author Biography and Photography}

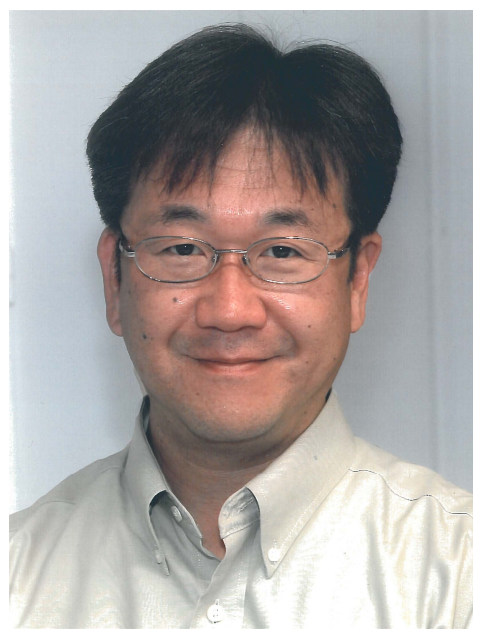

Hideki Hashimoto was born in 1962 in Kyoto, Japan. He received his Ph.D. in Chemistry at Kwansei Gakuin University in 1990. He worked as JSPS fellow during 1990-1991 in Kwansei Gakuin University, research associate during 1991-1997 in Osaka City University (Department of Applied Physics), and associate professor during 1997-2002 in Shizuoka University (Department of Materials Engineering). During this last period he has experienced visiting associate professorships both in University of Tokyo (Physics Department) and University of Glasgow, Scotland, UK (Institute for Life Science). He moved to Osaka City University (Department of Physics) as a full professor in 2002. Throughout his academic career he has been focusing on the research of the primary process of photosynthesis. His research interest is now expanding to the regime of artificial photosynthesis. He has received several awards including BBSRC Japan-UK partnering award and SPACC-CSJ award. Since 2010 he has been the project leader of The OCU Advanced Research Institute for Natural Science and Technology (OCARINA) and now retired with emeritus professorship from Osaka City University in 2015. From 2015 he moved back to Kwansei Gakuin University as a full professor at the Department of Applied Chemistry for Environment, School of Science and Technology. 


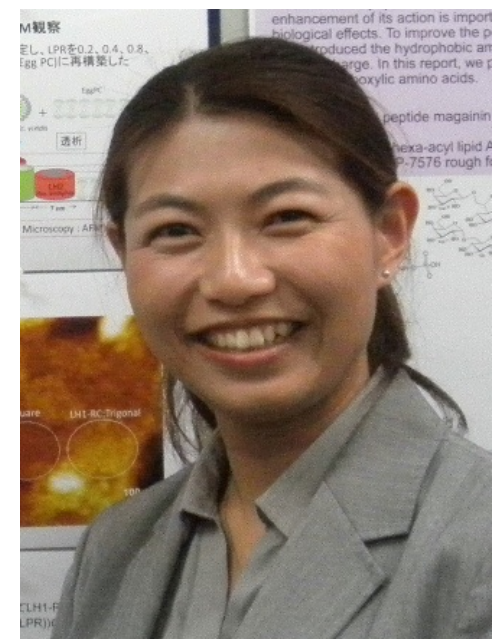

Yuko Sugai received her Ph.D degree in Chemistry at Nara Women's University, Japan in 2006. Since 2008 she joined Prof. Hashimoto's research group at Osaka City University, Japan and engaged in detecting the arrangement of light-harvesting pigment-protein complexes in reconstituted artificial photosynthetic membranes. Her current research interest is to create new functional light-harvesting materials using photosynthetic units. From 2015 she moved to Kwansei Gakuin University as a researcher, then she pursues to develop the new light-harvesting materials and to elucidate the functions under the direction of Prof. Hideki Hashimoto.

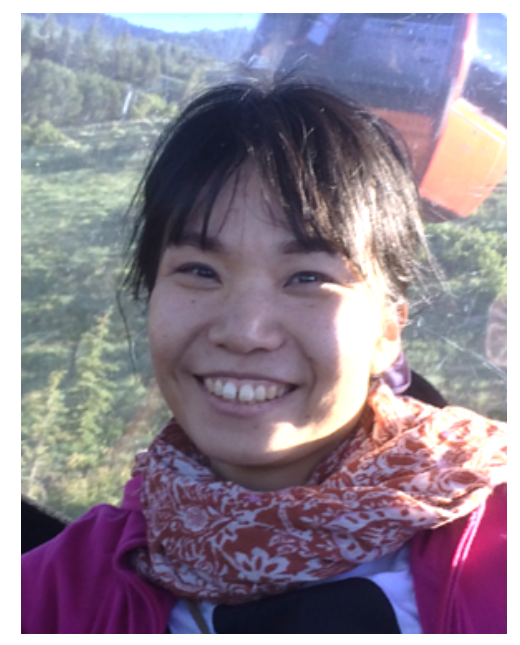

Chiasa Uragami was born in 1986 in Osaka, Japan. She received her Ph.D. in Physics at Osaka City University in 2015. She worked as JSPS Research fellow DC2 during 2013-2015 in Osaka City University and from 2015 to now, she works as a researcher in Kwansei Gakuin University (School of Science and Technology). Throughout her academic career she has been focusing on the research of the confocal Raman micro-spectroscopy of in vivo carotenoids. She measured the Raman spectra of the carotenoids penetrated to the rat skin and observed how fast the carotenoid molecule goes to the inner skin. This result was the first time in the world to determine the penetration rate of the carotenoids to the skin and it gave an impact to the beauty industry. She now has an interest on investigating the interaction between the carotenoids and the surrounding molecules using the Raman spectroscopy. 


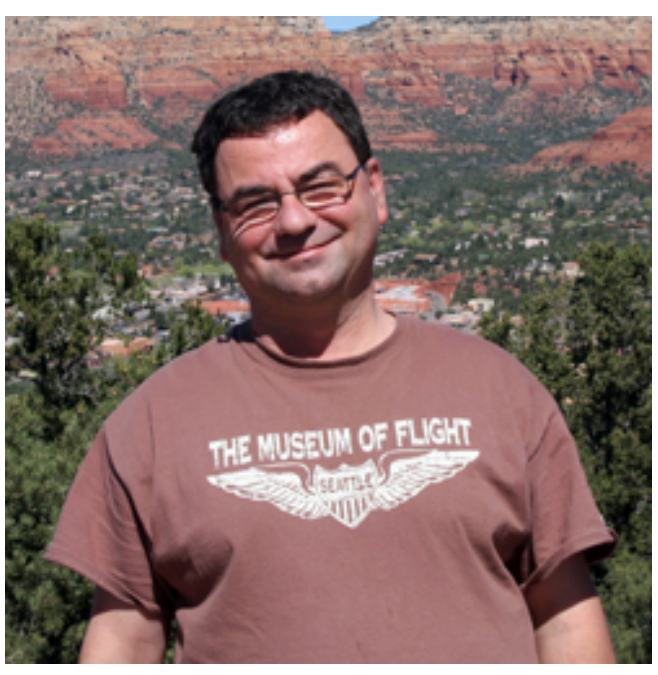

Alastair Thomas Gardiner was born in Hong Kong in 1964. He is a biochemist who obtained his Ph.D. from the University of Glasgow in 1992, after which he was awarded an Alexander von Humboldt Fellowship to join the group of Prof. Dr. W. Lubitz at the Max-Volmer-Institute for Biophysical Chemistry, at the Technical University Berlin. This time allowed him to become familiar with the application of a wide range of sophisticated magnetic resonance techniques such as, ENDOR, ESEEM and spin-polarised EPR, to photosynthetic complexes. Alastair subsequently obtained an EU TMR Fellowship to work at the Max-Planck-Institute for Biophysics, Frankfurt am Main, on purple photosynthetic membrane protein complex crystallisation. Since 1999 he has been a research assistant in the Institute of Molecular, Cellular and Systems Biology at the University of Glasgow.

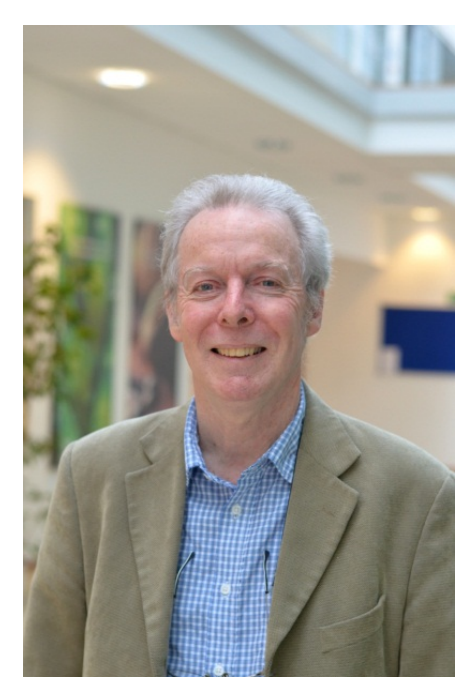

Richard Cogdell is the Hooker Professor of Botany at University of Glasgow and he is the Deputy Head there of the College of Medical, Veterinary and Life Sciences. He has been at Glasgow University since 1975 . He is a Fellow of the Royal Society and the Royal Society of Edinburgh. Currently he is President of the International Society for Photosynthesis Research and is on the Council of BBSRC. He was trained as a biochemist and most of his research has aimed at trying to understand the structure and function of the pigment protein complexes that carry out the very early light reactions of photosynthesis. 
Table 1. Carotenoid composition of some of the different RCs and light-harvesting complexes.

\begin{tabular}{|c|c|c|c|}
\hline \multicolumn{4}{|c|}{ Antenna Systems from Prokaryotes } \\
\hline Name & Type of RC & Oxygenic or Anoxygenic & Carotenoids \\
\hline Heliobacteria & Type I (FeS) & Anoxygenic & Diaponeurosporene \\
\hline $\begin{array}{l}\text { Green Sulfur } \\
\text { Bacteria }\end{array}$ & Type I (FeS) & Anoxygenic & Isorenieratene \\
\hline Purple Bacteria & $\begin{array}{l}\text { Type II } \\
\text { (Quinone) }\end{array}$ & Anoxygenic & $\begin{array}{l}\text { Spheroidene, } \\
\text { Spirilloxanthin, etc. }\end{array}$ \\
\hline $\begin{array}{l}\text { Filamentous } \\
\text { Green Bacteria }\end{array}$ & $\begin{array}{l}\text { Type II } \\
\text { (Quinone) }\end{array}$ & Anoxygenic & $\gamma$-carotene, $\beta$-carotene \\
\hline Cyanobacteria & $\begin{array}{l}\text { PS I (FeS) / } \\
\text { PS II (Quinone) }\end{array}$ & Oxygenic & $\beta$-carotene, Zeaxanthin \\
\hline \multicolumn{4}{|c|}{ Antenna Systems from Eukaryotes } \\
\hline Name & & Carotenoids & \\
\hline Chlorophytes, G & reen Algae & $\beta$-carotene, Lutein, Neo & anthin, Violaxanthin \\
\hline Red Algae & & Zeaxanthin & \\
\hline Dinoflagellates & & Peridinin & \\
\hline Cryptophytes & & $\alpha$-carotene, Alloxanthin & \\
\hline Heterokontophy & ta, Haptophyta & Fucoxanthin & \\
\hline
\end{tabular}




\section{Graphical Abstract}

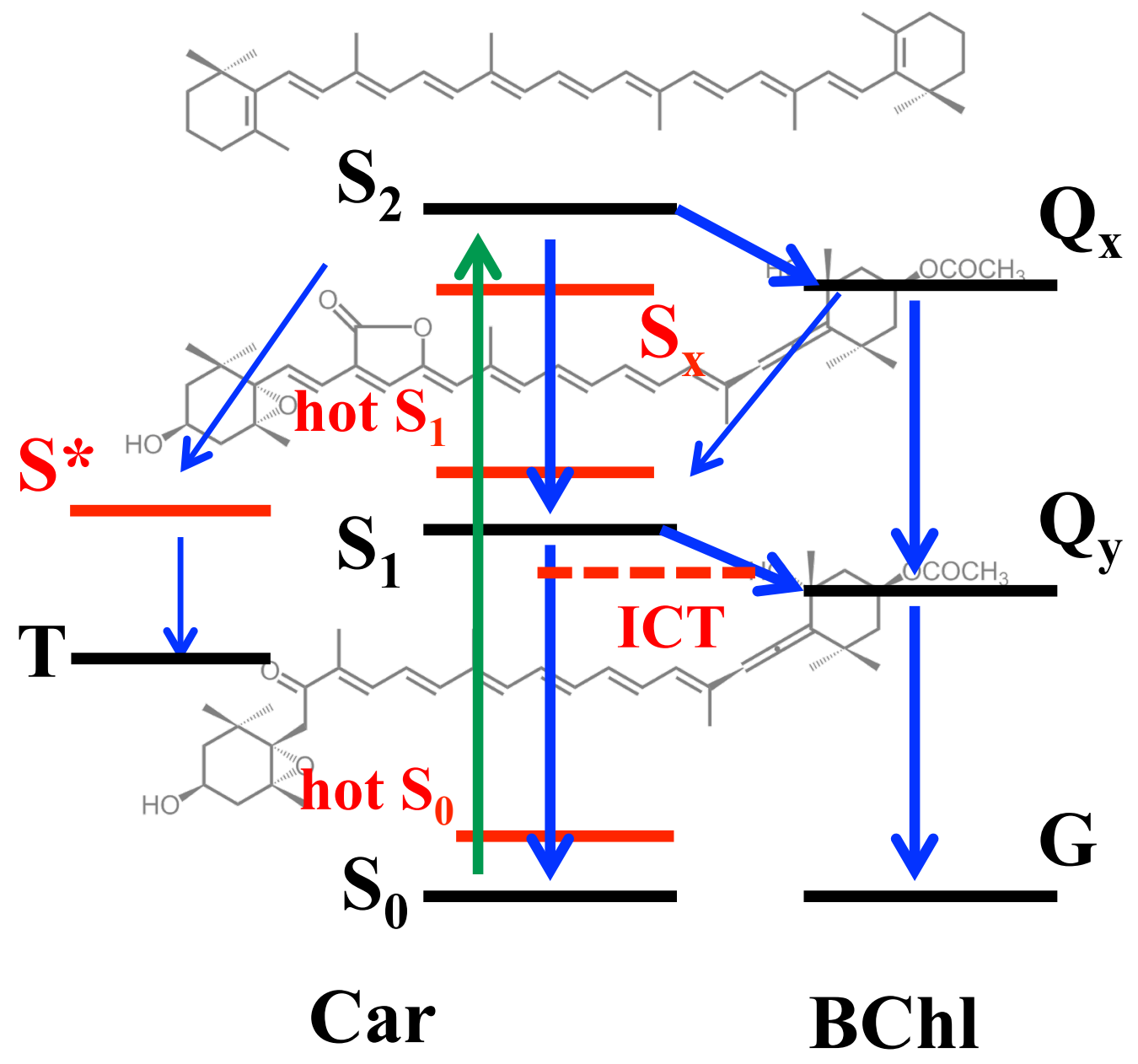

